\title{
Advances in Biomass Co-Combustion with Fossil Fuels in the European Context: A Review
}

\author{
Miroslav Variny $\left.{ }^{1, *} \mathbb{(}\right)$, Augustín Varga ${ }^{2}$, Miroslav Rimár ${ }^{3}$, Ján Janošovský ${ }^{1}$, Ján Kizek ${ }^{3}{ }^{\circledR}$, Ladislav Lukáč ${ }^{2}$, \\ Gustáv Jablonský ${ }^{2}$ il and Otto Mierka ${ }^{1}$ \\ 1 Department of Chemical and Biochemical Engineering, Faculty of Chemical and Food Technology, \\ Slovak University of Technology in Bratislava, Radlinského 9, 81237 Bratislava, Slovakia; \\ jan.janosovsky@stuba.sk (J.J.); otto.mierka@stuba.sk (O.M.) \\ 2 Department of Thermal Technology and Gas Industry, Institute of Metallurgy, Faculty of Materials, \\ Metallurgy and Recycling, Technical University of Kosice, Letná 9, 04200 Košice, Slovakia; \\ augustin.varga@tuke.sk (A.V.); ladislav.lukac@tuke.sk (L.L.); gustav.jablonsky@tuke.sk (G.J.) \\ 3 Department of Process Technique, Faculty of Manufacturing Technologies of the TU of Kosice with a Seat in \\ Prešov, Technical University of Kosice, Štúrova 31, 08001 Prešov, Slovakia; miroslav.rimar@tuke.sk (M.R.); \\ jan.kizek@tuke.sk (J.K.) \\ * Correspondence: miroslav.variny@stuba.sk; Tel.: +421-910-966-199
}

Citation: Variny, M.; Varga, A.; Rimár M.; Janošovský, J.; Kizek, J.; Lukáč, L.; Jablonský, G.; Mierka, O. Advances in Biomass Co-Combustion with Fossil Fuels in the European Context: A Review. Processes 2021, 9, 100 https://doi.org/10.3390/pr9010100

Received: 18 December 2020 Accepted: 31 December 2020 Published: 5 January 2021

Publisher's Note: MDPI stays neutral with regard to jurisdictional clai$\mathrm{ms}$ in published maps and institutional affiliations.

Copyright: $@ 2021$ by the authors. Licensee MDPI, Basel, Switzerland. This article is an open access article distributed under the terms and conditions of the Creative Commons Attribution (CC BY) license (https:// creativecommons.org/licenses/by/ $4.0 /)$.

\begin{abstract}
Co-combustion of biomass-based fuels and fossil fuels in power plant boilers, utility boilers, and process furnaces is a widely acknowledged means of efficient heat and power production, offering higher power production than comparable systems with sole biomass combustion. This, in combination with $\mathrm{CO}_{2}$ and other greenhouse gases abatement and low specific cost of system retrofit to co-combustion, counts among the tangible advantages of co-combustion application. Technical and operational issues regarding the accelerated fouling, slagging, and corrosion risk, as well as optimal combustion air distribution impact on produced greenhouse gases emissions and ash properties, belong to intensely researched topics nowadays in parallel with the combustion aggregates design optimization, the advanced feed pretreatment techniques, and the co-combustion life cycle assessment. This review addresses the said topics in a systematic manner, starting with feed availability, its pretreatment, fuel properties and combustor types, followed by operational issues, greenhouse gases, and other harmful emissions trends, as well as ash properties and utilization. The body of relevant literature sources is table-wise classified according to numerous criteria pertaining to individual paper sections, providing a concise and complex insight into the research methods, analyzed systems, and obtained results. Recent advances achieved in individual studies and the discovered synergies between co-combusted fuels types and their shares in blended fuel are summed up and discussed. Actual research challenges and prospects are briefly touched on as well.
\end{abstract}

Keywords: fossil fuel; co-combustion; greenhouse gases; ash; synergy; biomass pretreatment; reburning; oxy-combustion

\section{Introduction}

With increased awareness of climate change and the ongoing effort to reduce greenhouse gas emissions, solutions are sought for replacement of fossil fuels by renewable ones $[1,2]$. Biomass and various wastes are perceived as promising materials to produce energy, fuels, and chemicals [3-6]. Technologies facilitating production of electric energy in a more efficient way compared to biomass- and waste-based steam and power plants [7] include co-combustion in fossil fuel power plants [8,9], gasification, and pyrolysis [10-12]. While the latter two still represent a substantial challenge regarding the feed properties $[13,14]$, key equipment design, and operation, co-firing offers a less costly and simpler option for fossil fuel consumption reduction in the heat and power production sector $[15,16]$.

Biomass and waste co-combustion has been intensely researched in the last decade. Pilot and full scale systems studies conducted several decades ago [17-19] continue to 
be published [20,21], but they are more recently accompanied by laboratory experiments employing drop tube furnaces [22-24], thermogravimetric analyses (TGA) [25-27], and other laboratory equipment [28,29]. Experience with co-combustion systems operation are reported in the USA [30,31], Europe [32], as well as other parts of the world [33], generally claiming reduction in $\mathrm{CO}_{2}$ and other greenhouse gases emissions [34] with only minor changes in co-fired power plants and utility boilers thermal efficiencies [35]. Operational issues including the propensity for boiler slagging [36], fouling, and corrosion [15] are still not tackled sufficiently, though. Improved understanding of these aspects [37-40] aids the assessment of co-combustion sustainability. Greenhouse gases (GHG) emissions [41-43] and other emission $[44,45]$ trends with varying biomass type and share and process conditions are studied and evaluated. New biomass and waste types are studied [46-48], searching for optimal co-combustion conditions, exploring material synergies [49] and improved properties and utilization of produced ash $[50,51]$. Feed storage and pretreatment [52] is another important factor influencing the co-combustion economics. Despite these challenges, co-combustion of biomass, combustible industrial byproducts, and wastes is acknowledged as a feasible part of the near- and mid-term environmental strategy of the European Union (EU) [2,53]. Performed techno-economic studies [54-56] contribute to the co-combustion applicability assessment. The modeling and computational approach to various scale systems performance is more widespread [57-59], which contributes to the understanding of co-combustion systems' behavior and interactions.

These trends document the need for a review of the biomass and waste co-combustion technology state of the art, recent advances therein, and the challenges it faces. Paper organization is as follows: First, biomass availability is assessed, followed by a survey on biomass and fossil fuel basic properties. Next, relevant biomass pretreatment techniques are discussed. Alternative co-combustion technologies are briefly presented, and a separate part is dedicated to co-combustion equipment. Parts dealing with combustion products properties (ash, flue gases) and the related fouling, slagging, and corrosion issues as well as with harmful emissions and process modification possibilities for their alleviation follow. Future challenges are debated briefly, and a short Conclusions part ends the review.

\section{Biomass and Waste Availability, Co-Combustion Technical Potential, Biomass Supply Chain, and $\mathrm{CO}_{2} \mathrm{LCA}$}

A review on biomass co-combustion in coal power plants in the EU by Hansson et al. (2009) [9] identified its technical potential as 520 to $940 \mathrm{PJ} /$ year depending on the coal boilers age, below 40 or below 30 years, considered for co-combustion. This biomass potential in conversion to power production represented less than $5 \%$ of gross national electricity production in most EU countries. Technical potential increase of around $25 \%$ to $40 \%$ can be expected with the facilities under construction or planned to be built in the near future. With the prospect of old coal power plants shutdown, the co-combustion potential decreased on the other hand, and it should be close to zero in 2040. Overall potential of residual and waste biomass in the EU-27 for the use in energy production sector exceeded $6000 \mathrm{PJ} /$ year as concluded by both Hansson et al. (2009) [9] and Alakangas et al. (2012) [60], with woody residues, firewood, and herbaceous and fruit biomass representing more than two thirds of the total biomass [60]. More recent studies estimated this potential as high as 11,500 PJ/year in the EU-28 + Western Balkan countries [61], while solely in Germany, the annually available unused biomass amounted up to 30 to 47 million tons [62], representing roughly 250 to $400 \mathrm{PJ} /$ year of energy content.

McIlveen-Wright et al. (2011) [54] simulated a range of power plants by the European Coal Liquefaction Process Simulation and Evaluation (ECLIPSE) simulation software package in different co-combustion configurations to estimate and compare their breakeven electricity selling price (BESP), with a $600 \mathrm{MW}_{\mathrm{e}}$ supercritical pulverized coal-fired power plant with flue gas desulfurization plant serving as a benchmark. Due to considered fuel prices, all co-combustion options suffered from higher BESP; its difference amounted to around $10 \$ / \mathrm{MWh}$ for large scale $\left(\geq 250 \mathrm{MW}_{\mathrm{e}}\right)$ systems with $20 \%$ biomass share but exceeded $50 \$ / \mathrm{MWh}$ for small scale $\left(\leq 25 \mathrm{MW}_{\mathrm{e}}\right)$ systems with over $50 \%$ biomass share. The 
$\mathrm{CO}_{2}$ credit needed to reach the same BESP ranges from 50 to $500 \$ / \mathrm{t} \mathrm{CO}$, depending on system size and biomass share.

Agbor et al. (2016) [55] studied 60 biomass co-combustion scenarios with coal and natural gas in pulverized coal boilers and natural gas (NG) fired combined cycles (CC). A detailed economic analysis was presented yielding incremental and levelized electricity cost. Both parameters increased with the increasing biomass share. Levelized electricity cost ranged between 55 and 75 \$/MWh, which was by 5 to 25 \$/MWh more than the reference electricity cost in 2014. Parallel to this, avoided $\mathrm{CO}_{2}$ emission cost was calculated, amounting to 70 to $370 \$ / \mathrm{tCO}_{2}$ and decreasing with the increasing biomass share, which is substantially lower for the NGCC based scenarios compared to coal boiler-based ones.

López et al. (2018) [63] performed a techno-economic analysis of variable biomass share and plant electric output oxycombustion supercritical power plant and evaluated cost of energy, cost of avoided $\mathrm{CO}_{2}$ emissions, and net present value (NPV) for individual scenarios. The obtained results indicate that plant size affects the values of these performance parameters more than the biomass share, and an economically acceptable solution can be found in combination of plant size increase and biomass co-combustion.

Miedema et al. (2017) [56] compared various biomass supply chains with the coal one in Dutch conditions considering energy efficiency, renewable energy production, and GHG emissions. Each operation (mining/harvesting, transport, pretreatment) in the supply chain was attributed an interval of energy consumption and $\mathrm{CO}_{2}$ release. Torrefaction, chipping, pelletization, and combination of pelletization and torrefaction were considered as biomass pretreatment options. Calculations yielded that for $60 \%$ biomass energy share, total energy consumption increases by up to 30\%, and GHG emissions decrease by up to $50 \%$. The combined torrefaction and pelletization scenario exhibited best results. The authors concluded that the increase of total energy consumption with the increasing biomass share indicates that biomass introduction in power generation does not necessarily lead to a more efficient system, and under certain circumstances the GHG emission decrease can be even negligible.

Various other studies dealt with multifaceted aspects of biomass or waste co-combustion in terms of environmental, economic, or energetic performance within national or regional frames. An overview of these is provided in Table 1.

Relevant literature overviewed in Table 1 documents the actual trend of analyzing and modeling co-combustion utilization potential by various techniques and assessing its related secondary spatio-temporal aspects on the industry, environment, and society.

Table 1. References to relevant papers covering various co-combustion aspects. Legend: EU = European Union, $\mathrm{LCA}=$ life cycle assessment, $\mathrm{GHG}=$ greenhouse gases emissions, GWP = global warming potential, NPV = net present value, ECLIPSE = European Coal Liquefaction Process Simulation and Evaluation simulation software package, FB = fluidized bed, $\mathrm{CC}=$ carbon capture, $\mathrm{PI}=$ performance indicator, TGA = thermogravimetric analysis, $\mathrm{TIC}=$ total investment cost.

\begin{tabular}{|c|c|c|c|}
\hline Reference & Studied Systems & Approach & Evaluated Aspects \\
\hline Hein and Bemtgen (1998) [17] & $\begin{array}{l}\text { Various fuels, co-feeding } \\
\text { ratios, boiler types, and } \\
\text { thermal outputs from } 0.3 \text { to } \\
\qquad 150 \mathrm{MW}_{\mathrm{t}}\end{array}$ & EU project; Experimental & $\begin{array}{l}\text { Fuel conversion, Chlorine and } \\
\text { GHG emissions, content of } \\
\text { heavy metals in ash }\end{array}$ \\
\hline Hughes (2000) [64] & $\begin{array}{c}\text { Survey on realized } \\
\text { co-combustion application in } \\
\text { the USA }\end{array}$ & \multicolumn{2}{|c|}{$\begin{array}{l}\text { Opinion on economic, environmental, and political aspects of } \\
\text { co-combustion }\end{array}$} \\
\hline Mann and Spath (2001) [65] & $\begin{array}{l}\text { Coal fired power plant } 360 \\
\mathrm{MW}_{\mathrm{e}} \text { in co-combustion mode }\end{array}$ & Software analysis, LCA & $\begin{array}{l}\text { Gaseous and other emissions, } \\
\text { energy efficiency, biomass } \\
\text { utilization sensitivity to GWP }\end{array}$ \\
\hline Sami et al. (2001) [8] & $\begin{array}{l}\text { Detailed survey on realized } \\
\text { and planned co-combustion } \\
\text { application in the USA and in } \\
\text { the EU }\end{array}$ & Review paper & $\begin{array}{l}\text { Technical aspect: fuel } \\
\text { properties and preparation, } \\
\text { boiler types, emissions, } \\
\text { carbon burnout }\end{array}$ \\
\hline
\end{tabular}


Table 1. Cont.

\begin{tabular}{|c|c|c|c|}
\hline Reference & Studied Systems & Approach & Evaluated Aspects \\
\hline Royo et al. (2012) [66] & $\begin{array}{l}\text { Case of Spain, co-combustion } \\
\text { potential in existing coal fired } \\
\text { power plants }\end{array}$ & $\begin{array}{c}\text { Available biomass LCA } \\
\text { assessment }\end{array}$ & $\begin{array}{l}\text { Nation-wide GHG emissions } \\
\text { reduction and power from } \\
\text { biomass production potential }\end{array}$ \\
\hline $\begin{array}{l}\text { Lüschen and Madlener } \\
\qquad \text { (2013) [67] }\end{array}$ & $\begin{array}{c}\text { Case of Germany, } \\
\text { co-combustion potential in } \\
\text { new coal fired power plants }\end{array}$ & $\begin{array}{l}\text { Techno-economic analysis by } \\
\text { simulation. Marginal analysis. } \\
\text { Statistical evaluation. }\end{array}$ & $\begin{array}{l}\text { NPV, its sensitivity in various } \\
\text { scenarios. }\end{array}$ \\
\hline Gu et al. (2013) [68] & $\begin{array}{l}\text { Industrial FB boiler in } \\
\text { coal-biomass co-combustion } \\
\text { mode }\end{array}$ & Simulation software ECLIPSE & $\begin{array}{c}\text { Plant efficiency, } \mathrm{CO}_{2} \text { savings, } \\
\text { project NPV }\end{array}$ \\
\hline Kazagic et al. (2016) [69] & Coal power plant in Serbia & $\begin{array}{c}\text { Biomass availability } \\
\text { assessment, economic analysis }\end{array}$ & $\begin{array}{c}\text { Environmental and economic } \\
\text { PI }\end{array}$ \\
\hline Mohd Idris et al. (2018) [70] & $\begin{array}{l}\text { Power plant in Malaysia, coal } \\
\quad+\text { palm oil biomass }\end{array}$ & $\begin{array}{l}\text { Biomass supply chain } \\
\text { assessment, techno-economic } \\
\text { assessment and optimization }\end{array}$ & $\begin{array}{l}\text { Multicriterial optimization of } \\
\text { biomass utilization, } \\
\text { environmental and economic } \\
\text { PI }\end{array}$ \\
\hline $\begin{array}{l}\text { Nyashina and Strizhak } \\
\text { (2018) [71] }\end{array}$ & $\begin{array}{c}\text { Potential of composite } \\
\text { fossil—biomass fuel use in } \\
\text { Russia }\end{array}$ & $\begin{array}{l}\text { Laboratory rotary muffle } \\
\text { furnace + gas analyzer }\end{array}$ & Environmental PI \\
\hline Pikoń et al. (2019) [72] & $\begin{array}{l}\text { Comparative analysis, waste } \\
\text { fuel and lignite_-case of } \\
\text { Poland }\end{array}$ & $\begin{array}{c}\text { Laboratory TGA, LCA based } \\
\text { on the results }\end{array}$ & Environmental PI \\
\hline Smolinski et al. (2019) [73] & $\begin{array}{c}\text { Fuel produced from sewage } \\
\text { sludge treatment and } \\
\text { coal—case of Poland }\end{array}$ & $\begin{array}{c}\text { Pilot plant for sewage sludge } \\
\text { treatment; profitability } \\
\text { analysis }\end{array}$ & $\begin{array}{c}\text { Economic and environmental } \\
\text { PI }\end{array}$ \\
\hline Truong et al. (2019) [74] & $\begin{array}{l}\text { Assessment of straw and rice } \\
\text { husk cofiring in Vietnam }\end{array}$ & $\begin{array}{l}\text { Spatio-temporal analysis, } \\
\text { techno-economic evaluation, } \\
\text { sensitivity analysis }\end{array}$ & $\begin{array}{l}\text { GHG emissions reduction, } \\
\text { fossil, and renewable } \\
\text { electricity production costs }\end{array}$ \\
\hline Glushkov et al. (2020) [48] & \multicolumn{2}{|c|}{$\begin{array}{l}\text { Testing co-combustion of new composite fuels in a laboratory } \\
\text { tube muffle furnace, results applied to three regions in Russia } \\
\text { via fossil fuel saving calculations. }\end{array}$} & TIC, financial savings \\
\hline
\end{tabular}

\section{Biomass and Fossil Fuel Properties}

Fuel composition resulting from its proximate and ultimate analysis as well as lower heating value counts among the main fuel quality parameters [8]. Table 2 presents characterization of a sample of biomass sources potentially available for co-firing, whereas Table 3 gives an overview of some solid fossil fuels characteristics. Biomass and waste generally contain more volatile matter and less fixed carbon and ash than coal, which in turn impacts their combustion characteristics.

As it results from Tables 2 and 3, biomass, waste materials, and other solid materials that can be considered for co-combustion usually have much lower share of fixed carbon and much higher share of volatiles that common coals. Combustion of such solids proceeds faster, posing a problem in grate combustors if fuel is not well premixed, especially with bulk solid fuels like chips or package material. After a quick burnout of such unevenly distributed materials, locations with little or no fuel can form on the grate, which can subsequently lead to uneven combustion air distribution and, eventually, to grate damage. Fuel pretreatment via pelletization or torrefaction can increase the density and improve the burnout characteristics of bulk fuels, but it requires additional investment and operation costs [10].

Moisture content is an additional factor influencing fuel burnout. Elevated moisture content can be found in waste fuels such as woody residues or generally in fuels stored in open spaces. Apart from directly impacting the boiler and plant efficiency, it can contribute 
to higher greenhouse gases emissions due to its worsened burnout. Fuel pretreatment is dealt with in Section 4.

Table 2. Overview of basic properties of some biomass and waste materials. Legend: ad = air dry basis, $\mathrm{Al}=\mathrm{aluminum}$, $\mathrm{ar}=$ as received basis, $\mathrm{daf}=\mathrm{dry}$ ash free basis, $\mathrm{db}=$ dry basis, $\mathrm{LHV}=$ lower heating value, $\mathrm{RDF}=$ refuse derived fuel.

\begin{tabular}{|c|c|c|c|c|c|c|c|c|c|}
\hline Reference & [75] & [75] & [76] & [76] & [77] & [78] & [27] & [79] & [80] \\
\hline Sample & Straw & Wood & $\begin{array}{c}\text { Eucalyptus } \\
\text { Bark }\end{array}$ & $\begin{array}{l}\text { Rice } \\
\text { Husk }\end{array}$ & $\begin{array}{l}\text { Woody } \\
\text { Residues }\end{array}$ & $\begin{array}{c}\text { Laying } \\
\text { Hens } \\
\text { Manure }\end{array}$ & $\begin{array}{c}\text { RDF } \\
\text { (Sample } \\
\text { Plastics } \\
\text { Blend) }\end{array}$ & $\begin{array}{c}\text { Automobile } \\
\text { Shredder } \\
\text { Residue }\end{array}$ & $\begin{array}{c}\text { Aseptic Milk } \\
\text { Package } \\
\text { (Tetrapak) }\end{array}$ \\
\hline \multicolumn{10}{|c|}{ Proximate analysis, \% wt. } \\
\hline $\begin{array}{l}\text { Fixed } \\
\text { carbon }\end{array}$ & 15.44 & 17.05 & 39.9 & 18.6 & 9.3 & $3.67 \mathrm{db}$ & $6.07 \mathrm{db}$ & 10.00 & 8.16 \\
\hline $\begin{array}{l}\text { Volatile } \\
\text { matter }\end{array}$ & 66.40 & 74.14 & 43.1 & 54.4 & 37.4 & $67.01 \mathrm{db}$ & $82.67 \mathrm{db}$ & 71.10 & 76.92 \\
\hline Ash & 7.13 & 0.41 & 10.7 & 18.2 & 0.4 & $29.32 \mathrm{db}$ & $11.25 \mathrm{db}$ & 18.00 & $5.96+6.1 \mathrm{Al}$ foil \\
\hline Moisture & 11.03 & 8.40 & 6.3 & 8.8 & 52.9 & 12.17 & 1.23 & 0.50 & 2.8 \\
\hline \multicolumn{10}{|c|}{ Ultimate analysis, \% wt. } \\
\hline Carbon & 38.88 ar & 45.94 ar & 41.70 ar & 37.47 & $52.4 \mathrm{db}$ & $38.40 \mathrm{db}$ & $58.09 \mathrm{db}$ & 58.88 daf & 47.33 daf \\
\hline Hydrogen & 5.44 ar & 5.50 ar & 4.60 ar & 4.64 & $5.9 \mathrm{db}$ & $5.91 \mathrm{db}$ & $9.38 \mathrm{db}$ & 6.75 daf & $7.17 \mathrm{daf}$ \\
\hline Oxygen & 36.75 ar & 39.65 ar & 36.10 ar & 30.90 & $40.6 \mathrm{db}$ & 21.74 & $20.39 \mathrm{db}$ & 13.55 daf & 30.26 daf \\
\hline Nitrogen & 0.70 ar & 0.08 ar & 0.54 ar & 0.59 & $0.19 \mathrm{db}$ & 4.19 & $0.35 \mathrm{db}$ & $1.42 \mathrm{daf}$ & $0.26 \mathrm{daf}$ \\
\hline Sulfur & 0.01 ar & 0.02 ar & 0.06 ar & - & $0.022 \mathrm{db}$ & 0.44 & - & 0.90 daf & $0.06 \mathrm{daf}$ \\
\hline $\begin{array}{c}\mathrm{LHV} \\
\mathrm{MJ} / \mathrm{kg}\end{array}$ & 14.35 ar & 17.13 ar & 14.70 ar & 14.36 ar & 9.924 ar & $12.36 \mathrm{ad}$ & $31.23 \mathrm{db}$ & - & - \\
\hline
\end{tabular}

Table 3. Overview of basic properties of some solid fossil fuels.

\begin{tabular}{|c|c|c|c|c|c|c|c|c|}
\hline Reference & [81] & [81] & [15] & [82] & [83] & [46] & [84] & [85] \\
\hline Sample & $\begin{array}{l}\text { Highvale } \\
\text { Coal }\end{array}$ & $\begin{array}{c}\text { Eastern } \\
\text { Bitumi- } \\
\text { nous }\end{array}$ & $\begin{array}{l}\text { Illinois } \\
\text { Bitumi- } \\
\text { nous }\end{array}$ & $\begin{array}{l}\text { Czech Coal } \\
\text { "Sokolov" }\end{array}$ & $\begin{array}{c}\text { Yunnan } \\
\text { Low } \\
\text { Rank } \\
\text { Coal }\end{array}$ & $\begin{array}{c}\text { Industrial } \\
\text { Coal } \\
\text { Slurry }\end{array}$ & $\begin{array}{l}\text { Taldinsky } \\
\text { Hard Coal }\end{array}$ & $\begin{array}{c}\text { B- } \\
\text { Goynuk } \\
\text { Lignite }\end{array}$ \\
\hline \multicolumn{9}{|c|}{ Proximate analysis, $\%$ wt. } \\
\hline Fixed carbon & 43.89 & 55.52 & 44.2 & 33.64 & $31.91 \mathrm{db}$ & $36.62 \mathrm{ad}$ & 46.28 & 23.65 \\
\hline Volatile matter & 37.15 & 34.91 & 37.4 & 37.19 & $21.40 \mathrm{ab}$ & $57.51 \mathrm{ad}$ & 32.92 & $39.63 \mathrm{db}$ \\
\hline Ash & 11.39 & 8.82 & 14.7 & 22.46 & $46.70 \mathrm{db}$ & $34.92 \mathrm{ad}$ & 8.67 & $24.59 \mathrm{db}$ \\
\hline Moisture & 7.61 & 0.75 & 3.7 & 6.71 & 7.0 & $0.95 \mathrm{ad}$ & 12.13 & 33.91 \\
\hline \multicolumn{9}{|c|}{ Ultimate analysis, \% wt. } \\
\hline Carbon & $60.70 \mathrm{db}$ & $77.33 \mathrm{db}$ & $80.2 \mathrm{daf}$ & $50.20 \mathrm{daf}$ & $40.44 \mathrm{db}$ & $53.29 \mathrm{ad}$ & 63.45 ar & $55.57 \mathrm{db}$ \\
\hline Hydrogen & $4.01 \mathrm{db}$ & $5.08 \mathrm{db}$ & 5.7 daf & $4.01 \mathrm{daf}$ & $2.99 \mathrm{db}$ & $3.89 \mathrm{ad}$ & 4.79 ar & $10.29 \mathrm{db}$ \\
\hline Oxygen & $21.86 \mathrm{db}$ & $6.29 \mathrm{db}$ & $7.0 \mathrm{daf}$ & $15.49 \mathrm{daf}$ & $7.05 \mathrm{db}$ & $9.41 \mathrm{ad}$ & $8.85 \mathrm{ar}$ & $4.93 \mathrm{db}$ \\
\hline Nitrogen & $0.84 \mathrm{db}$ & $1.45 \mathrm{db}$ & $1.7 \mathrm{daf}$ & $0.73 \mathrm{daf}$ & $0.78 \mathrm{db}$ & $0.83 \mathrm{ad}$ & $1.81 \mathrm{ar}$ & $1.73 \mathrm{db}$ \\
\hline Sulfur & $0.28 \mathrm{db}$ & $0.96 \mathrm{db}$ & $5.4 \mathrm{daf}$ & $0.58 \mathrm{daf}$ & $2.05 \mathrm{db}$ & $0.65 \mathrm{ad}$ & $0.30 \mathrm{ar}$ & $2.89 \mathrm{db}$ \\
\hline $\begin{array}{l}\text { Higher heating } \\
\text { value, } \mathrm{MJ} / \mathrm{kg}\end{array}$ & 21.59 ar & 32.13 ar & 27.31 ar & $19.56(\mathrm{LHV}, \mathrm{ar})$ & $14.46 \mathrm{ad}$ & $22.07 \mathrm{ad}$ & $25.02(\mathrm{LHV}, \mathrm{ar})$ & $20.62 \mathrm{db}$ \\
\hline
\end{tabular}

Table 4 provides an overview of basic ash chemical composition of selected materials. The presence and the ratio of basic $(\mathrm{Fe}, \mathrm{Ca}, \mathrm{Mg}, \mathrm{Na}, \mathrm{K}, \mathrm{P})$ and acidic $(\mathrm{Si}, \mathrm{Al}, \mathrm{Ti})$ oxides content in ash determine its fusion properties and thus, in turn, its fouling and slagging tendency. Pronobis (2006) [37] defined the base to acid ratio as a measure of such ash behavior, alternatively termed slagging index [86]. Lawrence et al. (2008) [87] defined the 
fouling index as a novel tool for slagging propensity quantification. The higher the values of both indices, the higher is the slagging and fouling tendency of the given ash. Biomass ash generally exhibits stronger slagging and fouling tendencies than coal ash, which is corroborated by the values of both indices listed in Table 4 . Most commonly used indices for slagging and fouling propensity expression are summed up in Sajdak et al. (2019) [88].

Table 4. Basic ash composition analysis of some biomass and waste materials and fossil fuels. Subbit. = Subbituminous.

\begin{tabular}{|c|c|c|c|c|c|c|c|c|c|c|}
\hline Reference & [18] & [38] & [89] & [90] & [91] & [92] & [15] & [38] & [89] & [91] \\
\hline $\begin{array}{c}\text { Sample } \\
\text { Compound \% wt. }\end{array}$ & $\begin{array}{l}\text { Wheat } \\
\text { Straw }\end{array}$ & $\begin{array}{l}\text { Sakura } \\
\text { Wood }\end{array}$ & $\begin{array}{c}\text { Red } \\
\text { Oak } \\
\text { Wood }\end{array}$ & $\begin{array}{l}\text { Soybean } \\
\text { Stalk }\end{array}$ & $\begin{array}{l}\text { Poultry } \\
\text { Litter }\end{array}$ & $\begin{array}{c}\text { Blend } \\
\text { of } \\
\text { Fibrous } \\
\text { and } \\
\text { Plastic } \\
\text { Waste }\end{array}$ & $\begin{array}{c}\text { US } \\
\text { Wyoming } \\
\text { Subbit. } \\
\text { Coal }\end{array}$ & $\begin{array}{l}\text { Bituminous } \\
\text { Coal }\end{array}$ & Coal & $\begin{array}{l}\text { Turkish } \\
\text { Lignite }\end{array}$ \\
\hline $\mathrm{SiO}_{2}$ & 58.0 & 5.6 & 49 & 33.18 & 4.73 & 33 & 48 & 64.2 & 42 & 53.71 \\
\hline $\mathrm{Al}_{2} \mathrm{O}_{3}$ & 3.2 & 1.4 & 9.5 & 2.55 & 1.09 & 27 & 19 & 21.4 & 20 & 24.08 \\
\hline $\mathrm{Fe}_{2} \mathrm{O}_{3}$ & 1.5 & 2.2 & 8.5 & 1.75 & - & 0.9 & 12 & 4.45 & 17 & - \\
\hline $\mathrm{CaO}$ & 6.0 & 51.7 & 17.5 & 30.95 & 39.52 & 21 & 16 & 1.02 & 5.5 & 2.48 \\
\hline $\mathrm{MgO}$ & 1.4 & 17.2 & 1.1 & 8.23 & 6.22 & 3.0 & 4.1 & 0.88 & 2.1 & 1.73 \\
\hline $\mathrm{Na}_{2} \mathrm{O}$ & 0.9 & 0.6 & 0.5 & 3.56 & 5.11 & 1.6 & 0.7 & 0.58 & 1.4 & 0.76 \\
\hline $\mathrm{K}_{2} \mathrm{O}$ & 14.0 & 4.4 & 9.5 & 15.72 & 26.53 & 0.6 & 1.0 & 1.52 & 5.8 & 1.41 \\
\hline $\mathrm{P}_{2} \mathrm{O}_{5}$ & 2.8 & 2.9 & 1.8 & 2.43 & - & - & - & 0.19 & - & - \\
\hline Slagging index [37] & 0.43 & 11.29 & 0.66 & 1.75 & 13.30 & 0.45 & 0.50 & 0.10 & 0.51 & 0.08 \\
\hline Fouling index [87] & 6.5 & 56.4 & 6.6 & 33.8 & 420.7 & 1.0 & 0.9 & 0.2 & 3.7 & 0.2 \\
\hline
\end{tabular}

Combining the information from Tables 2-4, it can be concluded that ash content of woody biomass is usually lower than that of coals, whereas that of herbaceous biomass, bark, and various solid wastes is comparable with that of most coals. However, slagging and fouling tendency of individual materials is influenced by a higher extent by the base elements in ash than by ash content in the material solely. High ash fuels with high base in ash content can be considered as the most problematic ones. Higher content of $\mathrm{SiO}_{2}$ and $\mathrm{Al}_{2} \mathrm{O}_{3}$ in common coal ash compared to biomass ashes may be beneficial, as it forms aluminosilicates with base elements trapping them effectively in ash in co-combustion applications [11,13].

\section{Biomass Pretreatment}

Biomass and waste exhibit several properties which impact their direct co-combustion feasibility:

- $\quad$ Low bulk energy density

- $\quad$ Higher moisture content

- Higher content of volatiles

Low energy density can be solved by decreasing the moisture content by pre-drying, pelletization, or their combination. Removing excess moisture by low potential heat application is a widely used unit operation $[93,94]$, often integrated in heat and power plants with exhaust flue gas or low-pressure steam used as a cheap heat source [52,95]. Biomass with lower moisture has higher calorific value, and it generally yields more stable flame and does not require a high-quality supplementary fuel (usually natural gas) for the combustion process stabilization in the combustion chamber [95]. Dried biomass or waste can further be processed by milling, pelletization, or torrefaction depending on the combustion equipment design [31]. Similarly, composite pellets can be produced to improve the combustion characteristics of various solid fuels, wastes, and their mixtures, even with liquid component addition [96]. While pre-drying or other feedstock pretreatment improves its heating value and combustion characteristics, it is not recommended under any circumstances. A techno-economic study must provide the necessary inputs 
for decision-making considering the benefits in form of expectable higher boiler thermal efficiency and improved fuel burnout along with extra capital and operational costs related to logistics, storage capacities, additional infrastructure, and heat/power input needed. The generally positive results from laboratory studies on feedstock pretreatment should therefore be evaluated conservatively before application in industrial-scale systems.

Heating of biomass and waste to higher temperatures than necessary for their drying results in the release of volatile compounds and a change in its macromolecular structure. Its pretreatment in the temperature range of 200 to $300{ }^{\circ} \mathrm{C}$ with exposure times of up to $2 \mathrm{~h}$ in inert atmosphere is termed torrefaction [97,98], and it reduces the $\mathrm{H} / \mathrm{C}$ and $\mathrm{O} / \mathrm{C}$ ratio of the processed material and, thus, shifts its combustion characteristics closer to that of solid fossil fuels. The calorific value, structure, and bulk density of pretreated feedstock are also affected [99]. Loss of volatiles by torrefaction must, however, be accounted for in the material, energy, and economic balance of its pretreatment, together with heat needed for feedstock drying and torrefaction [100,101].

$\mathrm{Xu}$ et al. (2018) [92] studied physical properties of various waste material blends after their torrefaction in a convective furnace at $300^{\circ} \mathrm{C}$ and exposure time of 3 to $120 \mathrm{~min}$. They concluded that, depending on the material type, its heating value remained the same or increased, and the material loss was up to 55\%. Tumuluru (2015) [102] assessed the changes in composition and energy properties of switchgrass and corn stover samples during torrefaction with varying temperature $\left(180\right.$ to $\left.270{ }^{\circ} \mathrm{C}\right)$ and residence time (15 to $120 \mathrm{~min}$ ) in a thermogravimetric analyzer. The obtained results indicated a marginal improvement in higher heating value (HHV) of the studied samples and a significant (up to 58\%) mass loss both due to moisture content reduction and extensive devolatilization at higher temperatures and longer exposures. The $\mathrm{H} / \mathrm{C}$ ratio decreased from initial values of over 1.4 to around 0.6 under the most severe torrefaction conditions, while the observed trend for the $\mathrm{O} / \mathrm{C}$ ratio was similar (decrease from 1.3 to 0.7 ). Similar results were achieved by torrefaction of other feedstock, including herbaceous, agricultural [99], and woody wastes [100].

Biomass based feedstock leaching has recently been intensely studied as a means of mineralogical composition adjustment to decrease its slagging and fouling propensity. Full scale results are still scarce. A comprehensive summary on this technique was provided by Madanayake et al. (2017) [103].

Table 5 sums up basic characteristics of the main body of analyzed papers. No biomass or waste pretreatment technique was used in the majority of studies, while drying and torrefaction are quite common techniques employed in laboratory scale studies. Various materials and their blends were studied nearly as often as single biomass or waste co-combustion studies. The studies are further classified and evaluated in Tables 6-10 showing individual system performance, plant layout, and operational issues aspects. See Table 6 below. 
Table 5. Basic characteristics of the main body of relevant papers. Legend: Ref.-reference; 1 -Paper type $(\mathrm{O}=$ original, $\mathrm{R}=$ review); 2-paper publication year; 3-feedstock (Fos. = Fossil, $\mathrm{B}+\mathrm{W}=$ Biomass + waste, $\mathrm{S}=$ single type, $\mathrm{M}=$ two or more types); 4 -Biomass /waste pretreatment $(\mathrm{D}=$ drying, $\mathrm{T}=$ torrefaction, $\mathrm{G}=$ gasification, $\mathrm{Ot}$. = other, $\mathrm{N}=$ no pretreatment, $\mathrm{V}=$ various methods); 5-System scale $(\mathrm{C}=$ modeling/calculation approach, $\mathrm{L}=$ laboratory, $p=$ pilot plant, FS = full scale, $\mathrm{M}=$ multiple systems and/or scales, $\mathrm{PPB}$ - power plant boiler, IB-industrial boiler, PFBA = pressurized fluidized bed apparatus, $\mathrm{PCB}=$ pulverized coal boiler, $\mathrm{EFR}=$ entrained flow rector, $\mathrm{BG}=$ biomass gasifier, $\mathrm{PY}=$ pyrolyzer, $\mathrm{REB}=$ reburning, $\mathrm{BF}=$ batch furnace, $\mathrm{DTF}=$ drop tube furnace, $\mathrm{IFR}=$ isothermal flow reactor, $\mathrm{CFBC}=$ circulating fluidized bed combustor, $\mathrm{F}=$ furnace, $\mathrm{PFF}=$ pulverized fuel furnace, $\mathrm{CB}=$ coal boiler, $\mathrm{PFC}=$ pulverized fuel combustor, $\mathrm{SPR}=$ single particle reactor, $\mathrm{TF}=$ tube furnace, $\mathrm{MG}=$ moving grate, $\mathrm{MIA}=$ multi-instrumental analysis, $\mathrm{UGB}=$ utility grate boiler, $\mathrm{CLC}=$ chemical looping combustion, $\mathrm{FBB}=$ fluidized bed boiler, TGA = thermogravimetric analysis, DTGA = differential thermogravimetric analysis, FBC = fluidized bed combustor, $\mathrm{CFD}=$ computational fluid dynamics); 6-System performance analysis (Ene. = energy, Ec. = economic, Env. = environmental, $\mathrm{Y}=$ analyzed, $\mathrm{N}=$ not analyzed); 7—Process modifications $\left(\mathrm{Y}=\right.$ performed, $\mathrm{Y}^{*}=$ performed only variations in biomass/waste share in feedstock, $\mathrm{N}=$ not performed); 8 -Operational issues ( $\mathrm{Y}=$ investigated, $\mathrm{N}=$ not investigated). Energy performance includes plant/equipment thermal efficiency and/or specific fuel consumption and its reduction. Economic performance includes NPV, TIC, breakeven point, and other suitable indicators. Environmental performance includes GHG emissions, other emissions, LCA and other related results. Process modifications include change in co-combustion ratio, particle size change, residence time change, oxycombustion, reburning, air excess and air staging. Operational issues include investigation/calculation of slagging and fouling tendencies, corrosion appearance, fuel burnout assessment, flame properties, ignition characteristics and ash/fly ash properties analyses.

\begin{tabular}{|c|c|c|c|c|c|c|c|c|c|c|c|}
\hline \multirow{2}{*}{ Ref. } & \multirow{2}{*}{1} & \multirow{2}{*}{2} & \multicolumn{2}{|c|}{3} & \multirow{2}{*}{4} & \multirow{2}{*}{5} & \multicolumn{3}{|c|}{6} & \multirow{2}{*}{7} & \multirow{2}{*}{8} \\
\hline & & & Fos. & $\mathbf{B}+\mathbf{W}$ & & & Ene. & Ec. & Env. & & \\
\hline [15] & $\mathrm{R}$ & 2001 & $M$ & $M$ & D, Ot. & FS & $Y$ & $Y$ & $\mathrm{Y}$ & $Y$ & $\mathrm{Y}$ \\
\hline [18] & $\mathrm{O}$ & 1996 & $S$ & S & $\mathrm{N}$ & FS (PPB) & $\mathrm{N}$ & $\mathrm{N}$ & $\mathrm{Y}$ & $\mathrm{Y}$ & Y \\
\hline [17] & $\mathrm{O}$ & 1998 & $\mathrm{M}$ & M & V & $\mathrm{M}$ & $\mathrm{Y}$ & $\mathrm{N}$ & $\mathrm{Y}$ & $\mathrm{Y}$ & $\mathrm{Y}$ \\
\hline [104] & $\mathrm{O}$ & 2000 & M & M & D, Ot. & $\mathrm{L}(\mathrm{F}+\mathrm{REB})$ & $\mathrm{N}$ & $\mathrm{N}$ & $\mathrm{Y}$ & $\mathrm{Y}$ & $\mathrm{N}$ \\
\hline [19] & $\mathrm{O}$ & 2000 & M & M & $\mathrm{D}$ & FS & $\mathrm{Y}$ & $\mathrm{N}$ & $\mathrm{Y}$ & $\mathrm{Y}$ & $\mathrm{Y}$ \\
\hline [105] & $\mathrm{O}$ & 2000 & M & $S$ & $\mathrm{~N}$ & FS (PPB) & $\mathrm{N}$ & $\mathrm{N}$ & Y & $\mathrm{Y}$ & $\mathrm{Y}$ \\
\hline [64] & $\mathrm{O}$ & 2000 & M & M & $\mathrm{N}$ & FS & $\mathrm{N}$ & $\mathrm{Y}$ & $\mathrm{Y}$ & $Y^{*}$ & $\mathrm{~N}$ \\
\hline [8] & $\mathrm{R}$ & 2001 & M & M & $\mathrm{V}$ & FS & $\mathrm{Y}$ & $\mathrm{N}$ & Y & $\mathrm{Y}$ & $\mathrm{Y}$ \\
\hline [65] & $\mathrm{O}$ & 2001 & $S$ & M & $\mathrm{N}$ & FS (PPB), C & $\mathrm{Y}$ & $\mathrm{N}$ & $\mathrm{Y}$ & $Y^{*}$ & $\mathrm{~N}$ \\
\hline [44] & $\mathrm{O}$ & 2002 & $S$ & M & $\mathrm{N}$ & FS (IB) & $\mathrm{N}$ & $\mathrm{N}$ & $\mathrm{Y}$ & $\mathrm{Y}$ & Y \\
\hline [82] & $\mathrm{O}$ & 2003 & $S$ & $S$ & $\mathrm{~N}$ & L (PFBA) & $\mathrm{N}$ & $\mathrm{N}$ & $\mathrm{Y}$ & $\mathrm{Y}$ & $\mathrm{Y}$ \\
\hline [106] & $\mathrm{O}$ & 2003 & $\mathrm{M}$ & $S$ & $\mathrm{~N}$ & $\mathrm{FS}(\mathrm{PCB})$ & $\mathrm{N}$ & $\mathrm{N}$ & $\mathrm{Y}$ & $\mathrm{Y}$ & $\mathrm{Y}$ \\
\hline [89] & $\mathrm{R}$ & 2003 & M & M & $\mathrm{N}$ & $\mathrm{M}$ & $\mathrm{N}$ & $\mathrm{N}$ & $\mathrm{Y}$ & $Y^{*}$ & $\mathrm{~N}$ \\
\hline [107] & $\mathrm{O}$ & 2005 & S & $S$ & $\mathrm{~N}$ & $\mathrm{P}, \mathrm{C}(\mathrm{PCB})$ & $\mathrm{N}$ & $\mathrm{N}$ & $\mathrm{Y}$ & $\mathrm{N}$ & $\mathrm{N}$ \\
\hline [37] & $\mathrm{O}$ & 2006 & S & M & $\mathrm{N}$ & $\mathrm{C}$ & Y & $\mathrm{N}$ & $\mathrm{Y}$ & $\mathrm{Y}$ & $\mathrm{Y}$ \\
\hline [108] & $\mathrm{O}$ & 2007 & $S$ & $S$ & $\mathrm{~N}$ & P, FS (CFBB) & $\mathrm{N}$ & $\mathrm{N}$ & $\mathrm{N}$ & $\mathrm{Y}$ & $\mathrm{N}$ \\
\hline [36] & $\mathrm{R}$ & 2007 & M & M & $\mathrm{D}$ & FS & $\mathrm{Y}$ & $\mathrm{Y}$ & $\mathrm{Y}$ & $\mathrm{Y}$ & $\mathrm{Y}$ \\
\hline [33] & $\mathrm{O}$ & 2007 & M & M & $\mathrm{N}$ & FS & $\mathrm{Y}$ & $\mathrm{N}$ & $\mathrm{Y}$ & $\mathrm{Y}$ & $\mathrm{N}$ \\
\hline [13] & $\mathrm{R}$ & 2008 & M & M & $\mathrm{N}$ & $\mathrm{FS}, \mathrm{C}$ & $\mathrm{Y}$ & $\mathrm{N}$ & $\mathrm{Y}$ & $\mathrm{Y}$ & Y \\
\hline [109] & $\mathrm{O}$ & 2008 & M & S & $\mathrm{N}$ & L (EFR) & $\mathrm{N}$ & $\mathrm{N}$ & $\mathrm{Y}$ & $\mathrm{Y}$ & Y \\
\hline [32] & $\mathrm{R}$ & 2010 & M & M & $\mathrm{N}$ & $\mathrm{M}-\mathrm{FS}$ & $\mathrm{N}$ & $\mathrm{N}$ & $\mathrm{Y}$ & $\mathrm{Y}$ & $\mathrm{Y}$ \\
\hline [110] & $\mathrm{O}$ & 2011 & $S$ & M & $\mathrm{N}$ & $\mathrm{L}, \mathrm{C}(\mathrm{FBB}+\mathrm{PY}+\mathrm{REB})$ & $\mathrm{N}$ & $\mathrm{N}$ & Y & $\mathrm{Y}$ & $\mathrm{N}$ \\
\hline [111] & $\mathrm{O}$ & 2011 & M & $S$ & $\mathrm{~N}$ & $\mathrm{~L}(\mathrm{~F}+\mathrm{REB})$ & $\mathrm{N}$ & $\mathrm{N}$ & $\mathrm{Y}$ & $\mathrm{Y}$ & $\mathrm{Y}$ \\
\hline [51] & $\mathrm{R}$ & 2012 & M & M & $\mathrm{N}$ & $\mathrm{M}-\mathrm{FS}$ & $\mathrm{N}$ & $\mathrm{N}$ & $\mathrm{Y}$ & $\mathrm{N}$ & $\mathrm{Y}$ \\
\hline [112] & $\mathrm{O}$ & 2012 & $S$ & M & G & $\mathrm{C}(\mathrm{BG}+\mathrm{REB})$ & $\mathrm{N}$ & $\mathrm{N}$ & $\mathrm{Y}$ & $\mathrm{Y}$ & $\mathrm{N}$ \\
\hline [113] & $\mathrm{O}$ & 2012 & $S$ & $S$ & $\mathrm{~N}$ & $\mathrm{~L}(\mathrm{BF})$ & $\mathrm{N}$ & $\mathrm{N}$ & $\mathrm{Y}$ & $Y^{*}$ & $\mathrm{~N}$ \\
\hline [114] & $\mathrm{O}$ & 2012 & M & M & $\mathrm{N}$ & $\mathrm{FS}, \mathrm{C}$ & $\mathrm{Y}$ & $\mathrm{N}$ & $\mathrm{Y}$ & $\mathrm{Y}$ & $\mathrm{Y}$ \\
\hline [115] & $\mathrm{O}$ & 2012 & M & $\mathrm{M}$ & $\mathrm{N}$ & $\mathrm{P}, \mathrm{C}$ & $\mathrm{Y}$ & $\mathrm{N}$ & $\mathrm{Y}$ & $\mathrm{Y}$ & $\mathrm{N}$ \\
\hline [116] & $\mathrm{O}$ & 2012 & M & $S$ & $\mathrm{~N}$ & L (EFR) & $\mathrm{N}$ & $\mathrm{N}$ & $\mathrm{Y}$ & $\mathrm{Y}$ & $\mathrm{Y}$ \\
\hline [117] & $\mathrm{O}$ & 2013 & M & $S$ & $\mathrm{~N}$ & L (TGA, DTGA) & $\mathrm{N}$ & $\mathrm{N}$ & $\mathrm{N}$ & $\mathrm{Y}$ & $\mathrm{N}$ \\
\hline [118] & $\mathrm{O}$ & 2013 & M & M & Ot. & L (TGA, DTGA) & $\mathrm{N}$ & $\mathrm{N}$ & $\mathrm{N}$ & $\mathrm{Y}$ & $\mathrm{N}$ \\
\hline [68] & $\mathrm{O}$ & 2013 & $S$ & $S$ & $\mathrm{~N}$ & FS, C & $\mathrm{Y}$ & $\mathrm{Y}$ & $\mathrm{Y}$ & $Y^{*}$ & $\mathrm{~N}$ \\
\hline [119] & $\mathrm{O}$ & 2013 & $S$ & S & G & $\mathrm{P}(\mathrm{BG}+\mathrm{REB})$ & $\mathrm{Y}$ & $\mathrm{N}$ & $\mathrm{Y}$ & $\mathrm{Y}$ & $\mathrm{N}$ \\
\hline [57] & $\mathrm{O}$ & 2013 & $S$ & $S$ & $\mathrm{~N}$ & FS, C & $\mathrm{Y}$ & $\mathrm{N}$ & $\mathrm{N}$ & $\mathrm{Y}$ & $\mathrm{Y}$ \\
\hline [120] & $\mathrm{O}$ & 2013 & M & M & $\mathrm{D}$ & $\mathrm{L}, \mathrm{FS}$ (PPB) & $\mathrm{N}$ & $\mathrm{N}$ & $\mathrm{Y}$ & $\mathrm{Y}$ & $\mathrm{Y}$ \\
\hline
\end{tabular}


Table 5. Cont.

\begin{tabular}{|c|c|c|c|c|c|c|c|c|c|c|c|}
\hline \multirow{2}{*}{ Ref. } & \multirow{2}{*}{1} & \multirow{2}{*}{2} & \multicolumn{2}{|c|}{3} & \multirow{2}{*}{4} & \multirow{2}{*}{5} & \multicolumn{3}{|c|}{6} & \multirow{2}{*}{7} & \multirow{2}{*}{8} \\
\hline & & & Fos. & $\mathbf{B}+\mathbf{W}$ & & & Ene. & Ec. & Env. & & \\
\hline [31] & $\mathrm{R}$ & 2014 & $\mathrm{M}$ & $\mathrm{M}$ & $\mathrm{T}, \mathrm{G}, \mathrm{Ot}$ & $\mathrm{M}-\mathrm{FS}(\mathrm{BG}+\mathrm{REB})$ & $\mathrm{N}$ & $\mathrm{N}$ & $Y$ & $\mathrm{Y}$ & $\mathrm{Y}$ \\
\hline [121] & $\mathrm{O}$ & 2014 & $S$ & M & $\mathrm{N}$ & $\mathrm{L}(\mathrm{DTF})$ & $\mathrm{N}$ & $\mathrm{N}$ & $\mathrm{Y}$ & $\mathrm{Y}$ & $\mathrm{Y}$ \\
\hline [11] & $\mathrm{R}$ & 2014 & M & M & $\mathrm{T}, \mathrm{G}, \mathrm{Ot}$ & $\mathrm{M}-\mathrm{L}$ & $\mathrm{N}$ & $\mathrm{N}$ & $\mathrm{Y}$ & $\mathrm{N}$ & $\mathrm{Y}$ \\
\hline [10] & $\mathrm{R}$ & 2014 & M & M & $\mathrm{V}$ & $\mathrm{FS}(\mathrm{BG}+\mathrm{PY})$ & $\mathrm{N}$ & $\mathrm{N}$ & Y & $\mathrm{Y}$ & $\mathrm{Y}$ \\
\hline [86] & $\mathrm{O}$ & 2015 & $S$ & M & $\mathrm{N}$ & $\mathrm{L}(\mathrm{IFR})$ & $\mathrm{N}$ & $\mathrm{N}$ & $\mathrm{Y}$ & $\mathrm{Y}$ & $\mathrm{Y}$ \\
\hline [122] & $\mathrm{O}$ & 2015 & M & $S$ & $\mathrm{~N}$ & $\mathrm{~L}(\mathrm{CFBC})$ & $\mathrm{N}$ & $\mathrm{N}$ & $\mathrm{N}$ & $\mathrm{Y}$ & $\mathrm{Y}$ \\
\hline [123] & $\mathrm{O}$ & 2015 & $\mathrm{~S}$ & S & $\mathrm{N}$ & $\mathrm{FS}, \mathrm{C}(\mathrm{PPB}+\mathrm{CFD})$ & $\mathrm{N}$ & $\mathrm{N}$ & $\mathrm{N}$ & $\mathrm{Y}$ & $\mathrm{Y}$ \\
\hline [124] & $\mathrm{O}$ & 2015 & $S$ & $S$ & $\mathrm{~N}$ & $\mathrm{~L}, \mathrm{C}(\mathrm{F}+\mathrm{CFD})$ & $\mathrm{N}$ & $\mathrm{N}$ & $\mathrm{N}$ & $\mathrm{Y}$ & $\mathrm{Y}$ \\
\hline [41] & $\mathrm{O}$ & 2015 & S & M & $\mathrm{N}$ & $\mathrm{L}(\mathrm{CFBC})$ & $\mathrm{N}$ & $\mathrm{N}$ & $\mathrm{Y}$ & $\mathrm{Y}$ & $\mathrm{N}$ \\
\hline [125] & $\mathrm{O}$ & 2016 & S & $S$ & $\mathrm{~N}$ & $\mathrm{~L}(\mathrm{~F}+\mathrm{REB})$ & $\mathrm{N}$ & $\mathrm{N}$ & $\mathrm{Y}$ & $\mathrm{Y}$ & $\mathrm{Y}$ \\
\hline [25] & $\mathrm{O}$ & 2016 & $S$ & M & $\mathrm{N}$ & L (TGA, DTGA) & $\mathrm{N}$ & $\mathrm{N}$ & $\mathrm{N}$ & $\mathrm{Y}$ & $\mathrm{Y}$ \\
\hline [38] & $\mathrm{O}$ & 2016 & $S$ & M & $\mathrm{D}$ & L (DTF) & $\mathrm{N}$ & $\mathrm{N}$ & $\mathrm{N}$ & $\mathrm{Y}$ & $\mathrm{Y}$ \\
\hline [49] & $\mathrm{O}$ & 2016 & M & $S$ & $\mathrm{~N}$ & $\mathrm{~L}(\mathrm{PFF})$ & $\mathrm{N}$ & $\mathrm{N}$ & $\mathrm{Y}$ & $\mathrm{Y}$ & $\mathrm{Y}$ \\
\hline [69] & $\mathrm{O}$ & 2016 & M & M & $\mathrm{N}$ & FS & $\mathrm{Y}$ & $\mathrm{Y}$ & $\mathrm{Y}$ & $Y^{*}$ & $\mathrm{~N}$ \\
\hline [55] & $\mathrm{O}$ & 2016 & M & M & $\mathrm{N}$ & FS, C & $\mathrm{Y}$ & $\mathrm{Y}$ & $\mathrm{Y}$ & $Y^{*}$ & $\mathrm{~N}$ \\
\hline [126] & $\mathrm{O}$ & 2016 & M & $S$ & $\mathrm{~N}$ & L (EFR) & $\mathrm{N}$ & $\mathrm{N}$ & $\mathrm{Y}$ & $\mathrm{Y}$ & $\mathrm{Y}$ \\
\hline [58] & $\mathrm{O}$ & 2017 & M & $S$ & $\mathrm{~N}$ & $\mathrm{FS}, \mathrm{C}(\mathrm{CB}+\mathrm{CFD})$ & $\mathrm{N}$ & $\mathrm{N}$ & $\mathrm{Y}$ & $\mathrm{N}$ & $\mathrm{N}$ \\
\hline [127] & $\mathrm{O}$ & 2017 & $S$ & M & $\mathrm{T}$ & $\mathrm{L}(\mathrm{FBC})$ & $\mathrm{N}$ & $\mathrm{N}$ & $\mathrm{Y}$ & $\mathrm{Y}$ & $\mathrm{Y}$ \\
\hline [103] & $\mathrm{R}$ & 2017 & M & M & $\mathrm{V}$ & $\mathrm{M}-\mathrm{FS}, \mathrm{L}$ & $\mathrm{N}$ & $\mathrm{N}$ & $\mathrm{Y}$ & $\mathrm{Y}$ & $\mathrm{Y}$ \\
\hline [78] & $\mathrm{O}$ & 2017 & $S$ & $S$ & $\mathrm{D}$ & L (TGA, DTGA) & $\mathrm{N}$ & $\mathrm{N}$ & $\mathrm{N}$ & $\mathrm{Y}$ & $\mathrm{N}$ \\
\hline [85] & $\mathrm{O}$ & 2017 & $M$ & S & $\mathrm{N}$ & $\mathrm{P}$ & $\mathrm{Y}$ & $\mathrm{N}$ & $\mathrm{Y}$ & $\mathrm{Y}$ & $\mathrm{Y}$ \\
\hline$[84]$ & $\mathrm{O}$ & 2017 & $S$ & $S$ & $\mathrm{~T}$ & $\mathrm{P}$ & $\mathrm{Y}$ & $\mathrm{N}$ & $\mathrm{Y}$ & $\mathrm{Y}$ & $\mathrm{N}$ \\
\hline [128] & $\mathrm{O}$ & 2017 & M & $S$ & $\mathrm{~N}$ & $\mathrm{~L}, \mathrm{P}(\mathrm{PFC})$ & $\mathrm{N}$ & $\mathrm{N}$ & $\mathrm{N}$ & $\mathrm{Y}$ & $\mathrm{Y}$ \\
\hline [129] & $\mathrm{O}$ & 2017 & $S$ & S & $\mathrm{D}$ & FS, C & Y & $\mathrm{N}$ & $\mathrm{Y}$ & $\mathrm{Y}$ & $\mathrm{Y}$ \\
\hline [56] & $\mathrm{O}$ & 2017 & $S$ & $S$ & $\mathrm{~V}$ & FS & Y & $\mathrm{N}$ & $\mathrm{Y}$ & $Y^{*}$ & $\mathrm{~N}$ \\
\hline [52] & $\mathrm{R}$ & 2017 & M & $\mathrm{M}$ & $\mathrm{D}$ & $\mathrm{M}-\mathrm{FS}$ & $\mathrm{N}$ & $\mathrm{N}$ & $\mathrm{Y}$ & $\mathrm{Y}^{*}$ & $\mathrm{~N}$ \\
\hline [42] & $\mathrm{O}$ & 2018 & $S$ & $S$ & $\mathrm{~N}$ & $\mathrm{~L}(\mathrm{CFBC})$ & $\mathrm{N}$ & $\mathrm{N}$ & $\mathrm{Y}$ & $\mathrm{Y}$ & $\mathrm{Y}$ \\
\hline [20] & $\mathrm{O}$ & 2018 & S & $S$ & $\mathrm{D}$ & FS, C & $\mathrm{Y}$ & $\mathrm{N}$ & $\mathrm{Y}$ & $\mathrm{N}$ & $\mathrm{Y}$ \\
\hline [26] & $\mathrm{O}$ & 2018 & $S$ & $S$ & $\mathrm{D}, \mathrm{T}$ & L (TGA, DTGA) & $\mathrm{N}$ & $\mathrm{N}$ & $\mathrm{N}$ & $\mathrm{Y}$ & $\mathrm{Y}$ \\
\hline [130] & $\mathrm{O}$ & 2018 & $S$ & S & $\mathrm{N}$ & C & $\mathrm{Y}$ & $\mathrm{N}$ & $\mathrm{Y}$ & $\mathrm{N}$ & $\mathrm{N}$ \\
\hline [131] & $\mathrm{O}$ & 2018 & $S$ & M & $\mathrm{T}$ & $\mathrm{L}(\mathrm{DTF})$ & $\mathrm{N}$ & $\mathrm{N}$ & $\mathrm{N}$ & $\mathrm{N}$ & $\mathrm{Y}$ \\
\hline [132] & $\mathrm{O}$ & 2018 & M & M & $\mathrm{T}$ & $\mathrm{L}$ & Y & $\mathrm{N}$ & $\mathrm{Y}$ & $\mathrm{Y}$ & $\mathrm{Y}$ \\
\hline [91] & $\mathrm{O}$ & 2018 & $S$ & M & $\mathrm{T}$ & L (SPR) & $\mathrm{N}$ & $\mathrm{N}$ & $\mathrm{Y}$ & $\mathrm{Y}$ & $\mathrm{Y}$ \\
\hline [22] & $\mathrm{O}$ & 2018 & $S$ & M & $\mathrm{D}$ & $\mathrm{L}(\mathrm{DTF}+\mathrm{TGA})$ & $\mathrm{N}$ & $\mathrm{N}$ & $\mathrm{Y}$ & $\mathrm{Y}$ & $\mathrm{Y}$ \\
\hline [133] & $\mathrm{O}$ & 2018 & $S$ & M & $\mathrm{N}$ & $\mathrm{P}(\mathrm{F})$ & $\mathrm{N}$ & $\mathrm{N}$ & $\mathrm{Y}$ & $\mathrm{Y}$ & $\mathrm{Y}$ \\
\hline [134] & $\mathrm{O}$ & 2018 & $S$ & $S$ & $\mathrm{~N}$ & $\mathrm{~L}(\mathrm{DTF}+\mathrm{TGA})$ & $\mathrm{N}$ & $\mathrm{N}$ & $\mathrm{Y}$ & $\mathrm{N}$ & $\mathrm{Y}$ \\
\hline [71] & $\mathrm{O}$ & 2018 & M & M & $\mathrm{D}$ & $\mathrm{L}$ & $\mathrm{Y}$ & $\mathrm{Y}$ & $\mathrm{Y}$ & $\mathrm{Y}$ & $\mathrm{N}$ \\
\hline [45] & $\mathrm{O}$ & 2018 & $\mathrm{~S}$ & S & $\mathrm{N}$ & $\mathrm{P}(\mathrm{PFC})$ & $\mathrm{N}$ & $\mathrm{N}$ & $\mathrm{Y}$ & $\mathrm{N}$ & $\mathrm{Y}$ \\
\hline$[50]$ & $\mathrm{O}$ & 2018 & $S$ & M & $\mathrm{D}$ & L (TGA, DTGA) & $\mathrm{N}$ & $\mathrm{N}$ & $\mathrm{N}$ & $\mathrm{Y}$ & $\mathrm{Y}$ \\
\hline [135] & $\mathrm{O}$ & 2018 & $S$ & $S$ & $\mathrm{~N}$ & FS (IB-MG) & $\mathrm{N}$ & $\mathrm{N}$ & $\mathrm{Y}$ & $Y^{*}$ & $\mathrm{~N}$ \\
\hline [63] & $\mathrm{O}$ & 2018 & S & $S$ & $\mathrm{D}$ & FS, C & $\mathrm{Y}$ & $\mathrm{Y}$ & $\mathrm{N}$ & $\mathrm{Y}$ & $\mathrm{N}$ \\
\hline [136] & $\mathrm{O}$ & 2018 & M & $S$ & $\mathrm{~N}$ & $\mathrm{~L}(\mathrm{PFF})$ & $\mathrm{N}$ & $\mathrm{N}$ & $\mathrm{Y}$ & $\mathrm{Y}$ & $\mathrm{Y}$ \\
\hline [23] & $\mathrm{O}$ & 2018 & M & M & $\mathrm{N}$ & $\mathrm{L}$ (DFT) & $\mathrm{N}$ & $\mathrm{N}$ & $\mathrm{Y}$ & $\mathrm{Y}$ & $\mathrm{Y}$ \\
\hline [28] & $\mathrm{O}$ & 2018 & M & S & $\mathrm{N}$ & $\mathrm{P}(\mathrm{PFC})$ & $\mathrm{N}$ & $\mathrm{N}$ & $\mathrm{N}$ & $\mathrm{Y}$ & $\mathrm{Y}$ \\
\hline [137] & $\mathrm{O}$ & 2018 & - & M & $\mathrm{N}$ & $\mathrm{M}, \mathrm{C}$ & $\mathrm{N}$ & $\mathrm{N}$ & $\mathrm{N}$ & $\mathrm{Y}$ & $\mathrm{Y}$ \\
\hline [138] & $\mathrm{O}$ & 2018 & $S$ & $S$ & $\mathrm{~N}$ & $\mathrm{~L}(\mathrm{FBC}+\mathrm{TGA}, \mathrm{DTGA})$ & $\mathrm{N}$ & $\mathrm{N}$ & $\mathrm{Y}$ & $\mathrm{Y}$ & $\mathrm{Y}$ \\
\hline [70] & $\mathrm{O}$ & 2018 & $S$ & $\mathrm{M}$ & $\mathrm{T}$ & FS, C & Y & $\mathrm{Y}$ & $\mathrm{Y}$ & $Y^{*}$ & $\mathrm{~N}$ \\
\hline [139] & $\mathrm{O}$ & 2019 & $\mathrm{~S}$ & $\mathrm{~S}$ & $\mathrm{D}$ & L (DTF) & $\mathrm{N}$ & $\mathrm{N}$ & $\mathrm{N}$ & $\mathrm{Y}$ & $\mathrm{Y}$ \\
\hline [140] & $\mathrm{R}$ & 2019 & M & M & $\mathrm{N}$ & $\mathrm{M}, \mathrm{C}(\mathrm{CLC})$ & $\mathrm{N}$ & $\mathrm{N}$ & $\mathrm{Y}$ & $\mathrm{Y}$ & $\mathrm{Y}$ \\
\hline [47] & $\mathrm{O}$ & 2019 & M & M & $\mathrm{D}$ & L (TGA, DTGA) & $\mathrm{N}$ & $\mathrm{N}$ & $\mathrm{N}$ & $\mathrm{Y}$ & $\mathrm{Y}$ \\
\hline [141] & $\mathrm{O}$ & 2019 & $S$ & $S$ & $\mathrm{D}$ & $\mathrm{L}, \mathrm{C}(\mathrm{TGA})$ & $\mathrm{N}$ & $\mathrm{N}$ & $\mathrm{N}$ & $\mathrm{Y}$ & $\mathrm{Y}$ \\
\hline [39] & $\mathrm{O}$ & 2019 & M & M & $\mathrm{N}$ & FS (CFBB) & $\mathrm{N}$ & $\mathrm{N}$ & $\mathrm{N}$ & $\mathrm{Y}$ & $\mathrm{Y}$ \\
\hline [142] & $\mathrm{R}$ & 2019 & M & M & $\mathrm{N}$ & $\mathrm{M}, \mathrm{C}$ & $\mathrm{Y}$ & $\mathrm{Y}$ & Y & $\mathrm{Y}$ & $\mathrm{Y}$ \\
\hline [143] & $\mathrm{O}$ & 2019 & $S$ & M & Ot. & L (TGA, DTGA) & $\mathrm{N}$ & $\mathrm{N}$ & $\mathrm{N}$ & $\mathrm{Y}$ & $\mathrm{Y}$ \\
\hline [43] & $\mathrm{O}$ & 2019 & $S$ & $S$ & $\mathrm{D}$ & $\mathrm{L}, \mathrm{P}, \mathrm{C}(\mathrm{DTF}+\mathrm{CFBB})$ & $\mathrm{N}$ & $\mathrm{N}$ & $\mathrm{Y}$ & $\mathrm{Y}$ & $\mathrm{Y}$ \\
\hline [83] & $\mathrm{O}$ & 2019 & $S$ & S & $\mathrm{N}$ & L (TGA, DTGA) & $\mathrm{N}$ & $\mathrm{N}$ & $\mathrm{N}$ & $\mathrm{Y}$ & $\mathrm{Y}$ \\
\hline [144] & $\mathrm{O}$ & 2019 & $\mathrm{~S}$ & S & $\mathrm{N}$ & $\mathrm{P}(\mathrm{F})$ & $\mathrm{N}$ & $\mathrm{N}$ & $\mathrm{N}$ & $\mathrm{N}$ & $\mathrm{Y}$ \\
\hline
\end{tabular}


Table 5. Cont

\begin{tabular}{|c|c|c|c|c|c|c|c|c|c|c|c|}
\hline \multirow{2}{*}{ Ref. } & \multirow{2}{*}{1} & \multirow{2}{*}{2} & \multicolumn{2}{|c|}{3} & \multirow{2}{*}{4} & \multirow{2}{*}{5} & \multicolumn{3}{|c|}{6} & \multirow{2}{*}{7} & \multirow{2}{*}{8} \\
\hline & & & Fos. & $\mathbf{B}+\mathbf{W}$ & & & Ene. & Ec. & Env. & & \\
\hline [145] & $\mathrm{O}$ & 2019 & $S$ & M & $\mathrm{N}$ & FS, C (ash samples) & $\mathrm{N}$ & $\mathrm{N}$ & $\mathrm{N}$ & $\mathrm{Y}$ & $\mathrm{Y}$ \\
\hline [90] & $\mathrm{O}$ & 2019 & S & $S$ & $\mathrm{~N}$ & L (MIA-ash samples) & $\mathrm{N}$ & $\mathrm{N}$ & $\mathrm{N}$ & $\mathrm{Y}$ & Y \\
\hline [46] & $\mathrm{O}$ & 2019 & S & S & $\mathrm{D}$ & L (TGA, DTGA) & $\mathrm{N}$ & $\mathrm{N}$ & $\mathrm{Y}$ & $\mathrm{Y}$ & $\mathrm{Y}$ \\
\hline [146] & $\mathrm{O}$ & 2019 & $S$ & $S$ & $\mathrm{~N}$ & $\mathrm{M}, \mathrm{C}(\mathrm{CFD})$ & $\mathrm{N}$ & $\mathrm{N}$ & $\mathrm{Y}$ & $\mathrm{N}$ & $\mathrm{Y}$ \\
\hline [147] & $\mathrm{O}$ & 2019 & $S$ & M & $\mathrm{D}$ & L (DTF) & $\mathrm{N}$ & $\mathrm{N}$ & $\mathrm{Y}$ & $\mathrm{Y}$ & $\mathrm{Y}$ \\
\hline [148] & $\mathrm{O}$ & 2019 & $S$ & $S$ & $\mathrm{D}$ & L (TGA, DTGA) & $\mathrm{N}$ & $\mathrm{N}$ & $\mathrm{N}$ & $\mathrm{N}$ & $\mathrm{Y}$ \\
\hline$[88]$ & $\mathrm{O}$ & 2019 & $\mathrm{M}$ & M & $\mathrm{N}$ & L (MIA) & $\mathrm{N}$ & $\mathrm{N}$ & $\mathrm{N}$ & $\mathrm{N}$ & $\mathrm{Y}$ \\
\hline$[24]$ & $\mathrm{O}$ & 2019 & $\mathrm{M}$ & $S$ & $\mathrm{~N}$ & $\mathrm{~L}(\mathrm{DTF}+\mathrm{TGA})$ & $\mathrm{N}$ & $\mathrm{N}$ & $\mathrm{N}$ & $\mathrm{Y}$ & $\mathrm{Y}$ \\
\hline [149] & $\mathrm{O}$ & 2020 & S & S & $\mathrm{T}$ & $\mathrm{P}(\mathrm{PFF})$ & $\mathrm{N}$ & $\mathrm{N}$ & $\mathrm{Y}$ & $\mathrm{Y}$ & $\mathrm{Y}$ \\
\hline [150] & $\mathrm{O}$ & 2020 & S & M & $\mathrm{D}$ & L (MIA) & $\mathrm{N}$ & $\mathrm{N}$ & $\mathrm{N}$ & $\mathrm{Y}$ & $\mathrm{Y}$ \\
\hline [29] & $\mathrm{O}$ & 2020 & S & $S$ & $\mathrm{~N}$ & $\mathrm{~L}(\mathrm{FBC})$ & $\mathrm{N}$ & $\mathrm{N}$ & $\mathrm{Y}$ & $\mathrm{Y}$ & $\mathrm{Y}$ \\
\hline [59] & $\mathrm{O}$ & 2020 & $S$ & S & $\mathrm{N}$ & $\mathrm{FS}, \mathrm{C}(\mathrm{FBC}+\mathrm{CFD})$ & $\mathrm{N}$ & $\mathrm{N}$ & $\mathrm{Y}$ & $\mathrm{Y}$ & $\mathrm{Y}$ \\
\hline [151] & $\mathrm{O}$ & 2020 & $S$ & $S$ & $\mathrm{~N}$ & FS, C (UGB + REB) & $\mathrm{N}$ & $\mathrm{N}$ & $\mathrm{Y}$ & $\mathrm{Y}$ & $\mathrm{Y}$ \\
\hline [152] & $\mathrm{O}$ & 2020 & $S$ & M & $\mathrm{N}$ & $\mathrm{L}, \mathrm{C}(\mathrm{CLC}+\mathrm{CFD})$ & $\mathrm{N}$ & $\mathrm{N}$ & $\mathrm{Y}$ & $\mathrm{Y}$ & $\mathrm{Y}$ \\
\hline$[21]$ & $\mathrm{O}$ & 2020 & $S$ & M & $\mathrm{N}$ & (pelletizing grate kiln) $\mathrm{P}, \mathrm{C}$ & $\mathrm{N}$ & $\mathrm{N}$ & $\mathrm{N}$ & $\mathrm{Y}$ & $\mathrm{Y}$ \\
\hline [153] & $\mathrm{O}$ & 2020 & $S$ & $S$ & $\mathrm{~N}$ & $\mathrm{~L}, \mathrm{C}(\mathrm{F}+\mathrm{CFD})$ & $\mathrm{N}$ & $\mathrm{N}$ & $\mathrm{Y}$ & $\mathrm{Y}$ & $\mathrm{Y}$ \\
\hline$[40]$ & $\mathrm{O}$ & 2020 & $S$ & $S$ & $\mathrm{D}$ & L (MIA—ash samples) & $\mathrm{N}$ & $\mathrm{N}$ & $\mathrm{N}$ & $\mathrm{Y}$ & $\mathrm{Y}$ \\
\hline [154] & $\mathrm{O}$ & 2020 & $S$ & S & $\mathrm{T}$ & L (TGA, DTGA) & $\mathrm{N}$ & $\mathrm{N}$ & $\mathrm{N}$ & $\mathrm{N}$ & $\mathrm{Y}$ \\
\hline$[27]$ & $\mathrm{O}$ & 2020 & M & M & $\mathrm{N}$ & L (TGA, DTGA) & $\mathrm{N}$ & $\mathrm{N}$ & $\mathrm{N}$ & $\mathrm{Y}$ & $\mathrm{Y}$ \\
\hline [155] & $\mathrm{O}$ & 2020 & $S$ & M & $\mathrm{T}$ & $\mathrm{P}(\mathrm{PFC})$ & $\mathrm{N}$ & $\mathrm{N}$ & $\mathrm{Y}$ & $Y^{*}$ & $\mathrm{~N}$ \\
\hline [48] & $\mathrm{O}$ & 2020 & S & M & $\mathrm{N}$ & $\mathrm{L}(\mathrm{TF})$ & $\mathrm{N}$ & $\mathrm{N}$ & $\mathrm{Y}$ & $\mathrm{Y}$ & $\mathrm{Y}$ \\
\hline [156] & $\mathrm{O}$ & 2020 & $S$ & M & $\mathrm{N}$ & L (TGA, DTGA) & $\mathrm{N}$ & $\mathrm{N}$ & $\mathrm{Y}$ & $Y^{*}$ & $\mathrm{Y}$ \\
\hline [157] & $\mathrm{O}$ & 2020 & M & M & $\mathrm{N}$ & L (TGA, DTGA, TF) & $\mathrm{N}$ & $\mathrm{N}$ & $\mathrm{Y}$ & $Y^{*}$ & $\mathrm{Y}$ \\
\hline [158] & $\mathrm{O}$ & 2020 & $S$ & $S$ & $\mathrm{~N}$ & L (MIA) & $\mathrm{N}$ & $\mathrm{N}$ & $\mathrm{N}$ & $Y^{*}$ & $\mathrm{Y}$ \\
\hline
\end{tabular}

Table 6. Summary of relevant papers providing information about biomass, waste, and fossil fuels preparation and their properties. Legend: 1 -Feed preparation $(\mathrm{M}-$ milling, $\mathrm{P}$-pelletizing, $\mathrm{S}$ - sieving, $\mathrm{Sh}=$ shredding, $\mathrm{N}-\mathrm{none}, \mathrm{V}-$ various), 2-Proximate analysis, 3-Elemental analysis, 4-LHV $/ \mathrm{HHV}$ estimation $(\mathrm{Y}=$ provided, $\mathrm{N}=$ not provided $), \mathrm{RDF}=$ refuse-derived fuel, NG = natural gas. $\mathrm{LHV}=$ lower heating value, $\mathrm{HHV}=$ higher heating value.

\begin{tabular}{|c|c|c|c|c|c|c|c|c|c|c|}
\hline \multirow{2}{*}{ Ref. } & \multicolumn{4}{|c|}{ Fossil Fuel } & \multicolumn{6}{|c|}{ Biomass/Waste } \\
\hline & Description & 1 & 2 & 3 & 4 & Description & 1 & 2 & 3 & 4 \\
\hline [18] & High sulfur bituminous coal & $\mathrm{M}$ & $\mathrm{Y}$ & $\mathrm{Y}$ & $\mathrm{Y}$ & Wheat straw & Sh, $\mathrm{P}$ & $\mathrm{Y}$ & $\mathrm{Y}$ & $\bar{Y}$ \\
\hline [104] & Coal, natural gas & M & $\mathrm{Y}$ & $\mathrm{Y}$ & $\mathrm{Y}$ & Hard- and softwood & Sh, S & Y & $\mathrm{Y}$ & $\mathrm{Y}$ \\
\hline [19] & Coal, oil & $\mathrm{V}$ & $\mathrm{Y}$ & $\mathrm{Y}$ & $\mathrm{Y}$ & Various biomass types + sources & $\mathrm{V}$ & $\mathrm{Y}$ & $\mathrm{Y}$ & $\mathrm{Y}$ \\
\hline [105] & Two types of coal & M & $\mathrm{Y}$ & $\mathrm{Y}$ & $\mathrm{Y}$ & Surplus straw from farming & Sh, M & $\mathrm{Y}$ & $\mathrm{Y}$ & $\mathrm{Y}$ \\
\hline [8] & Coals & $\mathrm{V}$ & $\mathrm{Y}$ & $\mathrm{Y}$ & $\mathrm{Y}$ & Multiple biomass and waste types & V & $\mathrm{Y}$ & $\mathrm{Y}$ & $\mathrm{Y}$ \\
\hline [65] & Coal Illinois No. 6 & $\mathrm{~N}$ & $\mathrm{~N}$ & $\mathrm{~N}$ & $\mathrm{~N}$ & Multiple biomass types & $\mathrm{N}$ & $\mathrm{Y}$ & $\mathrm{Y}$ & $\mathrm{Y}$ \\
\hline$[44]$ & Greek lignite & $\mathrm{N}$ & $\mathrm{Y}$ & $\mathrm{Y}$ & $\mathrm{Y}$ & Multiple biomass and waste types & $\mathrm{N}$ & $\mathrm{Y}$ & $\mathrm{Y}$ & $\mathrm{Y}$ \\
\hline [82] & Czech Sokolov coal & $S$ & $\mathrm{Y}$ & $\mathrm{Y}$ & $\mathrm{Y}$ & Wood chips & Sh, $S$ & $\mathrm{Y}$ & $\mathrm{Y}$ & $\mathrm{Y}$ \\
\hline [106] & Polish and Russian coal & M & $\mathrm{Y}$ & $\mathrm{Y}$ & Y & Pine sawdust & $\mathrm{N}$ & Y & $\mathrm{Y}$ & Y \\
\hline [89] & Multiple coal types & $\mathrm{N}$ & $\mathrm{Y}$ & $\mathrm{Y}$ & $\mathrm{Y}$ & Multiple biomass types & $\mathrm{N}$ & $\mathrm{Y}$ & $\mathrm{Y}$ & $\mathrm{Y}$ \\
\hline [107] & Bituminous UK coal & M & $\mathrm{Y}$ & $\mathrm{Y}$ & $\mathrm{N}$ & Pinewood pellets & $M, S$ & Y & $\mathrm{Y}$ & $\mathrm{N}$ \\
\hline [37] & Upper Silesian coal & $\mathrm{N}$ & Y & $\mathrm{N}$ & $\mathrm{Y}$ & Wood, straw, sewage sludge & $\mathrm{N}$ & $\mathrm{Y}$ & $\mathrm{N}$ & $\mathrm{Y}$ \\
\hline [108] & Coal & $\mathrm{N}$ & $\mathrm{Y}$ & $\mathrm{Y}$ & $\mathrm{N}$ & Wood chips & $\mathrm{N}$ & $\mathrm{Y}$ & $\mathrm{Y}$ & $\mathrm{N}$ \\
\hline$[36]$ & Multiple coal types & $\mathrm{N}$ & $\mathrm{Y}$ & $\mathrm{Y}$ & $\mathrm{Y}$ & Multiple biomass and waste types & $\mathrm{N}$ & $\mathrm{Y}$ & $\mathrm{Y}$ & $\mathrm{Y}$ \\
\hline [33] & Lignite and bituminous coal & M & $\mathrm{Y}$ & $\mathrm{Y}$ & $\mathrm{N}$ & Multiple biomass types & M & $\mathrm{Y}$ & $\mathrm{Y}$ & $\mathrm{N}$ \\
\hline [13] & Multiple coal types & M & $\mathrm{Y}$ & $\mathrm{Y}$ & $\mathrm{Y}$ & Multiple biomass and waste types & M & Y & $\mathrm{Y}$ & $\mathrm{Y}$ \\
\hline$[109]$ & Four coal types & M, S & $\mathrm{Y}$ & $\mathrm{Y}$ & $\mathrm{N}$ & Eucalyptus biomass & M, S & $\mathrm{Y}$ & $\mathrm{Y}$ & $\mathrm{N}$ \\
\hline [111] & NG, coal & $\mathrm{N}$ & $\mathrm{Y}$ & $\mathrm{Y}$ & $\mathrm{Y}$ & Pine sawdust & $\mathrm{N}$ & $\mathrm{Y}$ & $\mathrm{Y}$ & $\mathrm{Y}$ \\
\hline$[112]$ & Hard coal & $\mathrm{N}$ & Y & $\mathrm{Y}$ & $\mathrm{N}$ & Sewage sludge, two types & $\mathrm{N}$ & $\mathrm{Y}$ & $\mathrm{Y}$ & $\mathrm{N}$ \\
\hline [113] & Coal Pittsburgh No. 8 & M, S & $\mathrm{Y}$ & $\mathrm{Y}$ & Y & Sugarcane bagasse & M, S & $\mathrm{Y}$ & $\mathrm{Y}$ & $\mathrm{Y}$ \\
\hline$[114]$ & Two coal types & $\mathrm{N}$ & $\mathrm{Y}$ & $\mathrm{Y}$ & $\mathrm{Y}$ & Four biomass types & $\mathrm{N}$ & $\mathrm{Y}$ & $\mathrm{Y}$ & $\mathrm{Y}$ \\
\hline
\end{tabular}


Table 6. Cont.

\begin{tabular}{|c|c|c|c|c|c|c|c|c|c|c|}
\hline \multirow{2}{*}{ Ref. } & \multicolumn{5}{|l|}{ Fossil Fuel } & \multicolumn{5}{|l|}{ Biomass/Waste } \\
\hline & Description & 1 & 2 & 3 & 4 & Description & 1 & 2 & 3 & 4 \\
\hline [116] & Two coal types & M, S & $\mathrm{Y}$ & $\mathrm{Y}$ & $\mathrm{Y}$ & Olive waste & M, S & $\mathrm{Y}$ & $\mathrm{Y}$ & $\mathrm{Y}$ \\
\hline [117] & Two coal types & M, S & $\mathrm{Y}$ & $\mathrm{Y}$ & Y & Wood pellets & M, S & $\mathrm{Y}$ & Y & $\mathrm{Y}$ \\
\hline [118] & Turkish lignites & $\mathrm{M}$ & $\mathrm{Y}$ & $\mathrm{Y}$ & $\mathrm{Y}$ & Two waste biomass types & M, Sh & Y & $\mathrm{Y}$ & $\mathrm{Y}$ \\
\hline [68] & Coal & $\mathrm{N}$ & $\mathrm{Y}$ & $\mathrm{Y}$ & $\mathrm{Y}$ & Tobacco waste & $\mathrm{N}$ & $\mathrm{Y}$ & Y & $\mathrm{Y}$ \\
\hline [119] & Polish hard coal & $\mathrm{N}$ & $\mathrm{Y}$ & $\mathrm{Y}$ & $\mathrm{Y}$ & Alder wood chips & $\mathrm{N}$ & $\mathrm{Y}$ & $\mathrm{Y}$ & $\mathrm{Y}$ \\
\hline [57] & Coal Pittsburgh No. 8 & $\mathrm{~N}$ & $\mathrm{Y}$ & $\mathrm{Y}$ & $\mathrm{Y}$ & Wood & $\mathrm{N}$ & $\mathrm{Y}$ & $\mathrm{Y}$ & $\mathrm{Y}$ \\
\hline [120] & Coal and coal ash & M, S & $\mathrm{Y}$ & $\mathrm{N}$ & Y & Spruce + Miscanthus & M, S & Y & $\mathrm{N}$ & Y \\
\hline [31] & Multiple coal types, NG & $\mathrm{V}$ & $\mathrm{Y}$ & $\mathrm{Y}$ & $\mathrm{Y}$ & Multiple biomass types & $\mathrm{V}$ & $\mathrm{Y}$ & Y & $\mathrm{Y}$ \\
\hline [121] & Coal & $\mathrm{N}$ & $\mathrm{Y}$ & $\mathrm{Y}$ & Y & Straw, wood & $\mathrm{N}$ & Y & $\mathrm{Y}$ & $\mathrm{Y}$ \\
\hline [86] & Hard coal & M & $\mathrm{Y}$ & $\mathrm{Y}$ & $\mathrm{Y}$ & Straw, woody biomass & M & $\mathrm{Y}$ & $\mathrm{Y}$ & $\mathrm{Y}$ \\
\hline [122] & Two lignites & M & $\mathrm{Y}$ & $\mathrm{Y}$ & $\mathrm{Y}$ & Olive cake & M & Y & Y & Y \\
\hline [123] & Coal & M & $\mathrm{Y}$ & $\mathrm{Y}$ & $\mathrm{Y}$ & Biomass & M & $\mathrm{Y}$ & $\mathrm{Y}$ & $\mathrm{Y}$ \\
\hline [124] & Coal & M & $\mathrm{Y}$ & $\mathrm{Y}$ & $\mathrm{Y}$ & Biomass & M & $\mathrm{Y}$ & $\mathrm{Y}$ & $\mathrm{Y}$ \\
\hline [41] & Bituminous coal & $\mathrm{N}$ & $\mathrm{Y}$ & $\mathrm{Y}$ & $\mathrm{Y}$ & Three biomass types & $\mathrm{N}$ & $\mathrm{Y}$ & $\mathrm{Y}$ & $\mathrm{Y}$ \\
\hline [25] & Bituminous coal & M & $\mathrm{Y}$ & $\mathrm{Y}$ & $\mathrm{Y}$ & Corncob and hardwood & M & $\mathrm{Y}$ & $\mathrm{Y}$ & $\mathrm{Y}$ \\
\hline [38] & Bituminous coal & M, S & $\mathrm{Y}$ & $\mathrm{Y}$ & $\mathrm{Y}$ & Four woody biomasses & M, S & $\mathrm{Y}$ & $\mathrm{Y}$ & $\mathrm{Y}$ \\
\hline [49] & Two coals + LPG & M, S & $\mathrm{Y}$ & $\mathrm{Y}$ & $\mathrm{Y}$ & Woody biomass & M, S & $\mathrm{Y}$ & $\mathrm{Y}$ & $\mathrm{Y}$ \\
\hline [126] & Coal blends, NG & $\mathrm{M}$ & $\mathrm{Y}$ & $\mathrm{Y}$ & $\mathrm{Y}$ & Biomass & $\mathrm{M}$ & Y & $\mathrm{Y}$ & $\mathrm{Y}$ \\
\hline [58] & Two Coals & $\mathrm{M}$ & $\mathrm{Y}$ & $\mathrm{Y}$ & $\mathrm{Y}$ & Pine sawdust & M & $\mathrm{Y}$ & $\mathrm{Y}$ & Y \\
\hline [127] & Hard coal & $\mathrm{N}$ & $\mathrm{Y}$ & $\mathrm{Y}$ & $\mathrm{Y}$ & Various biomass types & $\mathrm{N}$ & $\mathrm{Y}$ & $\mathrm{Y}$ & Y \\
\hline [78] & Gas-flame coal & M & $\mathrm{Y}$ & $\mathrm{Y}$ & $\mathrm{Y}$ & Laying hens manure & M & $\mathrm{Y}$ & $\mathrm{Y}$ & Y \\
\hline [85] & Two lignites & $\mathrm{N}$ & $\mathrm{Y}$ & $\mathrm{Y}$ & $\mathrm{Y}$ & Red pine chips & $\mathrm{N}$ & $\mathrm{Y}$ & $\mathrm{Y}$ & $\mathrm{Y}$ \\
\hline [84] & Hard coal & $\mathrm{S}$ & $\mathrm{Y}$ & $\mathrm{Y}$ & $\mathrm{Y}$ & Torrefied biomass & $\mathrm{P}$ & $\mathrm{Y}$ & $\mathrm{Y}$ & $\mathrm{Y}$ \\
\hline [128] & Three coals & M & $\mathrm{Y}$ & $\mathrm{Y}$ & $\mathrm{N}$ & Straw & M & $\mathrm{Y}$ & $\mathrm{Y}$ & $\mathrm{N}$ \\
\hline [129] & Coal & $\mathrm{N}$ & $\mathrm{Y}$ & $\mathrm{Y}$ & $\mathrm{Y}$ & Sawdust & $\mathrm{N}$ & Y & $\mathrm{Y}$ & $\mathrm{y}$ \\
\hline [56] & Coal & $\mathrm{N}$ & $\mathrm{N}$ & $\mathrm{N}$ & $\mathrm{Y}$ & Poplar chips & $\mathrm{N}$ & $\mathrm{N}$ & $\mathrm{N}$ & Y \\
\hline [42] & Lignite & $\mathrm{N}$ & $\mathrm{Y}$ & $\mathrm{Y}$ & $\mathrm{Y}$ & Wood pellets & $\mathrm{N}$ & $\mathrm{Y}$ & Y & $\mathrm{Y}$ \\
\hline [20] & Lignite & M & $\mathrm{Y}$ & $\mathrm{Y}$ & $\mathrm{Y}$ & Biomass & M & $\mathrm{Y}$ & $\mathrm{Y}$ & $\mathrm{Y}$ \\
\hline [26] & Coal Illinois No. 6 & $\mathrm{~N}$ & $\mathrm{Y}$ & $\mathrm{Y}$ & $\mathrm{Y}$ & Avocado pits & M, S & $\mathrm{Y}$ & $\mathrm{Y}$ & $\mathrm{Y}$ \\
\hline [130] & Natural gas & $\mathrm{N}$ & $\mathrm{Y}$ & $\mathrm{Y}$ & $\mathrm{Y}$ & Biomass & $\mathrm{P}$ & $\mathrm{Y}$ & $\mathrm{Y}$ & $\mathrm{Y}$ \\
\hline [131] & Coal & M, S & $\mathrm{Y}$ & $\mathrm{Y}$ & $\mathrm{Y}$ & Various biomass types & M, S & $\mathrm{Y}$ & $\mathrm{Y}$ & $\mathrm{Y}$ \\
\hline [132] & Two coals & M, S & $\mathrm{Y}$ & $\mathrm{Y}$ & $\mathrm{Y}$ & Two biomass types, each raw or torrefied & M, S & $\mathrm{Y}$ & $\mathrm{Y}$ & $\mathrm{Y}$ \\
\hline [91] & Lignite & M, P & $\mathrm{Y}$ & $\mathrm{Y}$ & $\mathrm{Y}$ & Two biomass types, each raw or torrefied & M, P & $\mathrm{Y}$ & $\mathrm{Y}$ & $\mathrm{Y}$ \\
\hline [22] & Black coal & $\mathrm{M}, \mathrm{S}, \mathrm{P}$ & $\mathrm{Y}$ & $\mathrm{Y}$ & $\mathrm{Y}$ & Sewage sludge and Shiitake substrate & $\mathrm{M}, \mathrm{S}, \mathrm{P}$ & $\mathrm{Y}$ & $\mathrm{Y}$ & $\mathrm{Y}$ \\
\hline$[133]$ & Natural gas (methane) & $\mathrm{N}$ & $\mathrm{N}$ & $\mathrm{N}$ & $\mathrm{N}$ & Three simulated syngases & $\mathrm{N}$ & $\mathrm{Y}$ & $\mathrm{Y}$ & Y \\
\hline [134] & Anthracite & M, S & $\mathrm{Y}$ & $\mathrm{Y}$ & Y & RDF & M, S & Y & $\mathrm{Y}$ & $\mathrm{Y}$ \\
\hline$[71]$ & Coal water slurry, used turbine oil & $\mathrm{N}$ & $\mathrm{Y}$ & $\mathrm{Y}$ & $\mathrm{Y}$ & Various forest biomass types & M, S & $\mathrm{Y}$ & $\mathrm{Y}$ & $\mathrm{Y}$ \\
\hline [45] & Coal & M & $\mathrm{Y}$ & $\mathrm{Y}$ & Y & White wood pellets & $\mathrm{M}$ & $\mathrm{Y}$ & $\mathrm{Y}$ & Y \\
\hline$[50]$ & Indonesian low rank coal & M, S & $\mathrm{Y}$ & $\mathrm{Y}$ & $\mathrm{Y}$ & Sewage sludge, woody biomass & M, S & $\mathrm{Y}$ & $\mathrm{Y}$ & $\mathrm{Y}$ \\
\hline [135] & Czech brown coal & $\mathrm{N}$ & $\mathrm{Y}$ & $\mathrm{Y}$ & $\mathrm{Y}$ & Solid recovered fuel & $\mathrm{N}$ & $\mathrm{Y}$ & $\mathrm{Y}$ & $\mathrm{Y}$ \\
\hline [63] & Coal & M & $\mathrm{Y}$ & $\mathrm{Y}$ & $\mathrm{Y}$ & Biomass & M & $\mathrm{Y}$ & $\mathrm{Y}$ & $\mathrm{Y}$ \\
\hline$[136]$ & Australian bit coal + LPG & $\mathrm{N}$ & $\mathrm{Y}$ & $\mathrm{Y}$ & $\mathrm{Y}$ & Woody biomass & $\mathrm{N}$ & $\mathrm{Y}$ & $\mathrm{Y}$ & Y \\
\hline [23] & Low rank Bosnian coals & M & $\mathrm{Y}$ & $\mathrm{Y}$ & $\mathrm{Y}$ & Sawdust and Miscanthus & M & Y & $\mathrm{Y}$ & $\mathrm{Y}$ \\
\hline$[28]$ & Greek lignite and NG & M, S & $\mathrm{Y}$ & $\mathrm{Y}$ & $\mathrm{Y}$ & Biomass & M, S & Y & $\mathrm{Y}$ & $\mathrm{Y}$ \\
\hline [138] & Bituminous coal & $\mathrm{N}$ & $\mathrm{Y}$ & $\mathrm{Y}$ & $\mathrm{Y}$ & Composite biomass pellets & $\mathrm{P}$ & Y & $\mathrm{Y}$ & $\mathrm{Y}$ \\
\hline [70] & Coal & $\mathrm{N}$ & $\mathrm{N}$ & $\mathrm{N}$ & $\mathrm{N}$ & Palm oil production residual biomass & $\mathrm{P}$ & $\mathrm{N}$ & $\mathrm{N}$ & Y \\
\hline [139] & Coal & $\mathrm{M}$ & $\mathrm{Y}$ & $\mathrm{Y}$ & $\mathrm{N}$ & Biomass & $\mathrm{M}$ & $\mathrm{Y}$ & $\mathrm{Y}$ & $\mathrm{N}$ \\
\hline [47] & Three coals & M, S & $\mathrm{Y}$ & $\mathrm{Y}$ & $\mathrm{Y}$ & Two wastes & M, S & $\mathrm{Y}$ & $\mathrm{Y}$ & $\mathrm{Y}$ \\
\hline$[141]$ & Lignite & S & $\mathrm{Y}$ & $\mathrm{Y}$ & Y & Pine sawdust & $\mathrm{S}$ & Y & $\mathrm{Y}$ & $\mathrm{Y}$ \\
\hline [39] & Coal, coal slurry & $\mathrm{N}$ & $\mathrm{Y}$ & $\mathrm{Y}$ & Y & Sunflower pellets and woodchips & $\mathrm{N}$ & Y & $\mathrm{Y}$ & $\mathrm{Y}$ \\
\hline$[143]$ & Australian coal & $\mathrm{N}$ & $\mathrm{Y}$ & $\mathrm{Y}$ & $\mathrm{N}$ & Oat straw, gumwood & $\mathrm{N}$ & $\mathrm{Y}$ & $\mathrm{Y}$ & $\mathrm{N}$ \\
\hline [43] & Anthracite & M & $\mathrm{Y}$ & $\mathrm{Y}$ & Y & Sawmill sludge & M & Y & $\mathrm{Y}$ & $\mathrm{Y}$ \\
\hline [83] & Low rank coal & M, S & $\mathrm{Y}$ & $\mathrm{Y}$ & $\mathrm{Y}$ & Tobacco stalk & M, S & $\mathrm{Y}$ & $\mathrm{Y}$ & Y \\
\hline [144] & Coal & $\mathrm{N}$ & $\mathrm{Y}$ & $\mathrm{Y}$ & Y & Corn stalk & $\mathrm{N}$ & $\mathrm{Y}$ & $\mathrm{Y}$ & $\mathrm{Y}$ \\
\hline$[145]$ & Coal blends & $\mathrm{N}$ & $\mathrm{Y}$ & $\mathrm{Y}$ & $\mathrm{Y}$ & Multiple biomass types & $\mathrm{N}$ & $\mathrm{Y}$ & $\mathrm{Y}$ & $\mathrm{Y}$ \\
\hline [90] & Bituminous coal & $\mathrm{N}$ & $\mathrm{Y}$ & $\mathrm{Y}$ & Y & Soybean stalk & $\mathrm{N}$ & $\mathrm{Y}$ & $\mathrm{Y}$ & $\mathrm{Y}$ \\
\hline [46] & Industrial coal slurry & M & $\mathrm{Y}$ & $\mathrm{Y}$ & $\mathrm{Y}$ & Municipal sewage sludge & M & $\mathrm{Y}$ & $\mathrm{Y}$ & $\mathrm{Y}$ \\
\hline
\end{tabular}


Table 6. Cont.

\begin{tabular}{|c|c|c|c|c|c|c|c|c|c|c|}
\hline \multirow{2}{*}{ Ref. } & \multicolumn{5}{|c|}{ Fossil Fuel } & \multicolumn{5}{|l|}{ Biomass/Waste } \\
\hline & Description & 1 & 2 & 3 & 4 & Description & 1 & 2 & 3 & 4 \\
\hline [147] & Bituminous coal & M, S & $\mathrm{Y}$ & $\mathrm{Y}$ & $\mathrm{Y}$ & Empty palm fruit bunches, wood pellets & M, S & $\mathrm{Y}$ & $\mathrm{Y}$ & $\mathrm{Y}$ \\
\hline [148] & Bituminous coal & M, S & $\mathrm{Y}$ & $\mathrm{Y}$ & Y & Microalgae biomass & M, S & $\mathrm{Y}$ & $\mathrm{Y}$ & $\mathrm{Y}$ \\
\hline [88] & Two coals & M, S & $\mathrm{Y}$ & $\mathrm{Y}$ & $\mathrm{Y}$ & Sawmill wood waste, miscanthus & M, S & $\mathrm{Y}$ & $\mathrm{Y}$ & $\mathrm{Y}$ \\
\hline [24] & Two coals & M, S & $\mathrm{Y}$ & $\mathrm{Y}$ & $\mathrm{Y}$ & Wood pellets & M, S & $\mathrm{Y}$ & $\mathrm{Y}$ & $\mathrm{Y}$ \\
\hline [149] & Hard coal & $\mathrm{M}$ & $\mathrm{Y}$ & $\mathrm{Y}$ & $\mathrm{Y}$ & Pelletized sawdust & $\mathrm{P}, \mathrm{M}$ & $\mathrm{Y}$ & $\mathrm{Y}$ & $\mathrm{Y}$ \\
\hline [150] & anthracite & $\mathrm{S}$ & $\mathrm{Y}$ & $\mathrm{Y}$ & $\mathrm{N}$ & Three hydrochars & $S$ & $\mathrm{Y}$ & $\mathrm{Y}$ & $\mathrm{N}$ \\
\hline [29] & Coal & $\mathrm{N}$ & $\mathrm{Y}$ & $\mathrm{Y}$ & $\mathrm{N}$ & Wheat straw & $\mathrm{N}$ & Y & Y & $\mathrm{N}$ \\
\hline [59] & Coal & $\mathrm{N}$ & $\mathrm{Y}$ & $\mathrm{Y}$ & $\mathrm{Y}$ & Wheat straw & $\mathrm{N}$ & $\mathrm{Y}$ & $\mathrm{Y}$ & $\mathrm{Y}$ \\
\hline$[151]$ & NG (methane) & $\mathrm{N}$ & $\mathrm{N}$ & $\mathrm{N}$ & $\mathrm{N}$ & Waste wood & $\mathrm{N}$ & Y & $\mathrm{Y}$ & $\mathrm{Y}$ \\
\hline [152] & Bituminous coal & $\mathrm{N}$ & $\mathrm{Y}$ & $\mathrm{Y}$ & $\mathrm{Y}$ & Three biomass types & $\mathrm{N}$ & $\mathrm{Y}$ & $\mathrm{Y}$ & $\mathrm{Y}$ \\
\hline [21] & High rank bituminous coal & $\mathrm{N}$ & $\mathrm{Y}$ & $\mathrm{Y}$ & Y & White and black pellets & $\mathrm{N}$ & Y & $\mathrm{Y}$ & $\mathrm{y}$ \\
\hline [153] & Coal & $\mathrm{N}$ & $\mathrm{Y}$ & $\mathrm{Y}$ & $\mathrm{N}$ & Olive waste & $\mathrm{N}$ & $\mathrm{Y}$ & $\mathrm{Y}$ & $\mathrm{N}$ \\
\hline$[40]$ & Coal & M, S & $\mathrm{Y}$ & $\mathrm{Y}$ & $\mathrm{N}$ & Pine sawdust & M, S & $\mathrm{Y}$ & $\mathrm{Y}$ & $\mathrm{N}$ \\
\hline [154] & Lignite & M, S & $\mathrm{Y}$ & $\mathrm{Y}$ & $\mathrm{Y}$ & Poultry litter, raw and torrefied & M, S & $\mathrm{Y}$ & $\mathrm{Y}$ & $\mathrm{Y}$ \\
\hline [27] & Two low quality coals & $\mathrm{N}$ & $\mathrm{Y}$ & $\mathrm{Y}$ & $\mathrm{Y}$ & Two RDF types & $\mathrm{N}$ & $\mathrm{Y}$ & $\mathrm{Y}$ & $\mathrm{Y}$ \\
\hline [155] & Bituminous coal & $\mathrm{N}$ & $\mathrm{Y}$ & $\mathrm{Y}$ & $\mathrm{Y}$ & Four woody biomass types & $\mathrm{N}$ & $\mathrm{Y}$ & $\mathrm{Y}$ & $\mathrm{Y}$ \\
\hline$[48]$ & Slurry cake-waste coal & M, S & $\mathrm{Y}$ & $\mathrm{Y}$ & $\mathrm{Y}$ & MSW components and used turbine oil & M, S & $\mathrm{Y}$ & $\mathrm{Y}$ & $\mathrm{Y}$ \\
\hline [156] & Shale coal & $\mathrm{M}$ & $\mathrm{N}$ & $\mathrm{N}$ & Y & Three biomass types & M & $\mathrm{N}$ & $\mathrm{N}$ & $\mathrm{Y}$ \\
\hline [157] & Two coals & M & $\mathrm{Y}$ & $\mathrm{N}$ & $\mathrm{Y}$ & Biomass from phytoremediation & M & $\mathrm{Y}$ & $\mathrm{N}$ & $\mathrm{Y}$ \\
\hline [158] & Peat & $\mathrm{N}$ & $\mathrm{Y}$ & $\mathrm{Y}$ & $\mathrm{Y}$ & Wheat bran & $\mathrm{N}$ & $\mathrm{Y}$ & $\mathrm{Y}$ & $\mathrm{Y}$ \\
\hline
\end{tabular}

\section{Direct, Parallel, and Indirect (Gasification) Co-Combustion}

Available technologies offer several routes for biomass or waste and fossil fuel coutilization for heat and power production, while the application of each depends on many technical, economic, and environmental aspects [32,36]. They are altogether referred to as co-combustion $[10,36,74]$ :

- $\quad$ Direct co-combustion

- Parallel co-combustion

- Indirect co-combustion

Direct co-combustion is the most frequently applied option employing a single combustor with either common or separate feeds of fossil fuel and biomass or waste [89]. Both flue gas and solid residues are obtained as single material streams. Such solution requires the lowest specific investment cost compared to other co-combustion layouts [16,55]. Special applications of co-combustion, such as reburning or afterburning, are discussed in Section 8.

Parallel co-combustion employs separate combustors and the following heat transfer equipment for fossil fuel and biomass or waste [10]. Such a layout can be recommended for feedstock with low slagging and fouling propensity [103]. Separate ash streams are obtained, which may be an advantage if their properties differ substantially or the changeable biomass or waste composition poses a problem in direct co-firing. Table 7 documents the composition variability of fuel gas obtained from laboratory to full scale gasification and pyrolysis reactors operating with various feedstock and under various conditions. 
Table 7. Key parameters and composition of gas obtained from various gasification and pyrolysis reactors. Legend: RDF $=$ refuse derived fuel.

\begin{tabular}{|c|c|c|c|c|c|c|c|c|c|}
\hline Reference & [5] & [159] & [160] & [160] & [161] & [162] & [163] & [164] & [165] \\
\hline $\begin{array}{c}\text { Process, } \\
\text { Equipment }\end{array}$ & $\begin{array}{l}\text { Laboratory } \\
\text { Screw } \\
\text { Pyrolyzer }\end{array}$ & $\begin{array}{c}\text { Batch Fixed Bed Gasifier } \\
\text { + Secondary } \\
\text { Catalytic Reactor }\end{array}$ & $\begin{array}{l}\text { Fixed Bed } \\
\text { Pyrolyzer }\end{array}$ & $\begin{array}{c}\text { Auger } \\
\text { Pyrolyzer Pilot } \\
\text { Plant }\end{array}$ & \multicolumn{2}{|c|}{$\begin{array}{l}\text { Full Scale Dual } \\
\text { Fluid Bed Gasifier }\end{array}$} & $\begin{array}{l}\text { Laboratory Pyrolyzer- } \\
\text { TGA Apparatus }\end{array}$ & $\begin{array}{l}\text { Laboratory } \\
\text { Gasifier }\end{array}$ & $\begin{array}{l}\text { Laboratory } \\
\text { Gasifier }\end{array}$ \\
\hline Feedstock & Pine Needles & RDF & $\begin{array}{l}\text { Forestry } \\
\text { Waste }\end{array}$ & Waste Tires & $\begin{array}{l}\text { Woody } \\
\text { Biomass }\end{array}$ & $\begin{array}{l}\text { Wood } \\
\text { Pellets }\end{array}$ & Elephant Grass & $\begin{array}{l}\text { Waste Tires + } \\
\text { Plastics + } \\
\text { Biomass }\end{array}$ & $\begin{array}{l}\text { Wood } \\
\text { Pellets }\end{array}$ \\
\hline \multicolumn{7}{|c|}{ Gas Composition, \% vol. } & $\begin{array}{c}\text { Component Yield, \% wt. of } \\
\text { Dry Biomass }\end{array}$ & \multicolumn{2}{|c|}{ Gas Composition, \% vol. } \\
\hline $\mathrm{H}_{2}$ & 5.33 & 24.0 & 2.3 & 12.0 & 39.6 & 39.9 & 0 & 4.3 & 3.57 \\
\hline $\mathrm{N}_{2}$ & 56.41 & - & - & - & - & - & - & 56.7 & 67.51 \\
\hline $\mathrm{CO}$ & 15.89 & 20.0 & 43.9 & 20.0 & 22.8 & 24.0 & 6.6 & 5.6 & 15.3 \\
\hline $\mathrm{CO}_{2}$ & 12.00 & 10.1 & 41.8 & 8.0 & 20.9 & 25.2 & 18.6 & 7.9 & 10.8 \\
\hline $\mathrm{CH}_{4}$ & 9.22 & 2.7 & 9.6 & 7.4 & 9.8 & 8.6 & 0.6 & 3.7 & 2.82 \\
\hline $\mathrm{C}_{2} \mathrm{H}_{4}$ & 1.15 & 1.7 & 0.9 & 10.7 & 3.1 & 2.0 & 0.1 & 0.1 & - \\
\hline Other & - & 41.5 & 1.5 & 41.9 & 3.8 & 0.3 & 0.2 & - & - \\
\hline
\end{tabular}


Indirect co-combustion is realized by biomass or waste thermal treatment to obtain combustible gas (gasification) [166] or combustible gas, liquid, and solid fractions (pyrolysis) $[167,168]$. Such feedstock treatment requires higher specific investment cost compared to direct co-combustion $[169,170]$ but allows diverting (a part of) the obtained material for other use than heat and power production: chemicals and fuels production [166], or highly efficient heat and power production in combined cycles [171]; both requiring extensive gas cleaning [172]. The obtained combustible gas can be introduced to fossil fuels combustors as reburning fuel [110], which contributes to nitrogen oxides emissions reduction $[104,119,173]$.

\section{Basic Types of Combustors and Biomass Share}

Biomass and waste co-combustion with fossil fuels proceeds in combustors adapted from sole fossil fuel combustion to co-combustion. Stationary and moving grate combustors as well as fluidized bed boilers are applied for this purpose, each of them being suitable for biomass or waste with specific physical and chemical properties. Besides large and pilot scale combustors, laboratory scale equipment is widely used nowadays, often coupled with multi-instrumental data analysis to assess combustion behavior phenomena and their interaction in the co-combustion mode.

A review by Dai et al. (2008) [13] summed up basic features of the most often used combustors. Grate furnaces are characterized by their low specific investment cost, low operational costs, and a wide range of biomass types regarding their moisture, sulfur, or ash content to be co-combusted. However, their operation is constrained to low combustion temperatures due to corrosion risk due to ash melting at higher temperatures. Fuels with fine particles are unsuitable for such combustor type. Fluidized bed combustors can accommodate a wide variety of fuels, but the associated investment costs made it interesting for large scale systems only (above $40 \mathrm{MW}_{\mathrm{t}}$ ). They are inflexible regarding the fuel particle size and suffer from corrosion when firing high alkali fuels. Increased carbon content in ash was also observed. Pulverized fuel combustors are usually effective only for low moisture fuels, but low $\mathrm{NO}_{\mathrm{x}}$ emissions can be achieved with appropriate burners and high thermal efficiency due to low oxygen excess required.

Al-Mansour and Zuwala (2010) [32] stated that over 150 large scale co-combustion applications existed worldwide in 2007, and more than 220 in 2009, with more than 100 of them being in Europe. They analyzed experience from various co-combustion options: direct, indirect (gasification), and parallel co-combustion, the latter two being far less commercialized. The underlying technologies were evaluated via technical indices including $\mathrm{CO}_{2}$ emissions, applicability, operational experience, efficiency, process economics, and optimal biomass share, with grate co-combustion technology with the lowest potential and indirect co-combustion as the most promising technology, followed by direct co-combustion in FB boilers.

Agbor et al. (2014) [31] summed up various co-combustion techniques and concluded that CFB boilers are the most suitable for this purpose, followed by cyclone combustion that, however, accommodates lower biomass shares. Pulverized combustion on the other hand, can decrease $\mathrm{NO}_{\mathrm{x}}$ emissions significantly. Direct co-combustion in packed bed systems is problematic due to thermal efficiency loss and ash sintering tendency. Certain technical difficulties can be overcome with biomass pretreatment, such as pelletization and torrefaction, increasing biomass energy density increased and its macromolecular structure changed, or by application of a separate biomass feeding system accompanied with optimized combustion air staging.

Hein and Bemtgen (1998) [17] summarized the experience accumulated during two years from laboratory, pilot, and full scale co-combustion investigations from several European Union (EU) countries participating in a dedicated EU project. Several biomass sources were analyzed including dedicated woody crops, agricultural residues, and other biomass wastes co-combusted in a pulverized fuel (PF) and fluidized bed (FB) mode with biomass share of up to $40 \%$ wt. Evaluated results included trends in greenhouse 
gases (GHG) emissions and corrosion rates for different biomass shares in combusted fuel. The authors concluded that a positive effect both in nitrogen oxides $\left(\mathrm{NO}_{\mathrm{x}}\right)$ and sulfur oxides $\left(\mathrm{SO}_{\mathrm{x}}\right)$ emissions can be seen with $\mathrm{NO}_{\mathrm{x}}$ emissions decrease of up to $40 \%$ in full co-firing mode, which is strongly affected by air staging and the lack of major decrease in fuel conversion. Slagging and fouling tendencies needed to be investigated in long term experiments, especially with high chlorine and high alkaline biomass co-combustion.

Tillman (2000) [19] analyzed the outcomes of a United States of America (USA) wide program dedicated to coal and biomass co-combustion commercialization and compared them with results of similar programs in the EU. Biomass shares of up to $50 \%$ wt. were reported from full scale facilities in the USA. Experience from these facilities confirmed the conclusions of Hein and Bemtgen (1998) [17] in terms of $\mathrm{NO}_{x}$ emissions reduction exhibiting positive synergistic effect and a manageable decrease in boiler thermal efficiency of up to $2 \%$ in the co-combustion mode. The reported $\mathrm{NO}_{x}$ trend was further corroborated in a publication by Tillman (2015) [34] stating that the observed $\mathrm{NO}_{x}$ emission reduction was higher than what resulted from simple linear dependence assumption with varying biomass share in $65 \%$ of examined full scale systems. Moreover, a decrease of $\mathrm{CO}_{2}$ emissions of 2.7 to 3.15 ton per ton of combusted biomass was reported. Similarly to Tillman (2000) [19], Hughes (2000) [64] evaluated the underlying data from the USA facilities operating in the co-combustion mode and identified economics as the up-to-date major obstacle in further increase of biomass to power share.

Plant size (thermal input or electric power output) is a key factor determining the applied combustor type. Large plants (usually above $100 \mathrm{MW}_{\mathrm{t}}$ ) typically apply fluid combustors that offer excellent results both in terms of fuel burnout and produced greenhouse gases emissions $[13,32,36]$. Fuel particle size has to maintain within the desired interval, though. Such applications are best suitable for power production and cogeneration in thermal power plants as well as in large industrial and municipal heat and power plants and allow for biomass co-combustion ratio of above $20 \% \mathrm{wt}$. [13]. Pulverized fuel combustors are frequently applied in large scale applications where co-combustion proceeds either via a common system or in a separate fuel feeding system [19,31,89]. The first one requires adjustment of the existing fuel milling and delivery system and is typically suitable for biomass co-combustion ratio up to $5 \% \mathrm{wt}$. For the latter one, a complete independent biomass preparation and feeding system has to be constructed; however, the biomass co-combustion ratio can reach up to $20 \%$ wt. $[31,34]$.

For plant sizes of several $\mathrm{MW}_{\mathrm{t}}$ to several tens of $\mathrm{MW}_{\mathrm{t}}$, travelling grate furnaces and combustors are typical. They offer lower specific investment cost and lower operational costs in this size range than fluid combustors. They can fire a wide range of biomass types regarding their moisture, sulfur, or ash content. However, their operation is constrained to low combustion temperatures due to corrosion risk caused by ash melting at higher temperatures; they usually offer lower boiler thermal efficiency compared to fluid boilers or pulverized fuel boilers [31]. Fuels with fine particles are unsuitable for such combustor types. Grate combustors are often found in mid-size municipal heating plants. The majority of them have been refitted to co-combustion, sole biomass combustion, or to gas boilers.

Sub-MW $\mathrm{W}_{\mathrm{t}}$ and domestic boilers traditionally employ fixed bed combustion, which offers poorer performance in terms of fuel burnout and greenhouse gases emissions compared to fluid or traveling grate combustors, especially when firing low calorific value fuels. As an inefficient and environmentally unfriendly technology, it has been ruled out and replaced by internal gasification boilers.

Hot water boilers and utility steam boilers refitted for co-combustion generally show lower risk of high temperature corrosion than power plant steam boilers due to lower temperatures of the hottest heat exchange surfaces. Steam superheaters and reheaters in power plant boilers are the most critical elements with surface temperatures of around or above $500{ }^{\circ} \mathrm{C}$, which suits the deposition of alkali slags $[8,10,89]$. As the downgrade of steam parameters affects the power production efficiency, co-combustion of high chlorine 
and high alkaline biomass should be avoided in such installations, and the fuel slagging tendency should be managed with additives, as described in Section 7 in more detail.

Fuel premixing is another crucial factor affecting the combustor operation and efficiency in the co-combustion regime, especially in grate boilers and combustors. Lower density, larger share of volatiles, and faster biomass burnout in comparison with coal can lead to uneven fuel mix distribution over the grate and, subsequently, to formation of holes in the fuel layer after local biomass burnout. As a result, uneven air distribution can occur, followed by increased emissions and worsened fuel burnout on the rest of the grate.

Table 8 characterizes relevant papers from the biomass share and system performance point of view. The data overview corroborates the earlier findings by Tillman, Hughes, Hein, and Bemtgen that large scale systems employ biomass share usually less than $20 \%$ wt., whereas up to $100 \%$ biomass share applications were subject to computer simulations. Most studies used either boiler efficiency or specific fuel consumption as a straightforward energy performance indicator, while fewer of them analyzed the whole plant efficiency. Boiler efficiency (heat flux transferred to steam or hot water divided by total fuel energy input) reflects only energy losses associated with boiler operation change in the co-combustion regime, like fuel burnout, sootblowing steam consumption, and stack losses. It can be accepted as an objective criterion if produced steam parameters do not change in the co-combustion mode, or if the change in specific fuel consumption or that in internal power consumption is negligible. Plant efficiency (net plant power output divided by total fuel energy input) is influenced by more aspects than the boiler efficiency. Even if steam parameters in the co-combustion regime remain unchanged, the plant's internal steam or power consumption may differ from those in the original fossil fuel operation due to necessary adjustments in storage, transportation, and feedstock pretreatment. A downgrade in produced steam parameters results in lower plant efficiency due to lower steam specific work in the turbine, and it might even limit the maximal plant output. Thus, it can be concluded that while boiler efficiency can provide certain information about the system performance in the co-combustion regime, plant efficiency should be always evaluated and should always be preferred as an objective performance indicator.

Table 8. Characterization of relevant papers in terms of system layout performance indicators and biomass or waste share considered. Legend: $\mathrm{CFD}=$ computational fluid dynamics, $\mathrm{BE}=$ boiler efficiency, $\mathrm{PE}=$ plant efficiency, NPV = net present value, TIC $=$ total investment costs, $\mathrm{PI}=$ performance indicators, $\mathrm{N}=$ not analyzed, $\mathrm{CFB}=$ circulating fluidized bed, LCA = life cycle analysis.

\begin{tabular}{|c|c|c|c|}
\hline Ref. & Layout & Performance Indicator & Biomass Share, \% \\
\hline [37] & $140 \mathrm{t} / \mathrm{h}$ steam boiler & $\mathrm{BE}$ & $\leq 20$ thermal \\
\hline [33] & 18.7 $\mathrm{MW}_{\mathrm{e}}$ plant & specific fuel consumption & $\leq 60$ by mass \\
\hline$[13]$ & Multiple systems & $\mathrm{BE}$ & $\leq 100$ \\
\hline [114] & Simulated co-firing power plant & $\mathrm{BE}, \mathrm{PE}$ & $\leq 30$ by mass \\
\hline [115] & Spanish coal fired power plants & $\mathrm{BE}, \mathrm{PE}$ & $\leq 17$ by mass \\
\hline [119] & Biomass gasifier + gas reburning in flue gas of coal combustion & $\mathrm{BE}$ & $\leq 46$ thermal \\
\hline [57] & $500 \mathrm{MW}_{\mathrm{e}}$ coal boiler, CFD simulation & data from boiler simulation & $\leq 100$ \\
\hline$[85]$ & $0.75 \mathrm{MW}_{\mathrm{t}} \mathrm{CFB}$ combustor & $\mathrm{BE}$ & $\leq 23 \overline{b y}$ mass \\
\hline$[84]$ & $1 \mathrm{MW}_{\mathrm{t}} \mathrm{CFB}$ system with chemical looping for $\mathrm{CO}_{2}$ capture & Carbon capture efficiency & $\leq 50$ by mass \\
\hline [129] & $\begin{array}{c}1000 \mathrm{MW}_{\mathrm{e}} \text { supercritical oxyfuel power plant with CC modeled } \\
\text { in ChemCAD }\end{array}$ & net electric efficiency & $\leq 100$ \\
\hline [20] & Pulverized coal boiler, CFD calculations + Aspen Plus & $\mathrm{BE}$ & $\leq 100$ \\
\hline [130] & $\begin{array}{l}\text { Model calculation of energy consumption and } \mathrm{CO}_{2} \mathrm{LCA} \text { for } \\
\text { biomass co-firing }\end{array}$ & $\begin{array}{l}\text { energy consumption for } \\
\text { supply chain, } \\
\text { biomass pretreat }\end{array}$ & Not provided \\
\hline [132] & Laboratory drop tube furnace & combustion efficiency & $\leq 50$ by mass \\
\hline
\end{tabular}




\section{Slagging, Fouling, Corrosion, and Ash Properties}

High content of alkali and alkaline earth metals in biomass is a commonly acknowledged reason for higher fouling and slagging propensity of biomass and biomass-derived fuels compared to coal; this effect is accompanied with often reported corrosion in biomass and fossil fuel-biomass combustors [8,35]. Fly ash particles stick to boiler surface more eagerly if a liquid film is formed, which contributes to fouling. Simultaneously, increased chlorine and sulfur content in some biomass-derived fuels and wastes enhances the lowtemperature corrosion risk [36], while increased alkali content in fuels enhances slagging and high-temperature corrosion propensity. Alkali, alkaline earth metals, and sulfur entrainment in flue gas can lead to SCR catalyst deactivation. These factors negatively affect the plant efficiency and increase unplanned shutdown risk, but they also alter management costs. Table 9 documents numerous studies dealing with these aspects.

Stam and Brem (2019) [145] have recently analyzed the problem of boiler surface fouling during co-combustion of coal, wood, meat bone meal, and sheanut peels mixtures from the thermodynamic point of view to predict the $\mathrm{Na}-\mathrm{K}-\mathrm{SO}_{4}-\mathrm{Cl}$ salts eutectics occurrence and to assess the distribution of alkali metals in combustion products. Results indicated that suitable additives (e.g., ammonium sulfate) in suitable amounts significantly affect the presence of molten salts and therefore should be further examined with the prospect of boiler fouling suppression. A similar combined experimental and modeling approach was chosen by Sefidari et al. (2020) [21] for co-combustion impact assessment in an experimental iron ore pelletizing furnace, revealing that the complex co-combustion ash-iron ore dust system slagging tendency response to biomass share change varied significantly depending on actual operation conditions.

Zhang and Zhou (2019) [144] studied the effect of surface type on slagging characteristics during coal-corn co-combustion. They found that a nickel coated probe surface, compared with bare steel probe surface, exhibited weaker adhesion interactions with the slag formed, which resulted in repeated spontaneous slag shedding. Moreover, it slowed down the initial slag layer formation; both effects contribute to the increase of time-averaged heat flux through the nickel coated probe.

Opydo et al. (2019) [39] studied solids accumulation on various steel surfaces in a $400 \mathrm{MW}$ circulating fluidized bed boiler in the coal-biomass co-combustion mode and analyzed the accumulates. Three types of steel commonly used in boilers were tested with their samples placed inside the boiler directly after final steam superheater for $12 \mathrm{~h}$, where flue gas temperature reached around $850^{\circ} \mathrm{C}$. The front end section deposits were dense, sticking to the surfaces, and contained elevated amounts of sulfur and calcium as a result of calcium carbonate desulfurization method applied in the boiler. Alkali metals as well as aluminum and silica were found to accumulate preferentially in the rear section of the steel samples, with the rear deposits being less dense and consisting of fine ash and dust particles. No significant appearance or chemical composition differences were seen in the individual steel sample deposits.

Corrosive behavior of high alkaline biomass co-combustion flue gas was studied by Stephan et al. (2017) [128]. Straw and various coals were tested in a laboratory rig. High chromium content steel samples were placed in the combustor section for $24 \mathrm{~h}$, where the flue gases reached 700 to $900{ }^{\circ} \mathrm{C}$. Corrosion signal strength correlated with the straw share and chlorine content in the fuel, thus confirming earlier findings of Hein and Bemtgen (1998) [17]. On the contrary, Savolainen (2003) [106] did not detect any visible corrosion velocity increase in the full scale tests performed in a power plant boiler in the coal-sawdust co-combustion mode, most probably due to moderate biomass share in the fuel and much lower sawdust ash content compared to coals. Sondreal et al. (2001) [15] performed tests with various steel types to be used in future supercritical boilers under high sulfur-high ash content coal and high alkali biomass co-combustion conditions, finding that nickelchromium alloys withstand the corrosive environment for $1000 \mathrm{~h}$. Least affected was the alloy with highest chromium content, whereas those with chromium content below $20 \%$ wt. were affected to a much greater extent. Low temperature sulfur- and chlorine-based 
corrosion risk in co-combustion systems was investigated by Vainio et al. (2016) [174]. The system studied was a full scale bubbling fluidized bed boiler combusting bark, sludge, and solid recovered fuel. The results showed that sulfuric acid in flue gas in the vicinity of bag filters is minimal, which indicates efficient sulfur capture in ash; however, wet deposits rich in calcium and chlorine were observed on corrosion probes during long-term tests, which caused higher corrosion rates.

Physicochemical properties and chemical composition of ash obtained by co-combustion are directly related to co-combusted fuels, but are not additive measures [51]. Co-combustion ash can be characterized by bulk density, grains shape, loss on ignition, and melting temperature as the basic physicochemical indicators, and by chemical composition and metal content. Ash exhibits a very wide range of chemical composition due to varying biomass origin, as it is documented in Table 4 and data by Vassilev et al. $[175,176]$ with the range of $\mathrm{SiO}_{2}$ content in biomass ash from $<5$ to $>80 \%$ wt. and that of $\mathrm{CaO}$ from $<2$ to $>60 \%$ wt. Such composition variability affects all biomass ash and co-combustion ash properties, especially with higher biomass share in co-combustion processes.

Bulk density of co-combustion ashes was found to increase with the increasing biomass content, by a factor of up to four [51]. Ash grains shape depends mostly on the ignition conditions and the combustor type. Wang et al. (2008) [177] indicated that co-combustion ash grains are significantly smaller than those produced by biomass combustion only. Priyanto et al. (2016) [38] made the same conclusion when examining properties of ash produced by co-combustion of coal and various woody biomass in variable ratios.

Biomass and coal ash melting point was investigated by Vassilev et al. (2014) [178]. Especially herbaceous biomass ash and marine algae ash, rich in alkali metal oxides, exhibit significantly lower melting temperatures than coal ash, with minimal initial deformation temperature below $800{ }^{\circ} \mathrm{C}$ compared to over $1100{ }^{\circ} \mathrm{C}$ for all investigated coals. On the contrary, husks, pits, and woody biomass in general have comparable or even higher melting temperature than average coals due to increased content of high-melting constituents. Namkung et al. (2018) [50] studied the fusion behavior of ash of woody biomass and low rank coal and their blends. After being heated at $1000{ }^{\circ} \mathrm{C}$ for three hours, particles' structure and crystallinity of ashes were investigated by X-ray diffraction. The results corroborated the findings of Wang et al. [177], who observed ash particle size increase with the increasing biomass content in the blended fuel and confirmed the impact of phosphorus in blended ashes on their fusion behavior. Both Demirbas (2003) [89] and Dai et al. (2008) [13] concluded that the strength of biomass ash deposits is higher than that of coal combustion deposits only, and thus they are more difficult to remove. Priyanto et al. (2016) [38] recommended low ash content biomass to be co-combusted, preferably to reduce the ash fouling tendency. Hein and Bemtgen (1998) [17] summed up results from several full scale co-combustion trials, stating that at low biomass share, properties of the ash and fly ash formed resemble those from sole fossil fuel combustion, and though the slagging, fouling, and corrosion tendencies increased, they are at a tolerable level. More recently, results of low quality high ash lignite and herbaceous biomass co-combustion in a $500 \mathrm{~kW}$ combustor were presented by Fuller et al. (2018) [28], confirming only minor changes in the fly ash composition even at 50\% co-combustion biomass share. Kopzyński et al. (2017) [127] found that ash from torrefied biomass samples co-combusted with coal exhibits higher initial softening temperatures than that from raw biomass co-combustion.

Addition of cheap and widely available additives to modify ash properties is a research subject of numerous current studies. Considered additives are coal ash, kaolinite, or S-based additives $[35,139,145]$. Their addition aims at devolatilization of alkalis and supporting their chemical interactions with aluminosilicates. Protective coatings of hottest boiler heat exchanger surfaces are also applied [53]. Yao et al. (2019) [90] tested the effect of $\mathrm{K}_{2} \mathrm{CO}_{3}$ addition to biomass-coal blends on the ash fusion and agglomeration properties and mineralogy, finding that it enhanced ash agglomeration and modified its fusion temperatures. All these findings were corroborated by further research by Yao et al. (2020) [40] focused on coal-pine sawdust ashes properties and their modification by $\mathrm{K}_{2} \mathrm{CO}_{3}$ 
addition, an effective trap for volatile alkalis in the formed glassy phase. Stam and Brem (2019) [145] examined ammonia sulfate addition effect on possible $\mathrm{Na}-\mathrm{K}-\mathrm{SO}_{4}$-Cl eutectics formation. While the use of sulfur-containing additives seems to be contradicting the effort to suppress boiler corrosion, it has a beneficial effect on alkali and chlorine entrapment in ash, reducing thus the high temperature corrosion risk [10].

Jeong et al. (2019) [147] determined an optimal co-combustion ratio for coal with wood pellets and empty fruit bunches as 10 and $15 \%$ wt., respectively. Increasing the biomass cocombustion share up to these thresholds resulted in lower ash deposition due to overall ash amount decrease. Above the threshold values, however, biomass-origin ash agglomeration led to increased ash deposition. Kazagic et al. (2018) [23] investigated fouling and slagging properties of ash from different biomass and coal co-combustion ratio using Bosnian coals and woody biomass or miscanthus obtained in a laboratory temperature-controlled furnace. The results showed that while unburned carbon content in ash deposits was negligible in all fuel composition combinations, in slag collected at the bottom of the furnace the carbon content increased from $<1 \%$ wt., with biomass fraction in fuel increase from $15 \%$ to $25 \%$. Ash slagging and fouling tendencies remained low to medium in all investigated cases at the combustion temperature of $1250{ }^{\circ} \mathrm{C}$ but increased significantly in experiments with combustion temperature of $1450{ }^{\circ} \mathrm{C}$. Ash softening temperature was estimated in the range of 1200 to $1300^{\circ} \mathrm{C}$ for all ash samples.

Table 9. Characterization of papers' relevance to operational and process aspects. Legend: VS—variable biomass/waste share $(\mathrm{Y}=$ Yes, $\mathrm{N}=\mathrm{No}), \mathrm{OC}=$ oxycombustion applied $(\mathrm{Y}=\mathrm{Yes}, \mathrm{N}=\mathrm{No}), \mathrm{AS}=$ Air staging applied $(\mathrm{Y}=\mathrm{Yes}, \mathrm{N}=\mathrm{No})$, $\mathrm{REB}=$ reburning applied $(\mathrm{Y}=$ Yes, $\mathrm{N}=\mathrm{No}), \mathrm{AE}=$ Variable air/oxygen excess applied $(\mathrm{Y}=\mathrm{Yes}, \mathrm{N}=\mathrm{No}), 1-\mathrm{fouling}$, 2-slagging, 3-corrosion, 4-fuel burnout, 5-ignition temperature, 6-kinetic parameters of combustion, 7-temperature profile/field, 8-chemical composition, 9-physical properties and utilization, Other $=$ other aspects $(\mathrm{Y}=$ investigated, $\mathrm{N}=$ not investigated).

\begin{tabular}{|c|c|c|c|c|c|c|c|c|c|c|c|c|c|c|c|}
\hline \multirow{2}{*}{ Ref. } & \multirow{2}{*}{ VS } & \multicolumn{4}{|c|}{ Combustion } & \multicolumn{8}{|c|}{ Process Aspects } & \multicolumn{2}{|c|}{ Ash } \\
\hline & & OC & AS & REB & $\mathrm{AE}$ & 1 & 2 & 3 & 4 & 5 & 6 & 7 & Other & 8 & 9 \\
\hline$[18]$ & Y & $\mathrm{N}$ & $\mathrm{N}$ & $\mathrm{N}$ & $\mathrm{N}$ & $\mathrm{N}$ & $\mathrm{N}$ & $\mathrm{N}$ & $\mathrm{Y}$ & $\mathrm{N}$ & $\mathrm{N}$ & $\mathrm{N}$ & $\mathrm{Y}$ & $\mathrm{Y}$ & $\mathrm{Y}$ \\
\hline [17] & Y & $\mathrm{N}$ & Y & $\mathrm{N}$ & $\mathrm{N}$ & $\mathrm{Y}$ & $\mathrm{Y}$ & $\mathrm{Y}$ & $\mathrm{Y}$ & $\mathrm{N}$ & $\mathrm{N}$ & $\mathrm{N}$ & $\mathrm{N}$ & $\mathrm{N}$ & $\mathrm{N}$ \\
\hline [104] & $\mathrm{Y}$ & $\mathrm{N}$ & $\mathrm{Y}$ & $\mathrm{Y}$ & $\mathrm{Y}$ & $\mathrm{N}$ & $\mathrm{N}$ & $\mathrm{N}$ & $\mathrm{N}$ & $\mathrm{N}$ & $\mathrm{N}$ & $\mathrm{N}$ & $\mathrm{Y}$ & $\mathrm{N}$ & $\mathrm{N}$ \\
\hline [19] & Y & $\mathrm{N}$ & $\mathrm{N}$ & $\mathrm{N}$ & $\mathrm{N}$ & $\mathrm{Y}$ & $\mathrm{N}$ & $\mathrm{N}$ & $\mathrm{N}$ & $\mathrm{N}$ & $\mathrm{N}$ & $\mathrm{N}$ & $\mathrm{Y}$ & $\mathrm{Y}$ & $\mathrm{Y}$ \\
\hline [105] & Y & $\mathrm{N}$ & Y & $\mathrm{N}$ & $\mathrm{N}$ & $\mathrm{Y}$ & $\mathrm{Y}$ & $\mathrm{Y}$ & $\mathrm{Y}$ & $\mathrm{N}$ & $\mathrm{N}$ & $\mathrm{N}$ & Y & $\mathrm{Y}$ & $\mathrm{Y}$ \\
\hline [8] & Y & $\mathrm{N}$ & $\mathrm{N}$ & $\mathrm{N}$ & $\mathrm{N}$ & $\mathrm{Y}$ & $\mathrm{Y}$ & $\mathrm{Y}$ & $\mathrm{Y}$ & $\mathrm{Y}$ & $\mathrm{Y}$ & $\mathrm{Y}$ & $\mathrm{Y}$ & $\mathrm{Y}$ & $\mathrm{Y}$ \\
\hline [44] & Y & $\mathrm{N}$ & Y & $\mathrm{N}$ & $\mathrm{N}$ & $\mathrm{Y}$ & $\mathrm{N}$ & $\mathrm{N}$ & $\mathrm{Y}$ & $\mathrm{N}$ & $\mathrm{N}$ & $\mathrm{N}$ & $\mathrm{N}$ & $\mathrm{Y}$ & $\mathrm{N}$ \\
\hline [82] & $Y$ & $\mathrm{~N}$ & $\mathrm{~N}$ & $\mathrm{~N}$ & $\mathrm{Y}$ & $\mathrm{N}$ & $\mathrm{N}$ & $\mathrm{N}$ & $\mathrm{Y}$ & $\mathrm{N}$ & $\mathrm{N}$ & $\mathrm{Y}$ & Y & $\mathrm{Y}$ & $\mathrm{N}$ \\
\hline [106] & Y & $\mathrm{N}$ & Y & $\mathrm{N}$ & $\mathrm{N}$ & $\mathrm{Y}$ & $\mathrm{Y}$ & $\mathrm{N}$ & $\mathrm{Y}$ & $\mathrm{N}$ & $\mathrm{N}$ & $\mathrm{N}$ & $\mathrm{Y}$ & $\mathrm{Y}$ & $\mathrm{Y}$ \\
\hline [35] & $\mathrm{N}$ & $\mathrm{N}$ & $\mathrm{N}$ & $\mathrm{N}$ & $\mathrm{N}$ & $\mathrm{Y}$ & $\mathrm{N}$ & $\mathrm{Y}$ & $\mathrm{N}$ & $\mathrm{N}$ & $\mathrm{N}$ & $\mathrm{Y}$ & $\mathrm{Y}$ & $\mathrm{N}$ & $\mathrm{Y}$ \\
\hline [107] & $\mathrm{N}$ & $\mathrm{N}$ & $\mathrm{N}$ & $\mathrm{N}$ & $\mathrm{N}$ & $\mathrm{N}$ & $\mathrm{N}$ & $\mathrm{N}$ & $\mathrm{Y}$ & $\mathrm{N}$ & $\mathrm{Y}$ & $\mathrm{Y}$ & $\mathrm{N}$ & $\mathrm{N}$ & $\mathrm{N}$ \\
\hline [37] & $Y$ & $\mathrm{~N}$ & $\mathrm{~N}$ & $\mathrm{~N}$ & $\mathrm{~N}$ & $\mathrm{Y}$ & $\mathrm{N}$ & $\mathrm{N}$ & $\mathrm{N}$ & $\mathrm{N}$ & $\mathrm{N}$ & $\mathrm{N}$ & $\mathrm{N}$ & $\mathrm{Y}$ & $\mathrm{N}$ \\
\hline [108] & Y & $\mathrm{N}$ & $\mathrm{N}$ & $\mathrm{N}$ & $\mathrm{N}$ & $\mathrm{N}$ & $\mathrm{N}$ & $\mathrm{N}$ & $\mathrm{Y}$ & $\mathrm{N}$ & $\mathrm{N}$ & $\mathrm{Y}$ & $\mathrm{Y}$ & $\mathrm{N}$ & $\mathrm{N}$ \\
\hline [36] & $\mathrm{Y}$ & $\mathrm{N}$ & $\mathrm{N}$ & $\mathrm{N}$ & $\mathrm{N}$ & $\mathrm{Y}$ & $\mathrm{N}$ & $\mathrm{N}$ & $\mathrm{N}$ & $\mathrm{N}$ & $\mathrm{N}$ & $\mathrm{N}$ & Y & $\mathrm{Y}$ & $\mathrm{N}$ \\
\hline [33] & Y & $\mathrm{N}$ & $\mathrm{Y}$ & $\mathrm{N}$ & $\mathrm{N}$ & $\mathrm{N}$ & $\mathrm{N}$ & $\mathrm{N}$ & $\mathrm{N}$ & $\mathrm{N}$ & $\mathrm{N}$ & $\mathrm{N}$ & $\mathrm{N}$ & $\mathrm{N}$ & $\mathrm{N}$ \\
\hline [13] & Y & $\mathrm{N}$ & $\mathrm{N}$ & $\mathrm{N}$ & $\mathrm{N}$ & $\mathrm{Y}$ & $\mathrm{Y}$ & $\mathrm{Y}$ & $\mathrm{Y}$ & $\mathrm{N}$ & $\mathrm{N}$ & $\mathrm{N}$ & $\mathrm{Y}$ & $\mathrm{Y}$ & $\mathrm{Y}$ \\
\hline [109] & Y & $\mathrm{Y}$ & $\mathrm{N}$ & $\mathrm{N}$ & $\mathrm{N}$ & $\mathrm{N}$ & $\mathrm{N}$ & $\mathrm{N}$ & $\mathrm{Y}$ & $\mathrm{Y}$ & $\mathrm{N}$ & $\mathrm{N}$ & $\mathrm{Y}$ & $\mathrm{N}$ & $\mathrm{N}$ \\
\hline [32] & Y & $\mathrm{N}$ & $\mathrm{N}$ & $\mathrm{N}$ & $\mathrm{N}$ & $\mathrm{Y}$ & $\mathrm{Y}$ & $\mathrm{N}$ & $\mathrm{N}$ & $\mathrm{N}$ & $\mathrm{N}$ & $\mathrm{N}$ & $\mathrm{N}$ & $\mathrm{N}$ & $\mathrm{N}$ \\
\hline [110] & Y & $\mathrm{N}$ & $\mathrm{N}$ & $\mathrm{Y}$ & $\mathrm{Y}$ & $\mathrm{N}$ & $\mathrm{N}$ & $\mathrm{N}$ & $\mathrm{N}$ & $\mathrm{N}$ & $\mathrm{N}$ & $\mathrm{N}$ & $\mathrm{Y}$ & $\mathrm{N}$ & $\mathrm{N}$ \\
\hline [111] & Y & $\mathrm{N}$ & $\mathrm{N}$ & $\mathrm{Y}$ & $\mathrm{N}$ & $\mathrm{N}$ & $\mathrm{N}$ & $\mathrm{N}$ & $\mathrm{Y}$ & $\mathrm{N}$ & $\mathrm{N}$ & $\mathrm{N}$ & $\mathrm{Y}$ & $\mathrm{N}$ & $\mathrm{N}$ \\
\hline [51] & $Y$ & $\mathrm{~N}$ & $\mathrm{~N}$ & $\mathrm{~N}$ & $\mathrm{~N}$ & $\mathrm{Y}$ & $\mathrm{Y}$ & $Y$ & $Y$ & $\mathrm{~N}$ & $\mathrm{~N}$ & $\mathrm{~N}$ & $\mathrm{~N}$ & $\mathrm{Y}$ & $Y$ \\
\hline [112] & Y & $\mathrm{N}$ & $\mathrm{N}$ & Y & Y & $\mathrm{N}$ & $\mathrm{N}$ & $\mathrm{N}$ & $\mathrm{N}$ & $\mathrm{N}$ & $\mathrm{N}$ & $\mathrm{N}$ & $\mathrm{Y}$ & $\mathrm{N}$ & $\mathrm{N}$ \\
\hline [114] & Y & $\mathrm{N}$ & $\mathrm{N}$ & $\mathrm{N}$ & $\mathrm{N}$ & $\mathrm{N}$ & $\mathrm{N}$ & $\mathrm{N}$ & $\mathrm{N}$ & $\mathrm{N}$ & $\mathrm{N}$ & $\mathrm{N}$ & $\mathrm{N}$ & $\mathrm{Y}$ & $\mathrm{N}$ \\
\hline [115] & Y & $\mathrm{N}$ & $\mathrm{N}$ & $\mathrm{N}$ & $\mathrm{Y}$ & $\mathrm{N}$ & $\mathrm{N}$ & $\mathrm{N}$ & $\mathrm{N}$ & $\mathrm{N}$ & $\mathrm{N}$ & $\mathrm{Y}$ & $\mathrm{N}$ & $\mathrm{Y}$ & $\mathrm{N}$ \\
\hline [116] & Y & Y & $\mathrm{N}$ & $\mathrm{N}$ & $\mathrm{N}$ & $\mathrm{N}$ & $\mathrm{N}$ & $\mathrm{N}$ & $Y$ & $\mathrm{Y}$ & $\mathrm{N}$ & $\mathrm{N}$ & $\mathrm{Y}$ & $\mathrm{N}$ & $\mathrm{N}$ \\
\hline [117] & Y & $\mathrm{N}$ & $\mathrm{N}$ & $\mathrm{N}$ & $\mathrm{N}$ & $\mathrm{N}$ & $\mathrm{N}$ & $\mathrm{N}$ & $\mathrm{N}$ & $\mathrm{Y}$ & $\mathrm{N}$ & $\mathrm{Y}$ & $\mathrm{N}$ & $\mathrm{N}$ & $\mathrm{N}$ \\
\hline
\end{tabular}


Table 9. Cont.

\begin{tabular}{|c|c|c|c|c|c|c|c|c|c|c|c|c|c|c|c|}
\hline \multirow{2}{*}{ Ref. } & \multirow{2}{*}{ VS } & \multicolumn{4}{|c|}{ Combustion } & \multicolumn{8}{|c|}{ Process Aspects } & \multicolumn{2}{|c|}{ Ash } \\
\hline & & OC & AS & REB & $\mathrm{AE}$ & 1 & 2 & 3 & 4 & 5 & 6 & 7 & Other & 8 & 9 \\
\hline [118] & $\mathrm{Y}$ & $\mathrm{Y}$ & $\mathrm{N}$ & $\mathrm{N}$ & $\mathrm{N}$ & $\mathrm{N}$ & $\mathrm{N}$ & $\mathrm{N}$ & $\mathrm{Y}$ & $\mathrm{Y}$ & $\mathrm{N}$ & $\mathrm{N}$ & $\mathrm{N}$ & $\mathrm{N}$ & $\mathrm{N}$ \\
\hline [119] & $\mathrm{N}$ & $\mathrm{N}$ & $\mathrm{N}$ & $\mathrm{Y}$ & $\mathrm{Y}$ & $\mathrm{N}$ & $\mathrm{N}$ & $\mathrm{N}$ & $\mathrm{N}$ & $\mathrm{N}$ & $\mathrm{N}$ & $\mathrm{N}$ & $\mathrm{N}$ & $\mathrm{N}$ & $\mathrm{N}$ \\
\hline [57] & $\mathrm{N}$ & $\mathrm{Y}$ & $\mathrm{N}$ & $\mathrm{N}$ & $\mathrm{N}$ & $\mathrm{N}$ & $\mathrm{N}$ & $\mathrm{N}$ & $\mathrm{N}$ & $\mathrm{N}$ & $\mathrm{N}$ & $\mathrm{Y}$ & $\mathrm{Y}$ & $\mathrm{N}$ & $\mathrm{Y}$ \\
\hline [120] & Y & $\mathrm{N}$ & $\mathrm{Y}$ & $\mathrm{N}$ & $\mathrm{N}$ & $\mathrm{Y}$ & Y & $\mathrm{N}$ & $\mathrm{Y}$ & $\mathrm{N}$ & $\mathrm{N}$ & $\mathrm{Y}$ & $\mathrm{Y}$ & $\mathrm{Y}$ & $\mathrm{Y}$ \\
\hline [31] & $\mathrm{Y}$ & $\mathrm{N}$ & Y & $\mathrm{Y}$ & $\mathrm{N}$ & $\mathrm{Y}$ & $\mathrm{Y}$ & $\mathrm{N}$ & $\mathrm{N}$ & $\mathrm{N}$ & $\mathrm{N}$ & $\mathrm{N}$ & $\mathrm{N}$ & $\mathrm{N}$ & $\mathrm{N}$ \\
\hline [121] & $\mathrm{Y}$ & $\mathrm{N}$ & Y & $\mathrm{N}$ & $\mathrm{N}$ & $\mathrm{N}$ & $\mathrm{N}$ & $\mathrm{N}$ & $\mathrm{Y}$ & $\mathrm{N}$ & $\mathrm{N}$ & $\mathrm{N}$ & $\mathrm{N}$ & $\mathrm{N}$ & $\mathrm{N}$ \\
\hline [11] & $\mathrm{N}$ & $\mathrm{N}$ & $\mathrm{N}$ & $\mathrm{N}$ & $\mathrm{N}$ & $\mathrm{N}$ & $\mathrm{N}$ & $\mathrm{N}$ & $\mathrm{N}$ & $\mathrm{N}$ & $\mathrm{N}$ & $\mathrm{N}$ & $\mathrm{N}$ & $\mathrm{Y}$ & $\mathrm{Y}$ \\
\hline [10] & $\mathrm{Y}$ & $\mathrm{N}$ & $\mathrm{N}$ & $\mathrm{N}$ & $\mathrm{N}$ & $\mathrm{Y}$ & $\mathrm{Y}$ & $\mathrm{Y}$ & $\mathrm{N}$ & $\mathrm{N}$ & $\mathrm{N}$ & $\mathrm{N}$ & $\mathrm{Y}$ & $\mathrm{N}$ & Y \\
\hline [86] & $\mathrm{Y}$ & $\mathrm{Y}$ & $\mathrm{N}$ & $\mathrm{N}$ & $\mathrm{Y}$ & $\mathrm{Y}$ & $\mathrm{Y}$ & $\mathrm{N}$ & $\mathrm{Y}$ & $\mathrm{N}$ & $\mathrm{N}$ & $\mathrm{N}$ & Y & $\mathrm{Y}$ & $\mathrm{N}$ \\
\hline [122] & $\mathrm{N}$ & $\mathrm{N}$ & $\mathrm{N}$ & $\mathrm{N}$ & $\mathrm{Y}$ & $\mathrm{N}$ & $\mathrm{N}$ & $\mathrm{N}$ & $\mathrm{N}$ & $\mathrm{N}$ & $\mathrm{N}$ & $\mathrm{N}$ & $\mathrm{Y}$ & $\mathrm{Y}$ & $\mathrm{Y}$ \\
\hline [123] & $\mathrm{Y}$ & $\mathrm{Y}$ & $Y$ & $\mathrm{~N}$ & $\mathrm{~N}$ & $\mathrm{~N}$ & $\mathrm{~N}$ & $\mathrm{~N}$ & $\mathrm{Y}$ & $\mathrm{N}$ & $\mathrm{N}$ & $\mathrm{Y}$ & $\mathrm{Y}$ & $\mathrm{N}$ & $\mathrm{N}$ \\
\hline [124] & $\mathrm{Y}$ & $\mathrm{Y}$ & $\mathrm{N}$ & $\mathrm{N}$ & $\mathrm{N}$ & $\mathrm{N}$ & $\mathrm{N}$ & $\mathrm{N}$ & $\mathrm{N}$ & $\mathrm{N}$ & $\mathrm{N}$ & $\mathrm{Y}$ & $\mathrm{Y}$ & $\mathrm{N}$ & $\mathrm{N}$ \\
\hline [41] & $\mathrm{Y}$ & $\mathrm{Y}$ & $\mathrm{N}$ & $\mathrm{N}$ & $\mathrm{N}$ & $\mathrm{N}$ & $\mathrm{N}$ & $\mathrm{N}$ & $\mathrm{N}$ & $\mathrm{N}$ & $\mathrm{N}$ & $\mathrm{N}$ & $\mathrm{Y}$ & $\mathrm{N}$ & $\mathrm{N}$ \\
\hline [125] & Y & $\mathrm{N}$ & $\mathrm{N}$ & $\mathrm{N}$ & $\mathrm{Y}$ & $\mathrm{N}$ & $\mathrm{N}$ & $\mathrm{N}$ & $\mathrm{N}$ & $\mathrm{N}$ & $\mathrm{N}$ & $\mathrm{Y}$ & Y & $\mathrm{N}$ & $\mathrm{N}$ \\
\hline [25] & Y & $\mathrm{Y}$ & $\mathrm{N}$ & $\mathrm{N}$ & $\mathrm{N}$ & $\mathrm{N}$ & $\mathrm{N}$ & $\mathrm{N}$ & $\mathrm{N}$ & $\mathrm{Y}$ & $\mathrm{N}$ & $\mathrm{N}$ & $\mathrm{Y}$ & $\mathrm{N}$ & $\mathrm{Y}$ \\
\hline [38] & $\mathrm{Y}$ & $\mathrm{N}$ & $\mathrm{N}$ & $\mathrm{N}$ & $\mathrm{N}$ & $\mathrm{N}$ & $\mathrm{Y}$ & $\mathrm{N}$ & $\mathrm{N}$ & $\mathrm{N}$ & $\mathrm{N}$ & $\mathrm{N}$ & $\mathrm{Y}$ & $\mathrm{Y}$ & Y \\
\hline [49] & $\mathrm{Y}$ & $\mathrm{N}$ & $\mathrm{Y}$ & $\mathrm{N}$ & $\mathrm{N}$ & $\mathrm{N}$ & $\mathrm{N}$ & $\mathrm{N}$ & $\mathrm{Y}$ & $\mathrm{N}$ & $\mathrm{N}$ & $\mathrm{Y}$ & $\mathrm{N}$ & $\mathrm{N}$ & $\mathrm{N}$ \\
\hline [126] & $\mathrm{Y}$ & $\mathrm{N}$ & $\mathrm{Y}$ & $\mathrm{Y}$ & $\mathrm{Y}$ & $\mathrm{N}$ & $\mathrm{N}$ & $\mathrm{N}$ & $\mathrm{N}$ & $\mathrm{N}$ & $\mathrm{N}$ & $\mathrm{N}$ & $\mathrm{Y}$ & $\mathrm{Y}$ & $\mathrm{N}$ \\
\hline [58] & $\mathrm{N}$ & $\mathrm{N}$ & $\mathrm{N}$ & $\mathrm{N}$ & $\mathrm{N}$ & $\mathrm{N}$ & $\mathrm{N}$ & $\mathrm{N}$ & $\mathrm{N}$ & $\mathrm{N}$ & $\mathrm{Y}$ & $\mathrm{Y}$ & Y & $\mathrm{N}$ & $\mathrm{N}$ \\
\hline [127] & $\mathrm{N}$ & $\mathrm{N}$ & $\mathrm{N}$ & $\mathrm{N}$ & $\mathrm{N}$ & $\mathrm{Y}$ & $\mathrm{Y}$ & $\mathrm{N}$ & $\mathrm{N}$ & $\mathrm{N}$ & $\mathrm{N}$ & $\mathrm{Y}$ & $\mathrm{N}$ & $\mathrm{Y}$ & Y \\
\hline [103] & $\mathrm{N}$ & $\mathrm{N}$ & $\mathrm{N}$ & $\mathrm{N}$ & $\mathrm{N}$ & $\mathrm{Y}$ & $\mathrm{Y}$ & $\mathrm{N}$ & $\mathrm{N}$ & $\mathrm{N}$ & $\mathrm{N}$ & $\mathrm{N}$ & $\mathrm{N}$ & $\mathrm{N}$ & $\mathrm{N}$ \\
\hline [78] & $\mathrm{Y}$ & $\mathrm{N}$ & $\mathrm{N}$ & $\mathrm{N}$ & $\mathrm{N}$ & $\mathrm{N}$ & $\mathrm{N}$ & $\mathrm{N}$ & $\mathrm{N}$ & $\mathrm{Y}$ & $\mathrm{Y}$ & $\mathrm{N}$ & $\mathrm{N}$ & $\mathrm{N}$ & $\mathrm{N}$ \\
\hline [85] & $\mathrm{N}$ & $\mathrm{N}$ & $\mathrm{N}$ & $\mathrm{N}$ & $\mathrm{Y}$ & $\mathrm{N}$ & $\mathrm{N}$ & $\mathrm{N}$ & $\mathrm{Y}$ & $\mathrm{N}$ & $\mathrm{N}$ & $\mathrm{Y}$ & $\mathrm{Y}$ & $\mathrm{Y}$ & $\mathrm{N}$ \\
\hline [84] & $\mathrm{N}$ & $\mathrm{N}$ & $\mathrm{N}$ & $\mathrm{N}$ & $\mathrm{N}$ & $\mathrm{N}$ & $\mathrm{N}$ & $\mathrm{N}$ & $\mathrm{N}$ & $\mathrm{N}$ & $\mathrm{N}$ & $\mathrm{N}$ & $\mathrm{Y}$ & $\mathrm{N}$ & $\mathrm{N}$ \\
\hline [128] & $\mathrm{Y}$ & $\mathrm{N}$ & $\mathrm{N}$ & $\mathrm{N}$ & $\mathrm{Y}$ & $\mathrm{N}$ & $\mathrm{N}$ & $\mathrm{Y}$ & $\mathrm{N}$ & $\mathrm{N}$ & $\mathrm{N}$ & $\mathrm{N}$ & $\mathrm{N}$ & $\mathrm{Y}$ & $\mathrm{N}$ \\
\hline [129] & $\mathrm{Y}$ & $\mathrm{Y}$ & $\mathrm{Y}$ & $\mathrm{N}$ & $\mathrm{Y}$ & $\mathrm{N}$ & $\mathrm{N}$ & $\mathrm{N}$ & $\mathrm{N}$ & $\mathrm{N}$ & $\mathrm{N}$ & $\mathrm{N}$ & $\mathrm{Y}$ & $\mathrm{N}$ & $\mathrm{N}$ \\
\hline [42] & $\mathrm{Y}$ & $\mathrm{Y}$ & $\mathrm{N}$ & $\mathrm{N}$ & $\mathrm{Y}$ & $\mathrm{N}$ & $\mathrm{N}$ & $\mathrm{N}$ & $\mathrm{N}$ & $\mathrm{N}$ & $\mathrm{N}$ & $\mathrm{N}$ & $\mathrm{Y}$ & $\mathrm{Y}$ & $\mathrm{Y}$ \\
\hline [20] & $\mathrm{N}$ & $\mathrm{N}$ & $\mathrm{Y}$ & $\mathrm{N}$ & $\mathrm{N}$ & $\mathrm{N}$ & $\mathrm{N}$ & $\mathrm{N}$ & $\mathrm{Y}$ & $\mathrm{N}$ & $\mathrm{N}$ & $Y$ & $\mathrm{Y}$ & $\mathrm{N}$ & $\mathrm{N}$ \\
\hline [26] & $\mathrm{Y}$ & $\mathrm{N}$ & $\mathrm{N}$ & $\mathrm{N}$ & $\mathrm{N}$ & $\mathrm{N}$ & $\mathrm{N}$ & $\mathrm{N}$ & $\mathrm{N}$ & $\mathrm{Y}$ & $\mathrm{Y}$ & $\mathrm{N}$ & $\mathrm{Y}$ & $\mathrm{N}$ & $\mathrm{N}$ \\
\hline [131] & $\mathrm{N}$ & $\mathrm{N}$ & $\mathrm{N}$ & $\mathrm{N}$ & $\mathrm{N}$ & $\mathrm{N}$ & $\mathrm{N}$ & $\mathrm{N}$ & $\mathrm{N}$ & $\mathrm{N}$ & $\mathrm{N}$ & $\mathrm{Y}$ & $\mathrm{Y}$ & $\mathrm{N}$ & $\mathrm{N}$ \\
\hline [132] & $\mathrm{Y}$ & $\mathrm{N}$ & $\mathrm{N}$ & $\mathrm{N}$ & $\mathrm{N}$ & $\mathrm{N}$ & $\mathrm{N}$ & $\mathrm{N}$ & $\mathrm{N}$ & $\mathrm{N}$ & $\mathrm{N}$ & $\mathrm{N}$ & $\mathrm{N}$ & $\mathrm{Y}$ & $\mathrm{N}$ \\
\hline [91] & $\mathrm{Y}$ & $\mathrm{N}$ & $\mathrm{N}$ & $\mathrm{N}$ & $\mathrm{N}$ & $\mathrm{N}$ & $\mathrm{N}$ & $\mathrm{N}$ & $\mathrm{N}$ & $\mathrm{N}$ & $\mathrm{N}$ & $\mathrm{N}$ & $\mathrm{Y}$ & $\mathrm{Y}$ & Y \\
\hline [22] & $\mathrm{Y}$ & $\mathrm{N}$ & $\mathrm{N}$ & $\mathrm{N}$ & $\mathrm{N}$ & $\mathrm{N}$ & $\mathrm{N}$ & $\mathrm{N}$ & $\mathrm{N}$ & $\mathrm{Y}$ & $\mathrm{Y}$ & $\mathrm{N}$ & Y & $\mathrm{N}$ & $\mathrm{N}$ \\
\hline [133] & Y & $\mathrm{N}$ & Y & $\mathrm{Y}$ & $\mathrm{Y}$ & $\mathrm{N}$ & $\mathrm{N}$ & $\mathrm{N}$ & $\mathrm{N}$ & $\mathrm{N}$ & $\mathrm{N}$ & $Y$ & $\mathrm{~N}$ & $\mathrm{~N}$ & $\mathrm{~N}$ \\
\hline [134] & $\mathrm{N}$ & $\mathrm{N}$ & $\mathrm{N}$ & $\mathrm{N}$ & $\mathrm{N}$ & $\mathrm{N}$ & $\mathrm{N}$ & $\mathrm{N}$ & $\mathrm{N}$ & $\mathrm{Y}$ & $\mathrm{N}$ & $\mathrm{N}$ & $\mathrm{Y}$ & $\mathrm{Y}$ & $\mathrm{N}$ \\
\hline [71] & $\mathrm{Y}$ & $\mathrm{N}$ & $\mathrm{N}$ & $\mathrm{N}$ & $\mathrm{N}$ & $\mathrm{N}$ & $\mathrm{N}$ & $\mathrm{N}$ & $\mathrm{N}$ & $\mathrm{N}$ & $\mathrm{N}$ & $\mathrm{N}$ & Y & $\mathrm{Y}$ & $\mathrm{N}$ \\
\hline [45] & $\mathrm{N}$ & $\mathrm{N}$ & $\mathrm{N}$ & $\mathrm{N}$ & $\mathrm{N}$ & $\mathrm{N}$ & $\mathrm{N}$ & $\mathrm{N}$ & $\mathrm{N}$ & $\mathrm{N}$ & $\mathrm{N}$ & $Y$ & $\mathrm{~N}$ & $\mathrm{Y}$ & $\mathrm{N}$ \\
\hline [50] & $\mathrm{Y}$ & $\mathrm{N}$ & $\mathrm{N}$ & $\mathrm{N}$ & $\mathrm{N}$ & $\mathrm{N}$ & $\mathrm{N}$ & $\mathrm{N}$ & $\mathrm{N}$ & $\mathrm{Y}$ & $\mathrm{N}$ & $\mathrm{N}$ & $\mathrm{Y}$ & $\mathrm{Y}$ & $\mathrm{Y}$ \\
\hline [63] & Y & $\mathrm{Y}$ & $\mathrm{N}$ & $\mathrm{N}$ & $\mathrm{N}$ & $\mathrm{N}$ & $\mathrm{N}$ & $\mathrm{N}$ & $\mathrm{N}$ & $\mathrm{N}$ & $\mathrm{N}$ & $\mathrm{N}$ & $\mathrm{N}$ & $\mathrm{N}$ & $\mathrm{N}$ \\
\hline [136] & $\mathrm{Y}$ & $\mathrm{N}$ & $\mathrm{Y}$ & $\mathrm{N}$ & $\mathrm{N}$ & $\mathrm{N}$ & $\mathrm{N}$ & $\mathrm{N}$ & $\mathrm{Y}$ & $\mathrm{N}$ & $\mathrm{N}$ & $\mathrm{Y}$ & $\mathrm{Y}$ & $\mathrm{N}$ & $\mathrm{N}$ \\
\hline [23] & $\mathrm{Y}$ & $\mathrm{N}$ & $\mathrm{Y}$ & $\mathrm{N}$ & $\mathrm{N}$ & $\mathrm{Y}$ & $\mathrm{Y}$ & $\mathrm{N}$ & $Y$ & $\mathrm{~N}$ & $\mathrm{~N}$ & $\mathrm{~N}$ & $\mathrm{Y}$ & $\mathrm{Y}$ & Y \\
\hline [28] & $\mathrm{Y}$ & $\mathrm{N}$ & $\mathrm{N}$ & $\mathrm{N}$ & $\mathrm{N}$ & $\mathrm{N}$ & $\mathrm{N}$ & $\mathrm{N}$ & $\mathrm{N}$ & $\mathrm{N}$ & $\mathrm{N}$ & $\mathrm{N}$ & Y & $\mathrm{Y}$ & $\mathrm{Y}$ \\
\hline [137] & Y & $\mathrm{N}$ & Y & $\mathrm{N}$ & $\mathrm{N}$ & $\mathrm{N}$ & $\mathrm{N}$ & $\mathrm{N}$ & $\mathrm{N}$ & $\mathrm{N}$ & $\mathrm{Y}$ & $\mathrm{Y}$ & Y & $\mathrm{N}$ & $\mathrm{N}$ \\
\hline [138] & $\mathrm{Y}$ & $\mathrm{N}$ & $\mathrm{N}$ & $\mathrm{N}$ & $\mathrm{N}$ & $\mathrm{N}$ & $\mathrm{N}$ & $\mathrm{N}$ & $\mathrm{N}$ & $\mathrm{Y}$ & $\mathrm{Y}$ & $\mathrm{N}$ & $\mathrm{Y}$ & $\mathrm{N}$ & $\mathrm{N}$ \\
\hline [139] & $\mathrm{Y}$ & $\mathrm{N}$ & $\mathrm{N}$ & $\mathrm{N}$ & $\mathrm{N}$ & $\mathrm{N}$ & $\mathrm{N}$ & $\mathrm{N}$ & $\mathrm{N}$ & $\mathrm{N}$ & $\mathrm{N}$ & $\mathrm{N}$ & $\mathrm{N}$ & $\mathrm{Y}$ & $\mathrm{Y}$ \\
\hline [140] & $\mathrm{Y}$ & $\mathrm{N}$ & $\mathrm{N}$ & $\mathrm{N}$ & $\mathrm{N}$ & $\mathrm{N}$ & $\mathrm{N}$ & $\mathrm{N}$ & $Y$ & $\mathrm{~N}$ & $\mathrm{~N}$ & $\mathrm{~N}$ & $\mathrm{~N}$ & $\mathrm{~N}$ & $\mathrm{~N}$ \\
\hline [47] & $\mathrm{Y}$ & $\mathrm{N}$ & $\mathrm{N}$ & $\mathrm{N}$ & $\mathrm{N}$ & $\mathrm{N}$ & $\mathrm{N}$ & $\mathrm{N}$ & $\mathrm{N}$ & $\mathrm{Y}$ & $\mathrm{Y}$ & $\mathrm{N}$ & $\mathrm{N}$ & $\mathrm{Y}$ & $\mathrm{N}$ \\
\hline [141] & Y & $\mathrm{N}$ & $\mathrm{N}$ & $\mathrm{N}$ & $\mathrm{N}$ & $\mathrm{N}$ & $\mathrm{N}$ & $\mathrm{N}$ & $\mathrm{N}$ & $\mathrm{N}$ & $\mathrm{N}$ & $\mathrm{N}$ & $\mathrm{Y}$ & $\mathrm{Y}$ & $\mathrm{N}$ \\
\hline [39] & $\mathrm{Y}$ & $\mathrm{N}$ & $\mathrm{N}$ & $\mathrm{N}$ & $\mathrm{N}$ & $\mathrm{Y}$ & $\mathrm{N}$ & $\mathrm{N}$ & $\mathrm{N}$ & $\mathrm{N}$ & $\mathrm{N}$ & $\mathrm{N}$ & $\mathrm{Y}$ & $\mathrm{Y}$ & Y \\
\hline [142] & $\mathrm{Y}$ & Y & $\mathrm{Y}$ & $\mathrm{N}$ & $\mathrm{N}$ & $\mathrm{N}$ & $\mathrm{N}$ & $\mathrm{N}$ & $\mathrm{N}$ & $\mathrm{N}$ & $\mathrm{N}$ & $\mathrm{Y}$ & Y & $\mathrm{N}$ & $\mathrm{N}$ \\
\hline [143] & $Y$ & $\mathrm{~N}$ & $\mathrm{~N}$ & $\mathrm{~N}$ & $\mathrm{~N}$ & $\mathrm{~N}$ & $\mathrm{~N}$ & $\mathrm{~N}$ & $\mathrm{~N}$ & $\mathrm{Y}$ & $\mathrm{Y}$ & $\mathrm{N}$ & $\mathrm{N}$ & $\mathrm{Y}$ & $\mathrm{N}$ \\
\hline [43] & $\mathrm{Y}$ & $\mathrm{N}$ & $\mathrm{N}$ & $\mathrm{N}$ & $\mathrm{N}$ & $\mathrm{N}$ & $\mathrm{N}$ & $\mathrm{N}$ & $\mathrm{N}$ & $\mathrm{N}$ & $\mathrm{N}$ & $\mathrm{Y}$ & $\mathrm{Y}$ & $\mathrm{N}$ & $\mathrm{N}$ \\
\hline [83] & $Y$ & $\mathrm{~N}$ & $\mathrm{~N}$ & $\mathrm{~N}$ & $\mathrm{~N}$ & $\mathrm{~N}$ & $\mathrm{~N}$ & $\mathrm{~N}$ & $\mathrm{~N}$ & $Y$ & $Y$ & $\mathrm{~N}$ & $\mathrm{~N}$ & $\mathrm{~N}$ & $\mathrm{~N}$ \\
\hline
\end{tabular}


Table 9. Cont.

\begin{tabular}{|c|c|c|c|c|c|c|c|c|c|c|c|c|c|c|c|}
\hline \multirow{2}{*}{ Ref. } & \multirow{2}{*}{ VS } & \multicolumn{4}{|c|}{ Combustion } & \multicolumn{8}{|c|}{ Process Aspects } & \multicolumn{2}{|c|}{ Ash } \\
\hline & & OC & AS & REB & $\mathrm{AE}$ & 1 & 2 & 3 & 4 & 5 & 6 & 7 & Other & 8 & 9 \\
\hline [144] & $\mathrm{N}$ & $\mathrm{N}$ & $\mathrm{N}$ & $\mathrm{N}$ & $\mathrm{N}$ & $\mathrm{Y}$ & $\mathrm{Y}$ & $\mathrm{N}$ & $\mathrm{N}$ & $\mathrm{N}$ & $\mathrm{N}$ & $\mathrm{N}$ & $\mathrm{Y}$ & $\mathrm{Y}$ & $Y$ \\
\hline [145] & $\mathrm{Y}$ & $\mathrm{N}$ & $\mathrm{N}$ & $\mathrm{N}$ & $\mathrm{N}$ & $\mathrm{Y}$ & $\mathrm{Y}$ & $\mathrm{N}$ & $\mathrm{N}$ & $\mathrm{N}$ & $\mathrm{N}$ & $\mathrm{Y}$ & $\mathrm{Y}$ & $\mathrm{Y}$ & $\mathrm{Y}$ \\
\hline [90] & $\mathrm{Y}$ & $\mathrm{N}$ & $\mathrm{N}$ & $\mathrm{N}$ & $\mathrm{N}$ & $\mathrm{N}$ & $\mathrm{N}$ & $\mathrm{N}$ & $\mathrm{N}$ & $\mathrm{N}$ & $\mathrm{N}$ & $\mathrm{N}$ & $\mathrm{Y}$ & $\mathrm{Y}$ & $\mathrm{Y}$ \\
\hline [46] & $\mathrm{Y}$ & $\mathrm{N}$ & $\mathrm{N}$ & $\mathrm{N}$ & $\mathrm{N}$ & $\mathrm{N}$ & $\mathrm{N}$ & $\mathrm{N}$ & $\mathrm{N}$ & $\mathrm{Y}$ & $\mathrm{Y}$ & $\mathrm{N}$ & $\mathrm{Y}$ & $\mathrm{Y}$ & $Y$ \\
\hline [146] & $\mathrm{N}$ & $\mathrm{N}$ & $\mathrm{N}$ & $\mathrm{N}$ & $\mathrm{N}$ & $\mathrm{Y}$ & $\mathrm{Y}$ & $\mathrm{N}$ & $\mathrm{N}$ & $\mathrm{N}$ & $\mathrm{N}$ & $\mathrm{Y}$ & $\mathrm{Y}$ & $\mathrm{N}$ & $\mathrm{N}$ \\
\hline [147] & $\mathrm{Y}$ & $\mathrm{N}$ & $\mathrm{N}$ & $\mathrm{N}$ & $\mathrm{N}$ & $\mathrm{Y}$ & $\mathrm{Y}$ & $\mathrm{N}$ & $\mathrm{N}$ & $\mathrm{N}$ & $\mathrm{N}$ & $\mathrm{N}$ & $\mathrm{Y}$ & $\mathrm{Y}$ & $Y$ \\
\hline [148] & $\mathrm{N}$ & $\mathrm{N}$ & $\mathrm{N}$ & $\mathrm{N}$ & $\mathrm{N}$ & $\mathrm{N}$ & $\mathrm{N}$ & $\mathrm{N}$ & $\mathrm{N}$ & $\mathrm{Y}$ & $\mathrm{Y}$ & $\mathrm{N}$ & $\mathrm{Y}$ & $\mathrm{N}$ & $\mathrm{N}$ \\
\hline [88] & $\mathrm{Y}$ & $\mathrm{N}$ & $\mathrm{N}$ & $\mathrm{N}$ & $\mathrm{Y}$ & $\mathrm{Y}$ & $\mathrm{Y}$ & $\mathrm{N}$ & $\mathrm{N}$ & $\mathrm{N}$ & $\mathrm{N}$ & $\mathrm{N}$ & $\mathrm{Y}$ & $\mathrm{Y}$ & $Y$ \\
\hline [24] & $\mathrm{Y}$ & $\mathrm{N}$ & $\mathrm{N}$ & $\mathrm{N}$ & $\mathrm{N}$ & $\mathrm{N}$ & $\mathrm{N}$ & $\mathrm{N}$ & $\mathrm{Y}$ & $\mathrm{Y}$ & $Y$ & $\mathrm{~N}$ & $\mathrm{Y}$ & $\mathrm{N}$ & $\mathrm{N}$ \\
\hline [149] & $\mathrm{Y}$ & $\mathrm{N}$ & $\mathrm{Y}$ & $\mathrm{N}$ & $\mathrm{N}$ & $\mathrm{N}$ & $\mathrm{N}$ & $\mathrm{N}$ & $\mathrm{Y}$ & $\mathrm{N}$ & $\mathrm{N}$ & $\mathrm{N}$ & $\mathrm{Y}$ & $\mathrm{N}$ & $\mathrm{N}$ \\
\hline [150] & $\mathrm{Y}$ & $\mathrm{N}$ & $\mathrm{N}$ & $\mathrm{N}$ & $\mathrm{N}$ & $\mathrm{N}$ & $\mathrm{N}$ & $\mathrm{N}$ & $\mathrm{N}$ & $\mathrm{Y}$ & $\mathrm{Y}$ & $\mathrm{N}$ & $\mathrm{N}$ & $\mathrm{Y}$ & $Y$ \\
\hline [29] & $\mathrm{N}$ & $\mathrm{N}$ & $\mathrm{Y}$ & $\mathrm{N}$ & $\mathrm{N}$ & $\mathrm{N}$ & $\mathrm{N}$ & $\mathrm{N}$ & $\mathrm{N}$ & $\mathrm{N}$ & $\mathrm{N}$ & $\mathrm{N}$ & $\mathrm{Y}$ & $\mathrm{Y}$ & $\mathrm{N}$ \\
\hline [59] & $\mathrm{N}$ & $\mathrm{N}$ & $\mathrm{Y}$ & $\mathrm{N}$ & $\mathrm{N}$ & $\mathrm{N}$ & $\mathrm{N}$ & $\mathrm{N}$ & $\mathrm{Y}$ & $\mathrm{N}$ & $\mathrm{N}$ & $\mathrm{Y}$ & Y & $\mathrm{N}$ & $\mathrm{N}$ \\
\hline [151] & $\mathrm{Y}$ & $\mathrm{N}$ & $\mathrm{Y}$ & $\mathrm{Y}$ & $\mathrm{Y}$ & $\mathrm{N}$ & $\mathrm{N}$ & $\mathrm{N}$ & $\mathrm{N}$ & $\mathrm{N}$ & $\mathrm{N}$ & $\mathrm{Y}$ & $\mathrm{Y}$ & $\mathrm{N}$ & $\mathrm{N}$ \\
\hline [152] & $\mathrm{Y}$ & $\mathrm{N}$ & $\mathrm{N}$ & $\mathrm{N}$ & $\mathrm{N}$ & $\mathrm{N}$ & $\mathrm{N}$ & $\mathrm{N}$ & $\mathrm{N}$ & $\mathrm{N}$ & $\mathrm{N}$ & $\mathrm{N}$ & $\mathrm{Y}$ & $\mathrm{N}$ & $\mathrm{N}$ \\
\hline [21] & $\mathrm{Y}$ & $\mathrm{N}$ & $\mathrm{N}$ & $\mathrm{N}$ & $\mathrm{N}$ & $\mathrm{Y}$ & $\mathrm{Y}$ & $\mathrm{N}$ & $\mathrm{Y}$ & $\mathrm{N}$ & $\mathrm{N}$ & $\mathrm{Y}$ & $\mathrm{Y}$ & $\mathrm{Y}$ & $\mathrm{N}$ \\
\hline [153] & $\mathrm{Y}$ & $\mathrm{Y}$ & $\mathrm{Y}$ & $\mathrm{N}$ & $\mathrm{N}$ & $\mathrm{N}$ & $\mathrm{N}$ & $\mathrm{N}$ & $\mathrm{N}$ & $\mathrm{N}$ & $\mathrm{N}$ & $\mathrm{Y}$ & $\mathrm{Y}$ & $\mathrm{N}$ & $\mathrm{N}$ \\
\hline [40] & $\mathrm{Y}$ & $\mathrm{N}$ & $\mathrm{N}$ & $\mathrm{N}$ & $\mathrm{N}$ & $\mathrm{Y}$ & $\mathrm{Y}$ & $\mathrm{N}$ & $\mathrm{N}$ & $\mathrm{N}$ & $\mathrm{N}$ & $\mathrm{N}$ & $\mathrm{Y}$ & $\mathrm{Y}$ & $\mathrm{Y}$ \\
\hline [154] & Y & $\mathrm{N}$ & $\mathrm{N}$ & $\mathrm{N}$ & $\mathrm{N}$ & $\mathrm{N}$ & $\mathrm{N}$ & $\mathrm{N}$ & $\mathrm{N}$ & $\mathrm{Y}$ & $\mathrm{Y}$ & $\mathrm{N}$ & $\mathrm{N}$ & $\mathrm{N}$ & $\mathrm{N}$ \\
\hline [27] & $\mathrm{Y}$ & $\mathrm{N}$ & $\mathrm{N}$ & $\mathrm{N}$ & $\mathrm{N}$ & $\mathrm{N}$ & $\mathrm{N}$ & $\mathrm{N}$ & $\mathrm{N}$ & $\mathrm{Y}$ & $\mathrm{Y}$ & $\mathrm{N}$ & $\mathrm{N}$ & $\mathrm{N}$ & $\mathrm{N}$ \\
\hline [48] & $\mathrm{Y}$ & $\mathrm{N}$ & $\mathrm{N}$ & $\mathrm{N}$ & $\mathrm{N}$ & $\mathrm{N}$ & $\mathrm{N}$ & $\mathrm{N}$ & $\mathrm{N}$ & $\mathrm{Y}$ & $\mathrm{N}$ & $\mathrm{N}$ & $\mathrm{Y}$ & $\mathrm{N}$ & $\mathrm{N}$ \\
\hline [156] & $\mathrm{Y}$ & $\mathrm{N}$ & $\mathrm{N}$ & $\mathrm{N}$ & $\mathrm{N}$ & $\mathrm{N}$ & $\mathrm{N}$ & $\mathrm{N}$ & $\mathrm{N}$ & $\mathrm{Y}$ & $\mathrm{N}$ & $\mathrm{N}$ & $\mathrm{Y}$ & $\mathrm{N}$ & $\mathrm{N}$ \\
\hline [157] & $\mathrm{Y}$ & $\mathrm{N}$ & $\mathrm{N}$ & $\mathrm{N}$ & $\mathrm{N}$ & $\mathrm{N}$ & $\mathrm{N}$ & $\mathrm{N}$ & $\mathrm{Y}$ & $\mathrm{Y}$ & $\mathrm{N}$ & $\mathrm{N}$ & $\mathrm{N}$ & $\mathrm{N}$ & $\mathrm{N}$ \\
\hline [158] & $Y$ & $\mathrm{~N}$ & $\mathrm{~N}$ & $\mathrm{~N}$ & $\mathrm{~N}$ & $\mathrm{~N}$ & $\mathrm{~N}$ & $\mathrm{~N}$ & $\mathrm{~N}$ & $\mathrm{Y}$ & $\mathrm{N}$ & $\mathrm{N}$ & $\mathrm{Y}$ & $\mathrm{Y}$ & $Y$ \\
\hline
\end{tabular}

Pedersen et al. (1996) [18] reported on full scale straw-coal co-combustion in a pulverized coal boiler. No discernible trend in the carbon content in ash was observed for the 10 and $20 \%$ biomass share trials, respectively, compared to coal combustion only. A similar conclusion was reached by Savolainen (2003) [106] on evaluating pulverized coal and sawdust co-combustion in a large power plant boiler with up to $25 \% \mathrm{wt}$. of biomass share in blended fuel. In addition to comparable results obtained, Skodras et al. (2002) [44] concluded that more unburned carbon was found in fly ash (up to 6\% wt.) than in bottom ash (typically around $2 \%$ wt.). Steer et al. (2013) [120] analyzed full scale miscanthus co-combustion in coal power plant boilers, concluding that carbon burnout was seriously affected by biomass particle size fed to the boilers and, to some extent, also by secondary air amount and distribution. Increased slagging tendency was observed with the increasing miscanthus share in the combusted fuel. Kalembkiewicz and Chmielarz (2012) [51] reviewed several papers dealing with carbon burnout with various fuel blends combusted, finding examples of both increased and decreased carbon content in ash reported in co-combustion tests and identified particle size, fuel moisture, and burner type as the most important factors determining the final carbon content in ash.

Fly ash from co-combustion can be utilized in concrete production as proposed by Baxter (2005) [35] and Leckner (2007) [36] due to its confirmed high pozzolanic activity [51]. Co-combustion fly ash from full scale applications in Netherlands utilizing various biomass and waste types were examined by Sarabèr (2014) [179] as possible mortar or concrete additives, confirming their general suitability for these purposes despite their variable chemical composition and the earlier evidence summed up by Sondreal et al. (2001) [15].

Table 9 indicates the research scope of relevant literal sources in operational and process aspects. 


\section{Combustion Process Modifications: Air Staging, Oxycombustion, Flue Gas Recirculation, and Reburning}

Along with the sole biomass co-combustion fraction effect on combustion characteristics and combustor operational issues, several other process modifications were tested in parallel [119,129], including both measures commonly applied in fossil fuel power plant boilers to decrease $\mathrm{NO}_{\mathrm{x}}$ emissions (air staging, flue gas recirculation, fuel reburning) $[133,151,180]$ as well as oxycombustion, which should improve boiler thermal efficiency and allow for effective carbon capture from flue gas [42,123]. These can be successfully applied both in heat and power production as well as in heavy industry (steel, clinker, or aluminum production).

Oxycombustion can be applied in most industrial boilers and furnaces [181] but its potential for fuel saving is especially high in the aluminum production industry, where it has been estimated to be as high as $50 \%[182,183]$. In addition, it can contribute to charge melting time shortening, as presented by Dzurňák et al. (2019) [184] and thus improve the melting process productivity and its environmental impact [185]. In combination with other measures including improved heat recovery [186] and the use of biofuels and power from biomass [187], substantial reduction in GHG emissions from aluminum production can be achieved.

The performed studies concluded that $\mathrm{NO}_{\mathrm{x}}$ emission reduction by air staging, flue gas recirculation, and fuel reburning can further be enhanced by biomass co-combustion, as the volatiles released in early stages of biomass combustion serve as an effective agent for nitrous oxides reduction. However, certain problems with carbon burnout were reported, and a compromise between $\mathrm{NO}_{\mathrm{x}}$ emissions reduction and thermal efficiency loss has to be sought. Oxycombustion can alleviate this negative effect, and it is often applied in combination with some of the above measures $[123,142,153]$. Table 9 provides an overview of these measures applied either individually or in parallel.

\section{Emissions of Greenhouse Gases and Other Pollutants}

Carbon dioxide emissions in the co-combustion mode are described in Section 2. Here, other gaseous emissions, namely $\mathrm{NO}_{\mathrm{x}}, \mathrm{SO}_{\mathrm{x}}, \mathrm{CO}$, and others, are considered.

Tchapda and Pisupati (2014) [11] analyzed the co-combustion technology in a voluminous review paper, identifying both fuel and air nitrogen as significant contributors to nitrogen oxides emissions, regardless of whether fossil fuel or biomass is combusted. Thus, lower nitrogen content in biomass compared to coal generally led to a decrease in $\mathrm{NO}_{\mathrm{x}}$ emissions [49]; however, this issue is more complex, and cases with increased $\mathrm{NO}_{\mathrm{x}}$ emissions during co-combustion have also been reported [35]. This is corroborated by the results of experimental combustor of woody biomass-coal co-combustion trials performed by Yelverton et al. (2020) [155] that yielded higher, equal, or lower $\mathrm{NO}_{\mathrm{x}}$ emissions in 20 as well as in $40 \%$ biomass mass share trials with no discernible trend. Both Sondreal et al. (2001) and Baxter (2005) $[15,35]$ argued that biomass often contains more moisture than coal, which leads to combustion temperature decrease and reduced $\mathrm{NO}_{\mathrm{x}}$ emissions. This was confirmed in industrial scale experiments described by Wieck-Hanssen et al. (2000) [105], when coal and straw co-combustion yielded almost the same $\mathrm{NO}_{\mathrm{x}}$ emissions as the combustion of coal only. As pointed out by Karampinis et al. (2014) [10], attention has to be paid to suitable air distribution to allow for efficient combustion of volatiles released from biomass as well as to air staging further reducing the $\mathrm{NO}_{\mathrm{x}}$ emissions. Other means of nitrogen oxides reduction comprise reburning and flue gas recirculation as discussed in the previous chapter.

Co-combustion has been proven to reduce sulfur oxides emissions due to effective sulfur recapture by calcium if present in the co-combusted biomass or waste ash in higher content than in fossil fuel ash and especially in fluid boilers, enabling far better contact of flue gas with ash than grate boilers [10,31]. This biomass effect can be combined with limestone addition to decrease the sulfur oxides emissions even further, as documented by Atimtay et al. (2017) [85]. Increasing combustion temperature reduces the sulfur capture 
efficiency. Chen et al. (2018) [134] conducted thermogravimetric measurements of sewage sludge, coal, shiitake substrate, and their blends combustion to assess the synergistic effect of biomass addition on the energetic and environmental combustion indicators. Sludgecoal co-combustion proved the most effective in $\mathrm{CH}_{4}, \mathrm{NO}_{\mathrm{x}}$, and $\mathrm{SO}_{2}$ emissions reduction, whereas sludge-shiitake co-combustion led to an increase in $\mathrm{CH}_{4}$ and $\mathrm{NO}_{\mathrm{x}}$ emissions at the sludge share of $25 \%$ with their gradual decrease on further sludge share increase. Skodras et al. (2002) [44] confirmed the sulfur oxides emissions reduction in wood-lignite co-combustion experiments in an industrial boiler, and similar conclusions were drawn by Steer et al. (2013) [120] after miscanthus-coal co-combustion tests in a $500 \mathrm{MW}_{\mathrm{t}}$ power plant boiler.

No clear trend can generally be drawn in $\mathrm{CO}$ emissions, being often more dependent on air excess and distribution than on the co-combustion ratio, as stated by Skodras et al. (2002) and Lukáč et al. (2019) [44,188]. Sami et al. (2001) [8], on the other hand, summed up the results of several industrial co-combustion trials and concluded lower $\mathrm{CO}$ emissions of most of them than in sole fossil fuel combustion, along with $\mathrm{SO}_{\mathrm{x}}$ and $\mathrm{NO}_{\mathrm{x}}$ emission decrease. Biomass pre-drying has a certain positive role in $\mathrm{CO}$ emissions reduction, as argued by Verma et al. (2017) [52]. Steer et al. (2013) [120] observed an increase in CO emission in the miscanthus-coal co-combustion compared to coal combustion only, which they attributed to insufficient air delivery for complete combustion of volatiles rapidly released from miscanthus. The same effect was confirmed for $\mathrm{CO}$ emissions increase in bagasse-coal co-combustion studied by Bragato et al. (2012) [113]. Recent woody biomasscoal co-combustion trials performed by Yelverton et al. (2020) [155] did not provide any clear dependence of $\mathrm{CO}$ emission on the biomass share or type.

Table 10 provides a structured view on the type of emissions investigated in multiple studies. While almost all papers analyzed the $\mathrm{NO}_{\mathrm{x}}$ and $\mathrm{SO}_{\mathrm{x}}$ emissions, fewer of them dealt with $\mathrm{CO}$ and yet fewer investigated other gaseous emissions such as VOC (volatile organic compounds), dioxins, metals, or chlorine emissions.

Table 10. Overview of papers on emissions of greenhouse gases and other compounds. Legend: Eq = recalculated to $\mathrm{CO}_{2}$ equivalent as a part of $\mathrm{CO}_{2} \mathrm{LCA}, \mathrm{LCA}=$ life cycle assessment, $\mathrm{GHG}=$ greenhouse gases $(\mathrm{Y}=$ investigated, $\mathrm{N}=$ not investigated), GWP = global warming potential.

\begin{tabular}{|c|c|c|c|c|c|c|c|}
\hline \multirow{2}{*}{ Ref. } & \multicolumn{4}{|c|}{ GHG Emissions } & \multicolumn{3}{|c|}{ Other Emissions } \\
\hline & $\mathrm{NO}_{\mathbf{x}}$ & $\mathrm{SO}_{\mathrm{x}}$ & $\mathrm{CO}$ & Other & Metals & Chlorides & Other \\
\hline [18] & Y & $\mathrm{Y}$ & $\mathrm{N}$ & $\mathrm{N}$ & $\mathrm{N}$ & $\mathrm{N}$ & $\mathrm{N}$ \\
\hline [17] & $\mathrm{Y}$ & $\mathrm{Y}$ & $\mathrm{Y}$ & $\mathrm{Y}$ & $\mathrm{Y}$ & $\mathrm{Y}$ & $\mathrm{Y}$ \\
\hline [104] & $\mathrm{Y}$ & $\mathrm{N}$ & $\mathrm{N}$ & $\mathrm{N}$ & $\mathrm{N}$ & $\mathrm{N}$ & $\mathrm{N}$ \\
\hline [19] & $\mathrm{Y}$ & $\mathrm{N}$ & $\mathrm{N}$ & $\mathrm{N}$ & $\mathrm{N}$ & $\mathrm{N}$ & $\mathrm{N}$ \\
\hline [105] & $\mathrm{Y}$ & $\mathrm{Y}$ & $\mathrm{N}$ & $\mathrm{Y}$ & $\mathrm{Y}$ & $\mathrm{Y}$ & $\mathrm{Y}$ \\
\hline [64] & Y & $\mathrm{Y}$ & $\mathrm{N}$ & $\mathrm{Y}$ & $\mathrm{Y}$ & $\mathrm{N}$ & $\mathrm{N}$ \\
\hline [8] & $\mathrm{Y}$ & $\mathrm{Y}$ & $\mathrm{Y}$ & $\mathrm{N}$ & $\mathrm{N}$ & $\mathrm{Y}$ & $\mathrm{N}$ \\
\hline [65] & Y eq & Y eq & Y eq & Y eq & $\mathrm{Y}$ & $\mathrm{N}$ & $\mathrm{Y}$ \\
\hline [44] & $Y^{1}$ & $Y^{I}$ & $Y^{1}$ & $Y^{1}$ & $\mathrm{Y}$ & $\mathrm{N}$ & Y \\
\hline [82] & Y & $\mathrm{N}$ & $\mathrm{Y}$ & $\mathrm{N}$ & $\mathrm{N}$ & $\mathrm{N}$ & $\mathrm{N}$ \\
\hline [106] & Y & $\mathrm{Y}$ & $\mathrm{N}$ & $\mathrm{N}$ & $\mathrm{N}$ & $\mathrm{N}$ & $\mathrm{Y}$ \\
\hline [89] & Y & $\mathrm{N}$ & $\mathrm{N}$ & $\mathrm{N}$ & $\mathrm{N}$ & $\mathrm{N}$ & $\mathrm{N}$ \\
\hline [35] & $\mathrm{Y}$ & $\mathrm{Y}$ & $\mathrm{N}$ & $\mathrm{N}$ & $\mathrm{N}$ & $\mathrm{N}$ & $\mathrm{N}$ \\
\hline [107] & $\mathrm{Y}$ & $\mathrm{N}$ & $\mathrm{N}$ & $\mathrm{N}$ & $\mathrm{N}$ & $\mathrm{N}$ & $\mathrm{N}$ \\
\hline [36] & $\mathrm{Y}$ & $\mathrm{Y}$ & $\mathrm{Y}$ & $\mathrm{Y}$ & Y & $\mathrm{Y}$ & $\mathrm{Y}$ \\
\hline [33] & $\mathrm{Y}$ & $\mathrm{Y}$ & $\mathrm{N}$ & $\mathrm{N}$ & $\mathrm{N}$ & $\mathrm{N}$ & $\mathrm{N}$ \\
\hline [13] & $\mathrm{Y}$ & $\mathrm{Y}$ & $\mathrm{Y}$ & $\mathrm{Y}$ & Y & Y & Y \\
\hline [32] & Y & $\mathrm{Y}$ & $\mathrm{N}$ & Y & $\mathrm{Y}$ & $\mathrm{N}$ & $\mathrm{N}$ \\
\hline [110] & $\mathrm{Y}$ & $\mathrm{N}$ & $\mathrm{N}$ & $\mathrm{N}$ & $\mathrm{N}$ & $\mathrm{N}$ & $\mathrm{N}$ \\
\hline [111] & $\mathrm{Y}$ & $\mathrm{N}$ & $\mathrm{Y}$ & $\mathrm{N}$ & $\mathrm{N}$ & $\mathrm{N}$ & $\mathrm{N}$ \\
\hline [51] & $\mathrm{N}$ & $\mathrm{N}$ & $\mathrm{N}$ & $\mathrm{N}$ & $\mathrm{Y}$ & $\mathrm{Y}$ & $\mathrm{Y}$ \\
\hline [112] & $\mathrm{Y}$ & $\mathrm{N}$ & $\mathrm{N}$ & $\mathrm{N}$ & $\mathrm{N}$ & $\mathrm{N}$ & $\mathrm{N}$ \\
\hline
\end{tabular}


Table 10. Cont.

\begin{tabular}{|c|c|c|c|c|c|c|c|}
\hline \multirow{2}{*}{ Ref. } & \multicolumn{4}{|c|}{ GHG Emissions } & \multicolumn{3}{|c|}{ Other Emissions } \\
\hline & $\mathrm{NO}_{\mathrm{x}}$ & $\mathrm{SO}_{\mathrm{x}}$ & $\mathrm{CO}$ & Other & Metals & Chlorides & Other \\
\hline [113] & $\mathrm{N}$ & $\mathrm{N}$ & Y & Y & $\mathrm{N}$ & $\mathrm{N}$ & $\mathrm{N}$ \\
\hline [114] & Y eq & Y eq & Y eq & Y eq & $\mathrm{N}$ & $\mathrm{N}$ & $\mathrm{N}$ \\
\hline [68] & Y & $Y^{1}$ & $Y$ & $Y^{1}$ & $\mathrm{~N}$ & $\mathrm{~N}$ & $\mathrm{~N}$ \\
\hline [119] & Y & $\mathrm{N}$ & Y & $\mathrm{N}$ & $\mathrm{N}$ & $\mathrm{N}$ & $\mathrm{N}$ \\
\hline [120] & Y & Y & Y & Y & $\mathrm{N}$ & $\mathrm{N}$ & $\mathrm{N}$ \\
\hline [31] & Y & Y & $\mathrm{N}$ & $\mathrm{N}$ & $\mathrm{N}$ & $\mathrm{N}$ & $\mathrm{N}$ \\
\hline [121] & Y & $\mathrm{N}$ & Y & $\mathrm{N}$ & $\mathrm{N}$ & $\mathrm{N}$ & $\mathrm{N}$ \\
\hline [11] & Y & Y & $\mathrm{N}$ & $\mathrm{N}$ & $\mathrm{N}$ & $\mathrm{N}$ & $\mathrm{N}$ \\
\hline [10] & Y & Y & Y & Y & $\mathrm{N}$ & $\mathrm{N}$ & $\mathrm{N}$ \\
\hline$[86]$ & Y & Y & $\mathrm{N}$ & $\mathrm{N}$ & $\mathrm{N}$ & $\mathrm{N}$ & $\mathrm{N}$ \\
\hline [41] & Y & $\mathrm{N}$ & $\mathrm{N}$ & $\mathrm{N}$ & $\mathrm{N}$ & $\mathrm{N}$ & $\mathrm{N}$ \\
\hline [125] & Y & $\mathrm{N}$ & Y & $\mathrm{N}$ & $\mathrm{N}$ & $\mathrm{N}$ & $\mathrm{N}$ \\
\hline [49] & Y & $\mathrm{N}$ & $\mathrm{N}$ & $\mathrm{N}$ & $\mathrm{N}$ & $\mathrm{N}$ & $\mathrm{N}$ \\
\hline [69] & Y & Y & $\mathrm{N}$ & $\mathrm{N}$ & $\mathrm{N}$ & $\mathrm{N}$ & $\mathrm{N}$ \\
\hline [126] & Y & $\mathrm{N}$ & Y & $\mathrm{N}$ & $\mathrm{N}$ & $\mathrm{N}$ & $\mathrm{N}$ \\
\hline [58] & $\mathrm{N}$ & Y & Y & $\mathrm{N}$ & $\mathrm{N}$ & $\mathrm{N}$ & $\mathrm{N}$ \\
\hline [127] & Y & Y & Y & Y & $\mathrm{N}$ & $\mathrm{N}$ & $\mathrm{N}$ \\
\hline [85] & Y & Y & Y & Y & $\mathrm{N}$ & $\mathrm{N}$ & $\mathrm{N}$ \\
\hline [84] & Y & Y & $\mathrm{N}$ & $\mathrm{N}$ & $\mathrm{N}$ & $\mathrm{N}$ & $\mathrm{N}$ \\
\hline [56] & \multicolumn{7}{|c|}{ Part of $\mathrm{CO}_{2} \mathrm{LCA}$, recalculated to $\mathrm{CO}_{2}$ equivalent } \\
\hline [52] & $\mathrm{Y}$ & Y & $\mathrm{Y}$ & Y & $\mathrm{N}$ & $\mathrm{N}$ & $\mathrm{N}$ \\
\hline [42] & $\mathrm{Y}$ & Y & $\mathrm{Y}$ & Y & $\mathrm{N}$ & $\mathrm{N}$ & $\mathrm{N}$ \\
\hline [20] & $\mathrm{Y}$ & Y & $\mathrm{Y}$ & $\mathrm{N}$ & $\mathrm{N}$ & $\mathrm{N}$ & $\mathrm{N}$ \\
\hline [132] & $\mathrm{Y}$ & $\mathrm{Y}$ & $\mathrm{Y}$ & Y & $\mathrm{N}$ & $\mathrm{N}$ & $\mathrm{N}$ \\
\hline [91] & $\mathrm{Y}$ & $\mathrm{Y}$ & $\mathrm{N}$ & $\mathrm{N}$ & $\mathrm{N}$ & $\mathrm{N}$ & $\mathrm{N}$ \\
\hline [22] & $\mathrm{Y}$ & Y & $\mathrm{Y}$ & $\mathrm{N}$ & $\mathrm{N}$ & $\mathrm{N}$ & $\mathrm{N}$ \\
\hline [133] & $\mathrm{Y}$ & $\mathrm{N}$ & $\mathrm{N}$ & $\mathrm{N}$ & $\mathrm{N}$ & $\mathrm{N}$ & $\mathrm{N}$ \\
\hline [134] & $\mathrm{Y}$ & $\mathrm{Y}$ & Y & $\mathrm{N}$ & $\mathrm{N}$ & $\mathrm{N}$ & $\mathrm{N}$ \\
\hline [71] & Y & Y & Y & $\mathrm{N}$ & $\mathrm{N}$ & $\mathrm{N}$ & $\mathrm{N}$ \\
\hline$[45]$ & $\mathrm{N}$ & $\mathrm{N}$ & $\mathrm{N}$ & $\mathrm{N}$ & Y & $\mathrm{N}$ & Y \\
\hline [135] & Y & Y & Y & Y & $\mathrm{N}$ & $\mathrm{N}$ & Y \\
\hline [136] & Y & $\mathrm{N}$ & Y & $\mathrm{N}$ & $\mathrm{N}$ & $\mathrm{N}$ & $\mathrm{N}$ \\
\hline [23] & Y & Y & Y & $\mathrm{N}$ & $\mathrm{N}$ & $\mathrm{N}$ & $\mathrm{N}$ \\
\hline [137] & $\mathrm{N}$ & $\mathrm{N}$ & Y & Y & $\mathrm{N}$ & $\mathrm{N}$ & $\mathrm{N}$ \\
\hline [138] & $\mathrm{N}$ & $\mathrm{N}$ & $\mathrm{N}$ & Y & Y & $\mathrm{N}$ & $\mathrm{N}$ \\
\hline [70] & \multicolumn{7}{|c|}{ Part of $\mathrm{CO}_{2} \mathrm{LCA}$ recalculated to $\mathrm{CO}_{2} \mathrm{GWP}$ equivalent } \\
\hline [140] & Y & Y & $\mathrm{N}$ & $\mathrm{N}$ & $N$ & $\mathrm{~N}$ & $\mathrm{~N}$ \\
\hline [142] & Y & Y & Y & Y & $\mathrm{N}$ & $\mathrm{N}$ & $\mathrm{N}$ \\
\hline [43] & Y & Y & Y & $\mathrm{N}$ & $\mathrm{N}$ & $\mathrm{N}$ & $\mathrm{N}$ \\
\hline [46] & $\mathrm{N}$ & $\mathrm{N}$ & $\mathrm{N}$ & $\mathrm{N}$ & Y & $\mathrm{N}$ & $\mathrm{N}$ \\
\hline [146] & Y & Y & $\mathrm{N}$ & Y & $\mathrm{N}$ & $\mathrm{N}$ & $\mathrm{N}$ \\
\hline [149] & $\mathrm{N}$ & Y & Y & $\mathrm{N}$ & $\mathrm{N}$ & $\mathrm{N}$ & $\mathrm{N}$ \\
\hline [29] & Y & Y & Y & $\mathrm{N}$ & Y & $\mathrm{N}$ & $\mathrm{N}$ \\
\hline [59] & Y & $\mathrm{N}$ & $\mathrm{N}$ & $\mathrm{N}$ & $\mathrm{N}$ & $\mathrm{N}$ & $\mathrm{N}$ \\
\hline [151] & Y & $\mathrm{N}$ & $\mathrm{N}$ & Y & $\mathrm{N}$ & $\mathrm{N}$ & $\mathrm{N}$ \\
\hline [152] & $\mathrm{N}$ & $\mathrm{N}$ & Y & Y & $\mathrm{N}$ & $\mathrm{N}$ & $\mathrm{N}$ \\
\hline [153] & Y & $\mathrm{N}$ & Y & $\mathrm{N}$ & $\mathrm{N}$ & $\mathrm{N}$ & $\mathrm{N}$ \\
\hline [155] & Y & Y & Y & Y & $\mathrm{N}$ & $\mathrm{N}$ & Y \\
\hline [48] & Y & Y & Y & $\mathrm{N}$ & $\mathrm{N}$ & $\mathrm{N}$ & Y \\
\hline [156] & $\mathrm{N}$ & Y & Y & $\mathrm{N}$ & $\mathrm{N}$ & $\mathrm{N}$ & $\mathrm{N}$ \\
\hline [157] & Y & Y & $\mathrm{N}$ & $\mathrm{N}$ & $\mathrm{N}$ & $\mathrm{N}$ & $\mathrm{N}$ \\
\hline
\end{tabular}

Finney et al. (2018) [45] investigated metal aerosol emissions during biomass and coal combustion in a pilot scale combustor. Coal contained more than $3 \%$ wt. of ash on dry basis, whereas that in the woody biomass was below $0.8 \% \mathrm{wt}$. Potassium emissions 
from woody biomass combustion were more than six times higher, but emissions of other alkali and alkali earth metals were significantly lower than those from coal combustion. Nickel, zinc, and chromium emissions were comparable in both cases following no clear dependency on their content in ash. Emissions of other metals were significantly lower in the case of biomass combustion, which confirmed the earlier findings of Hein and Bemtgen (1998) and Dai et al. (2008) [13,17]. Guo and Zhong (2018) [138] performed combustion experiments with coal and composite biomass pellets in a fluidized bed combustor. They observed a synergic effect of both fuels co-combustion, leading to lower polyaromatic hydrocarbons emissions than expected in a broad range of combustion temperatures. The same result was obtained for trace metals emissions expressed in terms of volatilization rate reduction, which the authors attributed to chemical interactions of alkalis in biomass ash capturing other metals. Their findings related to polyaromatic hydrocarbons emissions corroborated the earlier conclusions of Bragato et al. (2012) [113] obtained in coal-bagasse co-combustion tests in a wide combustion temperatures interval. Skodras et al. (2002) [44] performed a study on coal and various woody fuels co-combustion in an industrial boiler dedicated to dioxins and metals emissions assessment. Dioxins emissions could neither be linked to carbon burnout in ash nor to co-combusted biomass type. Metals' emissions were lower than expected based on pure coal or biomass combustion trials. More recent studies regarding the gaseous emissions from co-combustion processes were published by Fu et al. (2019) [46] and Xue et al. (2020) [29]. Xue et al. (2020) [29] found that co-combustion of coal and wheat straw in a fluidized bed combustor restrained zinc, cadmium, and lead from release in flue gas, while the release of chromium was promoted. They also observed a significant impact of secondary air introduction on the metals' emissions, indicating no effect on chromium emissions but an increased effect of zinc, cadmium, and lead. An increase of volatilization ratio for copper, chromium, and zinc was disclosed by Fu et al. (2019) [46] in coal-sewage sludge co-combustion experiments in a thermogravimetric analyzer, while that of arsenic decreased and that of nickel and lead remained unchanged. It can thus be concluded that while there is enough evidence of the decrease of polyaromatic and carcinogenic emissions release during co-combustion, the release of metals is a complex problem and needs to be studied further, both in co-combustion studies and in separate combustion of fossil and renewable fuels in industrial [189] as well as domestic boilers [190].

\section{Challenges and Future Development}

The review of relevant literature highlighted the rapid technologic advances in biomass and waste co-combustion with fossil fuels. However, several weak points need to be further addressed and should be a part of future co-combustion technology and equipment development:

- Deeper understanding of carbon and GHG emissions lifecycles in co-combustion applications is needed. While using local sources of waste biomass and other wastes has the potential to reduce the GHG emissions significantly, on-purpose growing, harvesting, transporting, and co-combusting biomass should be studied carefully. The resulting net GHG balance can be close to zero or even negative in some cases, as demonstrated by Miedema et al. (2017) [56].

- There is no single recipe or standardized approach to specify optimal co-combustion share, plant size, and layout. Regional and local aspects must be always analyzed regarding the resources availability and variability, plant size and layout, biomass and waste pretreatment possibilities, the resulting GHG, and other emissions and ash utilization, as well as long term effect of fouling and possible corrosion-all this shapes the optimal design and operation of each particular co-combustion installation.

- Biomass processing routes to fuels and chemicals play an important role in decision making on optimal biomass and waste utilization, as they advance towards their full commercialization, possibly restraining co-combustion application to low quality or contaminated or otherwise unfavorable fuels. The development of electro mobility on 
the other hand might enhance the co-combustion application in low carbon power production as a result of increased electricity consumption.

- Advances in gasification and pyrolysis processes of mixed fossil and biomass/waste fuels are necessary to make those technologies fully available and competitive. Further process modifications, including new methods for biomass pretreatment, should be developed regarding $\mathrm{NO}_{\mathrm{x}}$ and other GHG emissions decrease and reduced risk of equipment corrosion.

\section{Conclusions}

Co-combustion represents a viable and widely applied technology with the potential to reduce GHG emissions from heat and power generation in near and mid-term future, especially with local biomass and waste sources exploitation. Retrofit of existing fossil fuel boilers is associated with low specific cost and reasonable co-combustion levels of up to $20 \%$ wt. Most analyzed studies concluded that no significant tendencies in fouling and equipment corrosion were identified unless for co-combustion of combined high-ash and high-sulfur content biomass (herbaceous biomass).

Rational application of air staging, flue gas recirculation, and fuel reburning in cocombustion applications can further enhance their positive synergic effect towards significant reduction of nitrogen oxides emissions without significant increase of carbon monoxide emissions and unburned carbon in ash. If combined with oxycombustion, an additional bonus in increased combustor thermal efficiency can be obtained. Feed type and moisture content, combustor type, and air distribution were identified as relevant factors affecting the GHG emissions, which pose a challenge for continuous development of cost-effective feed pretreatment methods and combustor design optimization. As proven by numerous studies, biomass ash is capable of sulfur capture, thus reducing sulfur oxides emissions. Emission trends of polyaromatics and other carcinogenic substances in the co-combustion regime deserve further research. The well documented volatilization and emissions of alkali and alkali earth metals during co-combustion is closely related to slagging and fouling. Possible synergies of volatilization ratios of other metals remain largely unclear and deserve a more systematic research.

The obtained ash is effectively utilized in the civil engineering sector as demonstrated by several research studies as it meets the technical requirements for cement and mortar additives. Ash properties are significantly altered in the co-combustion regime if the ash content in the co-combusted fuel and its mass share in the fuel blend are sufficiently high. High calorific value biomass with low ash content should thus be co-combusted preferentially.

As an established technology, co-combustion has to face the near future competition with alternative pathways of biomass and waste conversion to other products, fuels and chemicals, to contribute to long-term reduction of greenhouse gases emissions. On the other hand, the expected increase of power consumption as a result of electro mobility development is to its advantage. However, the expected gradual shutdown of coal power plants will limit the direct co-combustion potential in further decades.

Author Contributions: Conceptualization: M.V., J.K. and L.L.; methodology: M.V., A.V. and G.J.; investigation: J.J.; Data Curation: M.V. and O.M.; writing—original draft preparation: M.V. and J.K.; writing-Review and Editing: M.R. and O.M.; visualization: J.J.; funding acquisition: A.V. and M.R. All authors have read and agreed to the published version of the manuscript.

Funding: This work was financially supported by the Slovak Research and Development Agency under the contracts Nr. APVV-19-0170, APVV-16-0192 and APVV-15-0148.

Institutional Review Board Statement: Not applicable.

Informed Consent Statement: Not applicable.

Data Availability Statement: Data sharing not applicable.

Conflicts of Interest: The authors declare no conflict of interest. 


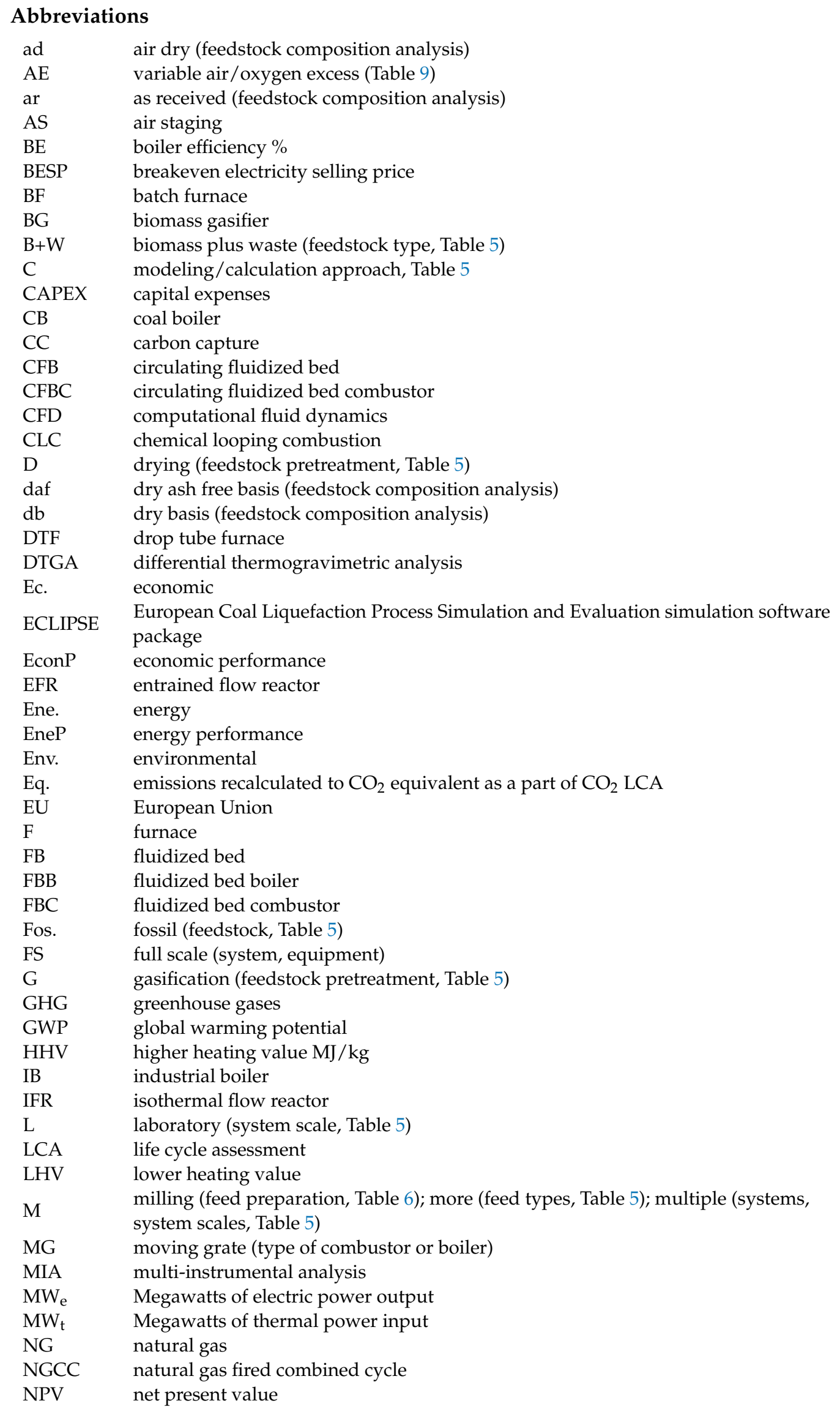




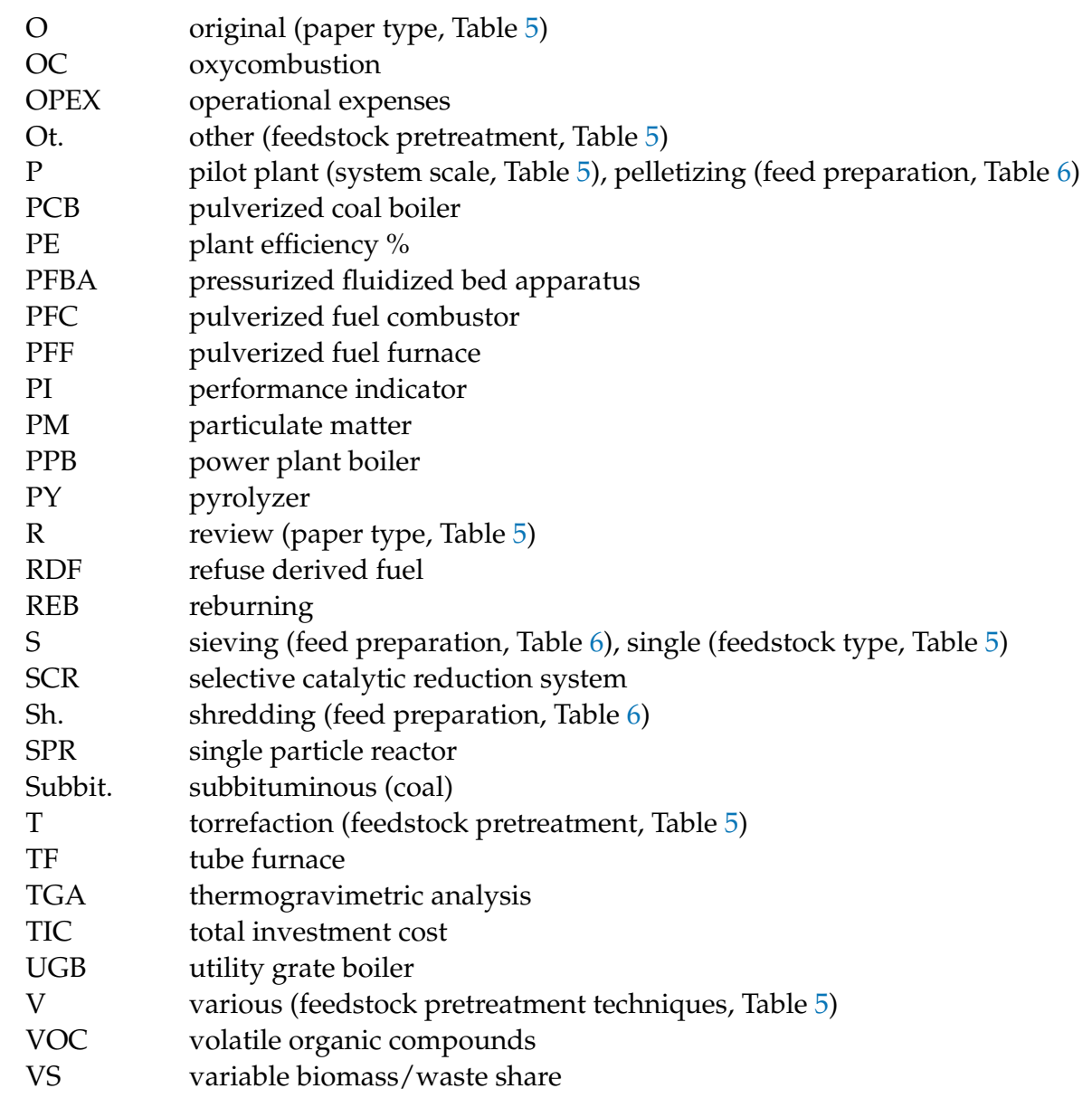

\section{References}

1. Puigjaner, L.; Pérez-Fortes, M.; Laínez-Aguirre, J. Towards a carbon-neutral energy sector: Opportunities and challenges of coordinated bioenergy supply chains-A PSE approach. Energies 2015, 8, 5613-5660. [CrossRef]

2. Rehfeldt, M.; Worrell, E.; Eichhammer, W.; Fleiter, T. A review of the emission reduction potential of fuel switch towards biomass and electricity in European basic materials industry until 2030. Renew. Sustain. Energy Rev. 2020, 120, 109672. [CrossRef]

3. Fogassy, G.; Thegarid, N.; Toussaint, G.; van Veen, A.C.; Schuurman, Y.; Mirodatos, C. Biomass derived feedstock co-processing with vacuum gas oil for second-generation fuel production in FCC units. Appl. Catal. B Environ. 2010, 96, 476-485. [CrossRef]

4. Baloch, H.A.; Nizamuddin, S.; Siddiqui, M.T.H.; Riaz, S.; Jatoi, A.S.; Dumbre, D.K.; Mubarak, N.M.; Srinivasan, M.P.; Griffin, G.J. Recent advances in production and upgrading of bio-oil from biomass: A critical overview. J. Environ. Chem. Eng. 2018, 6, 5101-5118. [CrossRef]

5. Mandal, S.; Haydary, J.; Bhattacharya, T.K.; Tanna, H.R.; Husár, J.; Ház, A. Valorization of pine needles by thermal conversion to solid, liquid and gaseous fuels in a screw reactor. Waste Biomass Valorization 2019, 10, 3587-3599. [CrossRef]

6. Gandidi, I.M.; Susila, M.D.; Mustofa, A.; Pambudi, N.A. Thermal-Catalytic cracking of real MSW into Bio-Crude Oil. J. Energy Inst. 2018, 91, 304-310. [CrossRef]

7. Xu, J.; Huang, Q.; Lv, C.; Feng, Q.; Wang, F. Carbon emissions reductions oriented dynamic equilibrium strategy using biomasscoal co-firing. Energy Policy 2018, 123, 184-197. [CrossRef]

8. Sami, M.; Annamalai, K.; Wooldridge, M. Co-firing of coal and biomass fuel blends. Prog. Energy Combust. Sci. 2001, 27, 171-214. [CrossRef]

9. Hansson, J.; Berndes, G.; Johnsson, F.; Kjärstad, J. Co-firing biomass with coal for electricity generation-An assessment of the potential in EU27. Energy Policy 2009, 37, 1444-1455. [CrossRef]

10. Karampinis, E.; Grammelis, P.; Agraniotis, M.; Violidakis, I.; Kakaras, E. Co-firing of biomass with coal in thermal power plants: Technology schemes, impacts, and future perspectives. Wiley Interdiscip. Rev. Energy Environ. 2014, 3, 384-399. [CrossRef]

11. Tchapda, A.; Pisupati, S. A review of thermal co-conversion of coal and biomass/waste. Energies 2014, 7, 1098-1148. [CrossRef]

12. Haydary, J.; Susa, D.; Dudáš, J. Pyrolysis of aseptic packages (tetrapak) in a laboratory screw type reactor and secondary thermal/catalytic tar decomposition. Waste Manag. 2013, 33, 1136-1141. [CrossRef] [PubMed]

13. Dai, J.; Sokhansanj, S.; Grace, J.R.; Bi, X.; Lim, C.J.; Melin, S. Overview and some issues related to co-firing biomass and coal. Can J. Chem. Eng. 2008, 86, 367-386. [CrossRef] 
14. Sikarwar, V.S.; Zhao, M.; Fennell, P.S.; Shah, N.; Anthony, E.J. Progress in biofuel production from gasification. Prog. Energy Combust. Sci. 2017, 61, 189-248. [CrossRef]

15. Sondreal, E.A.; Benson, S.A.; Hurley, J.P.; Mann, M.D.; Pavlish, J.H.; Swanson, M.L.; Weber, G.F.; Zygarlicke, C.J. Review of advances in combustion technology and biomass cofiring. Fuel Process. Technol. 2001, 71, 7-38. [CrossRef]

16. Lintunen, J.; Kangas, H.-L. The case of co-firing: The market level effects of subsidizing biomass co-combustion. Energy Econ. 2010, 32, 694-701. [CrossRef]

17. Hein, K.R.G.; Bemtgen, J.M. EU clean coal technology-Co-combustion of coal and biomass. Fuel Process. Technol. 1998, 54, 159-169. [CrossRef]

18. Pedersen, L.S.; Nielsen, H.P.; Kiil, S.; Hansen, L.A.; Dam-Johansen, K.; Kildsig, F.; Christensen, J.; Jespersen, P. Full-scale co-firing of straw and coal. Fuel 1996, 75, 1584-1590. [CrossRef]

19. Tillman, D.A. Biomass cofiring: The technology, the experience, the combustion consequences. Biomass Bioenergy 2000, 19, 365-384. [CrossRef]

20. Drosatos, P.; Nikolopoulos, N.; Karampinis, E.; Grammelis, P.; Kakaras, E. Comparative investigation of a co-firing scheme in a lignite-fired boiler at very low thermal-load operation using either pre-dried lignite or biomass as supporting fuel. Fuel Process. Technol. 2018, 180, 140-154. [CrossRef]

21. Sefidari, H.; Ma, C.; Fredriksson, C.; Lindblom, B.; Wiinikka, H.; Nordin, L.O.; Wu, G.; Yazhenskikh, E.; Müller, M.; Öhman, M. The effect of co-firing coal and woody biomass upon the slagging/deposition tendency in iron-ore pelletizing grate-kiln plants. Fuel Process. Technol. 2020, 199, 106254. [CrossRef]

22. Chen, G.-B.; Chatelier, S.; Lin, H.-T.; Wu, F.-H.; Lin, T.-H. A study of sewage sludge co-combustion with Australian black coal and shiitake substrate. Energies 2018, 11, 3436. [CrossRef]

23. Kazagic, A.; Hodzic, N.; Metovic, S. Co-combustion of low-rank coal with woody biomass and miscanthus: An experimental study. Energies 2018, 11, 601. [CrossRef]

24. Sh, L.; Jeong, T.-Y.; Jeon, K.-T.; Park, K.-W.; Lee, B.-H.; Jeon, C.-H. Combustion behaviors of wood pellet fuel and its co-firing with different coals. J. Mech. Sci. Technol. 2019, 33, 4545-4553. [CrossRef]

25. Liu, X.; Chen, M.; Wei, Y. Assessment on oxygen enriched air co-combustion performance of biomass/bituminous coal. Renew. Energy 2016, 92, 428-436. [CrossRef]

26. Xue, J.; Chellappa, T.; Ceylan, S.; Goldfarb, J.L. Enhancing biomass + coal Co-firing scenarios via biomass torrefaction and carbonization: Case study of avocado pit biomass and Illinois No. 6 coal. Renew. Energy 2018, 122, 152-162. [CrossRef]

27. Isaac, K.; Bada, S.O. The co-combustion performance and reaction kinetics of refuse derived fuels with South African high ash coal. Heliyon 2020, 6, e03309. [CrossRef]

28. Fuller, A.; Maier, J.; Karampinis, E.; Kalivodova, J.; Grammelis, P.; Kakaras, E.; Scheffknecht, G. Fly ash formation and characteristics from (co-)combustion of an herbaceous biomass and a Greek lignite (low-rank coal) in a pulverized fuel pilot-scale test facility. Energies 2018, 11, 1581. [CrossRef]

29. Xue, Z.; Zhong, Z.; Lai, X. Investigation on gaseous pollutants emissions during co-combustion of coal and wheat straw in a fluidized bed combustor. Chemosphere 2020, 240, 124853. [CrossRef]

30. Battista, J.J.; Hughes, E.E.; Tillman, D.A. Biomass cofiring at Seward Station. Biomass Bioenergy 2000, 19, 419-427.

31. Agbor, E.; Zhang, X.; Kumar, A. A review of biomass co-firing in North America. Renew. Sustain. Energy Rev. 2014, 40, 930-943. [CrossRef]

32. Al-Mansour, F.; Zuwala, J. An evaluation of biomass co-firing in Europe. Biomass Bioenergy 2010, 34, 620-629. [CrossRef]

33. Narayanan, K.V.; Natarajan, E. Experimental studies on cofiring of coal and biomass blends in India. Renew. Energy 2007, 32, 2548-2558. [CrossRef]

34. Tillman, D.A. Biomass cofiring for emissions management. In Handbook of Clean Energy Systems; Wiley: Hoboken, NJ, USA, 2015; pp. 1-22.

35. Baxter, L. Biomass-coal co-combustion: Opportunity for affordable renewable energy. Fuel 2005, 84, 1295-1302. [CrossRef]

36. Leckner, B. Co-combustion: A summary of technology. Therm. Sci. 2007, 11, 5-40. [CrossRef]

37. Pronobis, M. The influence of biomass co-combustion on boiler fouling and efficiency. Fuel 2006, 85, 474-480. [CrossRef]

38. Priyanto, D.E.; Ueno, S.; Sato, N.; Kasai, H.; Tanoue, T.; Fukushima, H. Ash transformation by co-firing of coal with high ratios of woody biomass and effect on slagging propensity. Fuel 2016, 174, 172-179. [CrossRef]

39. Opydo, M.; Dudek, A.; Kobyłecki, R. Characteristics of solids accumulation on steel samples during co-combustion of biomass and coal in a CFB boiler. Biomass Bioenergy 2019, 120, 291-300. [CrossRef]

40. Yao, X.; Zheng, Y.; Zhou, H.; Xu, K.; Xu, Q.; Li, L. Effects of biomass blending, ashing temperature and potassium addition on ash sintering behaviour during co-firing of pine sawdust with a Chinese anthracite. Renew. Energy 2020, 147, 2309-2320. [CrossRef]

41. Duan, L.; Duan, Y.; Zhao, C.; Anthony, E.J. NO emission during co-firing coal and biomass in an oxy-fuel circulating fluidized bed combustor. Fuel 2015, 150, 8-13. [CrossRef]

42. Varol, M.; Symonds, R.; Anthony, E.J.; Lu, D.; Jia, L.; Tan, Y. Emissions from co-firing lignite and biomass in an oxy-fired CFBC. Fuel Process. Technol. 2018, 173, 126-133. [CrossRef]

43. Zhang, Z.; Zeng, Q. Numerical simulation and experimental analysis on nitrogen and sulfur oxides emissions during the co-combustion of Longyan anthracite and sawmill sludge. Fuel 2019, 254, 115611. [CrossRef] 
44. Skodras, G.; Grammelis, P.; Samaras, P.; Vourliotis, P.; Kakaras, E.; Sakellaropoulos, G.P. Emissions monitoring during coal waste wood co-combustion in an industrial steam boiler. Fuel 2002, 81, 547-554. [CrossRef]

45. Finney, K.N.; Szuhanszki, J.; Darvell, L.I.; Dooley, B.; Milkowski, K.; Jones, J.M.; Pourkashanian, M. Entrained metal aerosol emissions from air-fired biomass and coal combustion for carbon capture applications. Materials 2018, 11, 1819. [CrossRef] [PubMed]

46. Fu, B.; Liu, G.; Mian, M.M.; Zhou, C.; Sun, M.; Wu, D.; Liu, Y. Co-combustion of industrial coal slurry and sewage sludge: Thermochemical and emission behavior of heavy metals. Chemosphere 2019, 233, 440-451. [CrossRef]

47. Zhuang, X.; Song, Y.; Zhan, H.; Yin, X.; Wu, C. Synergistic effects on the co-combustion of medicinal biowastes with coals of different ranks. Renew. Energy 2019, 140, 380-389. [CrossRef]

48. Glushkov, D.; Kuznetsov, G.; Paushkina, K. Switching coal-fired thermal power plant to composite fuel for recovering industrial and municipal waste: Combustion characteristics, emissions, and economic effect. Energies 2020, 13, 259. [CrossRef]

49. Sung, Y.; Lee, S.; Kim, C.; Jun, D.; Moon, C.; Choi, G.; Kim, D. Synergistic effect of co-firing woody biomass with coal on NOx reduction and burnout during air-staged combustion. Exp. Therm. Fluid Sci. 2016, 71, 114-125. [CrossRef]

50. Namkung, H.; Lee, Y.-J.; Park, J.-H.; Song, G.-S.; Choi, J.W.; Choi, Y.-C.; Park, S.-J.; Kim, J.-G. Blending effect of sewage sludge and woody biomass into coal on combustion and ash agglomeration behavior. Fuel 2018, 225, 266-276. [CrossRef]

51. Kalembkiewicz, J.; Chmielarz, U. Ashes from co-combustion of coal and biomass: New industrial wastes. Resour. Conserv. Recycl. 2012, 69, 109-121. [CrossRef]

52. Verma, M.; Loha, C.; Sinha, A.N.; Chatterjee, P.K. Drying of biomass for utilising in co-firing with coal and its impact on environment-A review. Renew. Sustain. Energy Rev. 2017, 71, 732-741. [CrossRef]

53. Piwowar, A.; Dzikuć, M. Outline of the economic and technical problems associated with the co-combustion of biomass in Poland. Renew. Sustain. Energy Rev. 2016, 54, 415-420. [CrossRef]

54. McIlveen-Wright, D.R.; Huang, Y.; Rezvani, S.; Mondol, J.D.; Redpath, D.; Anderson, M.; Hewitt, N.J.; Williams, B.C. A Technoeconomic assessment of the reduction of carbon dioxide emissions through the use of biomass co-combustion. Fuel 2011, 90, 11-18. [CrossRef]

55. Agbor, E.; Oyedun, A.O.; Zhang, X.; Kumar, A. Integrated techno-economic and environmental assessments of sixty scenarios for co-firing biomass with coal and natural gas. Appl. Energy 2016, 169, 433-449. [CrossRef]

56. Miedema, J.H.; Benders, R.M.J.; Moll, H.C.; Pierie, F. Renew, reduce or become more efficient? The climate contribution of biomass co-combustion in a coal-fired power plant. Appl. Energy 2017, 187, 873-885. [CrossRef]

57. Black, S.; Szuhánszki, J.; Pranzitelli, A.; Ma, L.; Stanger, P.J.; Ingham, D.B.; Pourkashanian, M. Effects of firing coal and biomass under oxy-fuel conditions in a power plant boiler using CFD modelling. Fuel 2013, 113, 780-786. [CrossRef]

58. Pérez-Jeldres, R.; Cornejo, P.; Flores, M.; Gordon, A.; García, X. A modeling approach to co-firing biomass/coal blends in pulverized coal utility boilers: Synergistic effects and emissions profiles. Energy 2017, 120, 663-674. [CrossRef]

59. Milićević, A.; Belošević, S.; Crnomarković, N.; Tomanović, I.; Tucaković, D. Mathematical modelling and optimisation of lignite and wheat straw co-combustion in 350 MWe boiler furnace. Appl. Energy 2020, 260, 114206. [CrossRef]

60. Alakangas, E.; Junginger, M.; van Dam, J.; Hinge, J.; Keränen, J.; Olsson, O.; Porsö, C.; Martikainen, A.; Rathbauer, J.; Sulzbacher, L.; et al. EUBIONET III-Solutions to biomass trade and market barriers. Renew. Sustain. Energy Rev. 2012, 16, 4277-4290. [CrossRef]

61. Mesfun, S.; Leduc, S.; Patrizio, P.; Wetterlund, E.; Mendoza-Ponce, A.; Lammens, T.; Staritsky, I.; Elbersen, B.; Lundgren, J.; Kraxner, F. Spatio-temporal assessment of integrating intermittent electricity in the EU and Western Balkans power sector under ambitious $\mathrm{CO}_{2}$ emission policies. Energy 2018, 164, 676-693. [CrossRef]

62. Brosowski, A.; Thrän, D.; Mantau, U.; Mahro, B.; Erdmann, G.; Adler, P.; Stinner, W.; Reinhold, G.; Hering, T.; Blanke, C. A review of biomass potential and current utilization-Status quo for 93 biogenic wastes and residues in Germany. Biomass Bioenergy 2016, 95, 257-272. [CrossRef]

63. López, R.; Menéndez, M.; Fernández, C.; Bernardo-Sánchez, A. The effects of scale-up and coal-biomass blending on supercritical coal oxy-combustion power plants. Energy 2018, 148, 571-584. [CrossRef]

64. Hughes, E. Biomass cofiring: Economics, policy and opportunities. Biomass Bioenergy 2000, 19, 457-465. [CrossRef]

65. Mann, M.; Spath, P. A life cycle assessment of biomass cofiring in a coal-fired power plant. Clean Prod. Process. 2001, 3, 81-91. [CrossRef]

66. Royo, J.; Sebastián, F.; García-Galindo, D.; Gómez, M.; Díaz, M. Large-scale analysis of GHG (greenhouse gas) reduction by means of biomass co-firing at country-scale: Application to the Spanish case. Energy 2012, 48, 255-267. [CrossRef]

67. Lüschen, A.; Madlener, R. Economic viability of biomass cofiring in new hard-coal power plants in Germany. Biomass Bioenergy 2013, 57, 33-47. [CrossRef]

68. Gu, H.; Zhang, K.; Wang, Y.; Huang, Y.; Hewitt, N.; Roskilly, A.P. Waste biomass from production process co-firing with coal in a steam boiler to reduce fossil fuel consumption: A case study. J. Energy Chem. 2013, 22, 413-419. [CrossRef]

69. Kazagic, A.; Music, M.; Smajevic, I.; Ademovic, A.; Redzic, E. Possibilities and sustainability of "biomass for power" solutions in the case of a coal-based power utility. Clean Technol. Environ. Policy 2016, 18, 1675-1683. [CrossRef]

70. Mohd Idris, M.N.; Hashim, H.; Razak, N.H. Spatial optimisation of oil palm biomass co-firing for emissions reduction in coal-fired power plant. J. Clean. Prod. 2018, 172, 3428-3447. [CrossRef]

71. Nyashina, G.; Strizhak, P. Impact of forest fuels on gas emissions in coal slurry fuel combustion. Energies 2018, 11, 2491. [CrossRef] 
72. Pikoń, K.; Krawczyk, P.; Badyda, K.; Bogacka, M. Predictive analysis of waste co-combustion with fossil fuels using the life cycle assessment (LCA) methodology. Energies 2019, 12, 3691. [CrossRef]

73. Smoliński, A.; Karwot, J.; Bondaruk, J.; Bak, A. The bioconversion of sewage sludge to bio-fuel: The environmental and economic benefits. Materials 2019, 12, 2417. [CrossRef] [PubMed]

74. Truong, A.H.; Patrizio, P.; Leduc, S.; Kraxner, F.; Ha-Duong, M. Reducing emissions of the fast growing Vietnamese coal sector: The chances offered by biomass co-firing. J. Clean. Prod. 2019, 215, 1301-1311. [CrossRef]

75. Qin, K.; Thunman, H. Diversity of chemical composition and combustion reactivity of various biomass fuels. Fuel 2015, 147, 161-169. [CrossRef]

76. Arromdee, P.; Kuprianov, V.I. Experimental study and empirical modeling of $\mathrm{CO}$ and NO behaviors in a fluidized-bed combustor firing pelletized biomass fuels. Biomass Convers. Biorefinery 2020. [CrossRef]

77. Razmjoo, N.; Sefidari, H.; Strand, M. Measurements of temperature and gas composition within the burning bed of wet woody residues in a 4 MW moving grate boiler. Fuel Process. Technol. 2016, 152, 438-445. [CrossRef]

78. Junga, R.; Knauer, W.; Niemiec, P.; Tańczuk, M. Experimental tests of co-combustion of laying hens manure with coal by using thermogravimetric analysis. Renew. Energy 2017, 111, 245-255. [CrossRef]

79. Haydary, J.; Susa, D.; Gelinger, V.; Čacho, F. Pyrolysis of automobile shredder residue in a laboratory scale screw type reactor. J. Environ. Chem. Eng. 2016, 4, 965-972. [CrossRef]

80. Haydary, J.; Susa, D. Kinetics of thermal decomposition of aseptic packages. Chem. Pap. 2013, 67, 1514-1520. [CrossRef]

81. Murphy, J.J.; Shaddix, C.R. Combustion kinetics of coal chars in oxygen-enriched environments. Combust. Flame 2006, 144, 710-729. [CrossRef]

82. Svoboda, K.; Pohořelý, M.; Hartman, M. Effects of operating conditions and dusty fuel on the $\mathrm{NOx}, \mathrm{N}_{2} \mathrm{O}$, and CO emissions in PFB co-combustion of coal and wood. Energy Fuels 2003, 17, 1091-1099. [CrossRef]

83. Cong, K.; Han, F.; Zhang, Y.; Li, Q. The investigation of co-combustion characteristics of tobacco stalk and low rank coal using a macro-TGA. Fuel 2019, 237, 126-132. [CrossRef]

84. Ohlemüller, P.; Ströhle, J.; Epple, B. Chemical looping combustion of hard coal and torrefied biomass in a 1 MW th pilot plant. Int. J. Greenh. Gas Control 2017, 65, 149-159. [CrossRef]

85. Atimtay, A.T.; Kayahan, U.; Unlu, A.; Engin, B.; Varol, M.; Olgun, H.; Atakul, H. Co-firing of pine chips with Turkish lignites in $750 \mathrm{kWth}$ circulating fluidized bed combustion system. Bioresour. Technol. 2017, 224, 601-610. [CrossRef] [PubMed]

86. Pawlak-Kruczek, H.; Ostrycharczyk, M.; Czerep, M.; Baranowski, M.; Zgóra, J. Examinations of the process of hard coal and biomass blend combustion in OEA (oxygen enriched atmosphere). Energy 2015, 92, 40-46. [CrossRef]

87. Lawrence, A.; Kumar, R.; Nandakumar, K.; Narayanan, K. A Novel tool for assessing slagging propensity of coals in PF boilers. Fuel 2008, 87, 946-950. [CrossRef]

88. Sajdak, M.; Kmieć, M.; Micek, B.; Hrabak, J. Determination of the optimal ratio of coal to biomass in the co-firing process: Feed mixture properties. Int. J. Environ. Sci. Technol. 2019, 16, 2989-3000. [CrossRef]

89. Demirbaş, A. Sustainable cofiring of biomass with coal. Energy Convers. Manag. 2003, 44, 1465-1479. [CrossRef]

90. Yao, X.; Zhou, H.; Xu, K.; Xu, Q.; Li, L. Evaluation of the fusion and agglomeration properties of ashes from combustion of biomass, coal and their mixtures and the effects of $\mathrm{K}_{2} \mathrm{CO}_{3}$ additives. Fuel 2019, 255, 115829. [CrossRef]

91. Yanik, J.; Duman, G.; Karlstrom, O.; Brink, A. NO and $\mathrm{SO}_{2}$ emissions from combustion of raw and torrefied biomasses and their blends with lignite. J. Environ. Manag. 2018, 227, 155-161. [CrossRef]

92. Xu, Z.; Zinchik, S.; Kolapkar, S.S.; Bar-Ziv, E.; Hansen, T.; Conn, D.; McDonald, A.G. Properties of torrefied U.S. waste blends. Front. Energy Res. 2018, 6, 65. [CrossRef]

93. Mak, J.; Landry, H.; Grieger, L.; Agnew, J.; Krigstin, S.; Helmeste, C.; Wetzel, S.; Madrali, S.; Volpé, S. An assessment of ambient and heated forced air drying pre-treatments for enhancing the quality of various forest biomass feedstocks. Front. Energy Res. 2020, 8, 7. [CrossRef]

94. Brammer, J.G.; Bridgwater, A.V. Drying technologies for an integrated gasification bio-energy plant. Renew. Sustain. Energy Rev. 1999, 3, 243-289. [CrossRef]

95. Carapellucci, R. Power generation using dedicated woody crops: Thermodynamics and economics of integrated plants. Renew. Energy 2002, 27, 143-159. [CrossRef]

96. Nyashina, G.S.; Kurgankina, M.A.; Strizhak, P.A. Environmental, economic and energetic benefits of using coal and oil processing waste instead of coal to produce the same amount of energy. Energy Convers. Manag. 2018, 174, 175-187. [CrossRef]

97. Wilk, M.; Magdziarz, A.; Kalemba, I. Characterisation of renewable fuels' torrefaction process with different instrumental techniques. Energy 2015, 87, 259-269. [CrossRef]

98. Niu, Y.; Lv, Y.; Lei, Y.; Liu, S.; Liang, Y.; Wang, D.; Hui, S.E. Biomass torrefaction: Properties, applications, challenges, and economy. Renew. Sustain. Energy Rev. 2019, 115, 109395. [CrossRef]

99. Chen, Y.; Liu, B.; Yang, H.; Yang, Q.; Chen, H. Evolution of functional groups and pore structure during cotton and corn stalks torrefaction and its correlation with hydrophobicity. Fuel 2014, 137, 41-49. [CrossRef]

100. Chen, W.-H.; Lu, K.-M.; Tsai, C.-M. An experimental analysis on property and structure variations of agricultural wastes undergoing torrefaction. Appl. Energy 2012, 100, 318-325. [CrossRef]

101. Mamvura, T.A.; Danha, G. Biomass torrefaction as an emerging technology to aid in energy production. Heliyon 2020, 6, e03531. [CrossRef] [PubMed] 
102. Tumuluru, J.S. Comparison of chemical composition and energy property of torrefied switchgrass and corn stover. Front. Energy Res. 2015, 3, 46. [CrossRef]

103. Madanayake, B.N.; Gan, S.; Eastwick, C.; Ng, H.K. Biomass as an energy source in coal co-firing and its feasibility enhancement via pre-treatment techniques. Fuel Process. Technol. 2017, 159, 287-305. [CrossRef]

104. Harding, N.S.; Adams, B.R. Biomass as a reburning fuel: A specialized cofiring application. Biomass Bioenergy 2000, 19, 429-445. [CrossRef]

105. Wieck-Hansen, K.; Overgaard, P.; Larsen, O.H. Cofiring coal and straw in a 150 MWe power boiler experiences. Biomass Bioenergy 2000, 19, 395-409. [CrossRef]

106. Savolainen, K. Co-firing of biomass in coal-fired utility boilers. Appl. Energy 2003, 74, 369-381. [CrossRef]

107. Backreedy, R.I.; Fletcher, L.M.; Jones, J.M.; Ma, L.; Pourkashanian, M.; Williams, A. Co-firing pulverised coal and biomass: A modeling approach. Proc. Combust. Inst. 2005, 30, 2955-2964. [CrossRef]

108. Nevalainen, H.; Jegoroff, M.; Saastamoinen, J.; Tourunen, A.; Jäntti, T.; Kettunen, A.; Johnsson, F.; Niklasson, F. Firing of coal and biomass and their mixtures in $50 \mathrm{~kW}$ and $12 \mathrm{MW}$ circulating fluidized beds-Phenomenon study and comparison of scales. Fuel 2007, 86, 2043-2051. [CrossRef]

109. Arias, B.; Pevida, C.; Rubiera, F.; Pis, J.J. Effect of biomass blending on coal ignition and burnout during oxy-fuel combustion. Fuel 2008, 87, 2753-2759. [CrossRef]

110. Hu, X.; Dong, C.; Yang, Y.; Zhang, J. The effect of biomass pyrolysis gas reburning on $\mathrm{N}_{2} \mathrm{O}$ emission in a coal-fired fluidized bed boiler. Chin. Sci. Bull. 2011, 56, 1429-1433. [CrossRef]

111. Casaca, C.; Costa, M. Detailed measurements in a laboratory furnace with reburning. Fuel 2011, 90, 1090-1100. [CrossRef]

112. Werle, S. A reburning process using sewage sludge-derived syngas. Chem. Pap. 2012, 66, 99-107. [CrossRef]

113. Bragato, M.; Joshi, K.; Carlson, J.B.; Tenório, J.A.S.; Levendis, Y.A. Combustion of coal, bagasse and blends thereof. Fuel 2012, 96, 43-50. [CrossRef]

114. Mehmood, S.; Reddy, B.V.; Rosen, M.A. Energy analysis of a biomass co-firing based pulverized coal power generation system. Sustainability 2012, 4, 462-490. [CrossRef]

115. Díaz-Ramírez, M.; Sebastián, F.; Royo, J.; Rezeau, A. Combustion requirements for conversion of ash-rich novel energy crops in a $250 \mathrm{kWth}$ multifuel grate fired system. Energy 2012, 46, 636-643. [CrossRef]

116. Riaza, J.; Gil, M.V.; Âlvarez, L.; Pevida, C.; Pis, J.J.; Rubiera, F. Oxy-fuel combustion of coal and biomass blends. Energy 2012, 41, 429-435. [CrossRef]

117. Moon, C.; Sung, Y.; Ahn, S.; Kim, T.; Choi, G.; Kim, D. Effect of blending ratio on combustion performance in blends of biomass and coals of different ranks. Exp. Therm. Fluid Sci. 2013, 47, 232-240. [CrossRef]

118. Haykiri-Acma, H.; Yaman, S.; Kucukbayrak, S. Co-combustion of low rank coal/waste biomass blends using dry air or oxygen. Appl. Therm. Eng. 2013, 50, 251-259. [CrossRef]

119. Hrycko, P.; Lasek, J.; Matuszek, K. Biomass gasification and Polish coal-fired boilers for process of reburning in small boilers. J. Cent. South Univ. 2013, 20, 1623-1630.

120. Steer, J.; Marsh, R.; Griffiths, A.; Malmgren, A.; Riley, G. Biomass co-firing trials on a down-fired utility boiler. Energy Convers. Manag. 2013, 66, 285-294. [CrossRef]

121. Wang, X.; Hu, Z.; Deng, S.; Xiong, Y.; Tan, H. Effect of biomass/coal co-firing and air staging on NOx emission and combustion efficiency in a drop tube furnace. Energy Procedia 2014, 61, 2331-2334. [CrossRef]

122. Varol, M.; Atimtay, A.T. Effect of biomass-sulfur interaction on ash composition and agglomeration for the co-combustion of high-sulfur lignite coals and olive cake in a circulating fluidized bed combustor. Bioresour. Technol. 2015, 198, 325-331. [CrossRef] [PubMed]

123. Bhuiyan, A.A.; Naser, J. CFD modelling of co-firing of biomass with coal under oxy-fuel combustion in a large scale power plant. Fuel 2015, 159, 150-168. [CrossRef]

124. Bhuiyan, A.A.; Naser, J. Computational modelling of co-firing of biomass with coal under oxy-fuel condition in a small scale furnace. Fuel 2015, 143, 455-466. [CrossRef]

125. Chae, T.; Lee, J.; Yang, W.; Ryu, C. Characteristics of syngas reburning in a natural gas firing furnace-Effects of combustible gas species in the syngas. J. Mech. Sci. Technol. 2016, 30, 3861-3868. [CrossRef]

126. Hodžić, N.; Kazagić, A.; Smajević, I. Influence of multiple air staging and reburning on NOx emissions during co-firing of low rank brown coal with woody biomass and natural gas. Appl. Energy 2016, 168, 38-47. [CrossRef]

127. Kopczyński, M.; Lasek, J.A.; Iluk, A.; Zuwała, J. The co-combustion of hard coal with raw and torrefied biomasses (willow (Salix viminalis), olive oil residue and waste wood from furniture manufacturing). Energy 2017, 140, 1316-1325. [CrossRef]

128. Stephan, A.; Wolf, C.; Fendt, S.; Spliethoff, H. Online corrosion measurements in small- and mid-scale during pulverised biomass/coal co-combustion. Energy Procedia 2017, 120, 309-316. [CrossRef]

129. Fogarasi, S.; Cormos, C.-C. Assessment of coal and sawdust co-firing power generation under oxy-combustion conditions with carbon capture and storage. J. Clean. Prod. 2017, 142, 3527-3535. [CrossRef]

130. Kazulis, V.; Vigants, H.; Veidenbergs, I.; Blumberga, D. Biomass and natural gas co-firing—Evaluation of GHG emissions. Energy Procedia 2018, 147, 558-565. [CrossRef]

131. Panahi, A.; Tarakcioglu, M.; Schiemann, M.; Delichatsios, M.; Levendis, Y.A. On the particle sizing of torrefied biomass for co-firing with pulverized coal. Combust. Flame 2018, 194, 72-84. [CrossRef] 
132. Rokni, E.; Ren, X.; Panahi, A.; Levendis, Y.A. Emissions of $\mathrm{SO}_{2}, \mathrm{NOx}, \mathrm{CO}_{2}$, and $\mathrm{HCl}$ from Co-firing of coals with raw and torrefied biomass fuels. Fuel 2018, 211, 363-374. [CrossRef]

133. Ślefarski, R.; Jójka, J.; Czyżewski, P.; Grzymisławski, P. Experimental investigation on syngas reburning process in a gaseous fuel firing semi-idustrial combustion chamber. Fuel 2018, 217, 490-498. [CrossRef]

134. Chen, X.; Xie, J.; Mei, S.; He, F. NOx and $\mathrm{SO}_{2}$ Emissions during co-combustion of RDF and anthracite in the environment of precalciner. Energies 2018, 11, 337. [CrossRef]

135. Skopec, P.; Hrdlička, F.; Jitka, J. Co-firing of coal with a solid recovered fuel as a route of reduction of sulfur dioxide emissions. Waste Forum 2018, 1, 26-34.

136. Choi, M.; Li, X.; Kim, K.; Sung, Y.; Choi, G. Detailed in-furnace measurements in a pulverized coal-fired furnace with combined woody biomass co-firing and air staging. J. Mech. Sci. Technol. 2018, 32, 4517-4527. [CrossRef]

137. Mätzing, H.; Gehrmann, H.-J.; Seifert, H.; Stapf, D. Modelling grate combustion of biomass and low rank fuels with CFD application. Waste Manag. 2018, 78, 686-697. [CrossRef] [PubMed]

138. Guo, F.; Zhong, Z. Optimization of the co-combustion of coal and composite biomass pellets. J. Clean. Prod. 2018, 185, 399-407. [CrossRef]

139. Wang, X.; Hu, Z.; Wang, G.; Luo, X.; Ruan, R.; Jin, Q.; Tan, H. Influence of coal co-firing on the particulate matter formation during pulverized biomass combustion. J. Energy Inst. 2019, 92, 450-458. [CrossRef]

140. Bhui, B.; Vairakannu, P. Prospects and issues of integration of co-combustion of solid fuels (coal and biomass) in chemical looping technology. J. Environ. Manag. 2019, 231, 1241-1256. [CrossRef] [PubMed]

141. Buyukada, M. Investigation of thermal conversion characteristics and performance evaluation of co-combustion of pine sawdust and lignite coal using TGA, artificial neural network modeling and likelihood method. Bioresour. Technol. 2019, 287, 121461. [CrossRef]

142. Liu, Q.; Shi, Y.; Zhong, W.; Yu, A. Co-firing of coal and biomass in oxy-fuel fluidized bed for $\mathrm{CO}_{2}$ capture: A review of recent advances. Chin. J. Chem. Eng. 2019, 27, 2261-2272. [CrossRef]

143. Oladejo, J.; Shi, K.; Meng, Y.; Adegbite, S.; Wu, T. Biomass constituents' interactions with coal during co-firing. Energy Procedia 2019, 158, 1640-1645. [CrossRef]

144. Zhang, J.; Zhou, H. Investigation of the slagging characteristics during co-combustion of Shenhua coal and corn stalk: Effect of deposition surface. Fuel 2019, 256, 115939. [CrossRef]

145. Stam, A.F.; Brem, G. Fouling in coal-fired boilers: Biomass co-firing, full conversion and use of additives-A thermodynamic approach. Fuel 2019, 239, 1274-1283. [CrossRef]

146. Smith, J.D.; Suo-Antilla, A.; Sreedharan, V. Transient LES based CFD modeling of coal-biomass co-firing combustion. Fuel Process. Technol. 2019, 193, 187-196. [CrossRef]

147. Jeong, T.-Y.; Sh, L.; Kim, J.-H.; Lee, B.-H.; Jeon, C.-H. Experimental investigation of ash deposit behavior during co-combustion of bituminous coal with wood pellets and empty fruit bunches. Energies 2019, 12, 2087. [CrossRef]

148. Coimbra, R.N.; Escapa, C.; Otero, M. Comparative thermogravimetric assessment on the combustion of coal, microalgae biomass and their blend. Energies 2019, 12, 2962. [CrossRef]

149. Alobaid, F.; Busch, J.-P.; Stroh, A.; Ströhle, J.; Epple, B. Experimental measurements for torrefied biomass Co-combustion in a 1 MWth pulverized coal-fired furnace. J. Energy Inst. 2020, 93, 833-846. [CrossRef]

150. Zhang, N.; Wang, G.; Zhang, J.; Ning, X.; Li, Y.; Liang, W.; Wang, C. Study on co-combustion characteristics of hydrochar and anthracite coal. J. Energy Inst. 2020, 93, 1125-1137. [CrossRef]

151. Zhou, A.; Xu, H.; Xu, M.; Yu, W.; Li, Z.; Yang, W. Numerical investigation of biomass co-combustion with methane for NOx reduction. Energy 2020, 194, 116868. [CrossRef]

152. Zhou, L.; Deshpande, K.; Zhang, X.; Agarwal, R.K. Process simulation of chemical looping combustion using ASPEN plus for a mixture of biomass and coal with various oxygen carriers. Energy 2020, 195, 116955. [CrossRef]

153. Wang, X.; Zhang, J.; Xu, X.; Mikulčić, H.; Li, Y.; Zhou, Y.; Tan, H. Numerical study of biomass Co-firing under Oxy-MILD mode. Renew. Energy 2020, 146, 2566-2576. [CrossRef]

154. Atimtay, A.; Yurdakul, S. Combustion and Co-Combustion characteristics of torrefied poultry litter with lignite. Renew. Energy 2020, 148, 1292-1301. [CrossRef]

155. Yelverton, T.L.B.; Brashear, A.T.; Nash, D.G.; Brown, J.E.; Singer, C.F.; Kariher, P.H.; Ryan, J.V.; Burnette, P. Characterization of emissions from a pilot-scale combustor operating on coal blended with woody biomass. Fuel 2020, 264, 116774. [CrossRef]

156. Torres, M.; Portugau, P.; Castiglioni, J.; Cuña, A.; Yermán, L. Co-combustion behaviours of a low calorific Uruguayan Oil Shale with biomass wastes. Fuel 2020, 266, 117118. [CrossRef]

157. Ndou, N.R.; Bada, S.O.; Falcon, R.M.S.; Weiersbye, I.M. Co-combustion of Searsia lancea and Tamarix usneoides with high ash coal. Fuel 2020, 267, 117282. [CrossRef]

158. Tabakaev, R.; Ibraeva, K.; Kan, V.; Dubinin, Y.; Rudmin, M.; Yazykov, N.; Zavorin, A. The effect of co-combustion of waste from flour milling and highly mineralized peat on sintering of the ash residue. Energy 2020, 196, 117157. [CrossRef]

159. Šuhaj, P.; Haydary, J.; Husár, J.; Steltenpohl, P.; Šupa, I. Catalytic gasification of refuse-derived fuel in a two-stage laboratory scale pyrolysis/gasification unit with catalyst based on clay minerals. Waste Manag. 2019, 85, 1-10. [CrossRef]

160. Martínez, J.D.; Veses, A.; Mastral, A.M.; Murillo, R.; Navarro, M.V.; Puy, N.; Artigues, A.; Bartrolí, J.; García, T. Co-pyrolysis of biomass with waste tyres: Upgrading of liquid bio-fuel. Fuel Process. Technol. 2014, 119, 263-271. [CrossRef]

161. Kuba, M.; Hofbauer, H. Experimental parametric study on product gas and tar composition in dual fluid bed gasification of woody biomass. Biomass Bioenergy 2018, 115, 35-44. [CrossRef] 
162. Thunman, H.; Seemann, M.; Berdugo Vilches, T.; Maric, J.; Pallares, D.; Ström, H.; Berndes, G.; Knutsson, P.; Larsson, A.; Breitholtz, C.; et al. Advanced biofuel production via gasification-Lessons learned from 200 man-years of research activity with Chalmers' research gasifier and the GoBiGas demonstration plant. Energy Sci. Eng. 2018, 6, 6-34. [CrossRef]

163. Strezov, V.; Evans, T.J.; Hayman, C. Thermal conversion of elephant grass (Pennisetum purpureum Schum) to bio-gas, bio-oil and charcoal. Bioresour. Technol. 2008, 99, 8394-8399. [CrossRef]

164. Carmo-Calado, L.; Hermoso-Orzáez, M.J.; Mota-Panizio, R.; Guilherme-Garcia, B.; Brito, P. Co-combustion of waste tires and plastic-rubber wastes with biomass technical and environmental analysis. Sustainability 2020, 12, 1036. [CrossRef]

165. Kočanová, S.; Lukáč, L. The impact of the composition of the wood gas to emissions after combustion of wood gas. Holist. Approach Environ. 2014, 4, 111-117.

166. Motta, I.L.; Miranda, N.T.; Maciel Filho, R.; Wolf Maciel, M.R. Biomass gasification in fluidized beds: A review of biomass moisture content and operating pressure effects. Renew. Sustain. Energy Rev. 2018, 94, 998-1023. [CrossRef]

167. Pang, S. Advances in thermochemical conversion of woody biomass to energy, fuels and chemicals. Biotechnol. Adv. 2019, 37, 589-597. [CrossRef]

168. Bridgwater, A.V. Review of fast pyrolysis of biomass and product upgrading. Biomass Bioenergy 2012, 38, 68-94. [CrossRef]

169. Roy, P.; Dias, G. Prospects for pyrolysis technologies in the bioenergy sector: A review. Renew. Sustain. Energy Rev. 2017, 77, 59-69. [CrossRef]

170. Bridgwater, A.V.; Toft, A.J.; Brammer, J.G. A techno-economic comparison of power production by biomass fast pyrolysis with gasification and combustion. Renew. Sustain. Energy Rev. 2002, 6, 181-246. [CrossRef]

171. Situmorang, Y.A.; Zhao, Z.; Yoshida, A.; Abudula, A.; Guan, G. Small-scale biomass gasification systems for power generation (<200 kW class): A review. Renew. Sustain. Energy Rev. 2020, 117, 109486. [CrossRef]

172. Richardson, Y.; Blin, J.; Julbe, A. A short overview on purification and conditioning of syngas produced by biomass gasification: Catalytic strategies, process intensification and new concepts. Prog. Energy Combust. Sci. 2012, 38, 765-781. [CrossRef]

173. Smoot, L.D.; Hill, S.C.; Xu, H. NOx control through reburning. Prog. Energy Combust. Sci. 1998, 24, 385-408. [CrossRef]

174. Vainio, E.; Kinnunen, H.; Laurén, T.; Brink, A.; Yrjas, P.; DeMartini, N.; Hupa, M. Low-temperature corrosion in co-combustion of biomass and solid recovered fuels. Fuel 2016, 184, 957-965. [CrossRef]

175. Vassilev, S.V.; Vassileva, C.G.; Baxter, D. Trace element concentrations and associations in some biomass ashes. Fuel 2014, 129, 292-313. [CrossRef]

176. Vassilev, S.V.; Baxter, D.; Vassileva, C.G. An overview of the behaviour of biomass during combustion: Part I. Phase-mineral transformations of organic and inorganic matter. Fuel 2013, 112, 391-449. [CrossRef]

177. Wang, S.; Miller, A.; Llamazos, E.; Fonseca, F.; Baxter, L. Biomass fly ash in concrete: Mixture proportioning and mechanical properties. Fuel 2008, 87, 365-371. [CrossRef]

178. Vassilev, S.V.; Baxter, D.; Vassileva, C.G. An overview of the behaviour of biomass during combustion: Part II. Ash fusion and ash formation mechanisms of biomass types. Fuel 2014, 117, 152-183. [CrossRef]

179. Sarabèr, A.J. Co-combustion and its impact on fly ash quality; full-scale experiments. Fuel Process. Technol. 2014, 128, 68-82. [CrossRef]

180. Lazić, L.; Lukáč, L.; Lukáč, P.; Hršak, D. Influence of the external recirculation of flue gases on reduction of NOx at propane-butane combustion. J. Int. Sci. Publ. Ecol. Saf. 2011, 5, 4-16.

181. Villar, A.; Arribas, J.J.; Parrondo, J. Waste-to-energy technologies in continuous process industries. Clean Technol. Environ. Policy 2012, 14, 29-39. [CrossRef]

182. Haraldsson, J.; Johansson, M.T. Review of measures for improved energy efficiency in production-related processes in the aluminium industry-From electrolysis to recycling. Renew. Sustain. Energy Rev. 2018, 93, 525-548. [CrossRef]

183. Liu, G.; Bangs, C.E.; Muller, D.B. Unearthing potentials for decarbonizing the U.S. aluminum cycle. Environ. Sci. Technol. 2011, 45, 9515-9522. [CrossRef] [PubMed]

184. Dzurňák, R.; Varga, A.; Kizek, J.; Jablonský, G.; Lukáč, L. Influence of burner nozzle parameters analysis on the aluminium melting process. Appl. Sci. 2019, 9, 1614. [CrossRef]

185. Lukáč, L.; Kizek, J.; Varga, A.; Lazić, L.; Dzurňák, R. Selected parameters that influence emission formation during aluminum melting. In Coexistence of Material Science and Sustainable Technology in Economic Growth, Proceedings of the 18th International Foundrymen Conference, Sisak, Croatia, 15-17 May 2019; University of Zagreb: Zagreb, Croatia, 2019; pp. 288-294. ISBN 978-953-7082-34-5.

186. Brough, D.; Jouhara, H. The aluminium industry: A review on state-of-the-art technologies, environmental impacts and possibilities for waste heat recovery. Int. J. Thermofluids 2020, 1-2, 100007. [CrossRef]

187. Royo, P.; Ferreira, V.J.; López-Sabirón, A.M.; García-Armingol, T.; Ferreira, G. Retrofitting strategies for improving the energy and environmental efficiency in industrial furnaces: A case study in the aluminium sector. Renew. Sustain. Energy Rev. 2018, 82, 1813-1822. [CrossRef]

188. Lukáč, L.; Kizek, J.; Jablonský, G.; Karakash, Y. Defining the mathematical dependencies of NOx and CO emission generation after biomass combustion in low-power boiler. Civ. Environ. Eng. Rep. 2019, 29, 153-163. [CrossRef]

189. Lundin, L.; Gomez-Rico, M.F.; Forsberg, C.; Nordenskjold, C.; Jansson, S. Reduction of PCDD, PCDF and PCB during cocombustion of biomass with waste products from pulp and paper industry. Chemosphere 2013, 91, 797-801. [CrossRef]

190. Křůmal, K.; Mikuška, P.; Horák, J.; Hopan, F.; Krpec, K. Comparison of emissions of gaseous and particulate pollutants from the combustion of biomass and coal in modern and old-type boilers used for residential heating in the Czech Republic, Central Europe. Chemosphere 2019, 229, 51-59. [CrossRef] 